Aus der Poliklinik für Kieferorthopädie

(Prof. Dr. med. dent. P. Meyer-Marcotty)

im Zentrum Zahn-, Mund- und Kieferheilkunde

der Medizinischen Fakultät der Universität Göttingen

\title{
Messungen zur mechanischen Stabilität \\ der Gleichgewichte von Knieendoprothesen: \\ AEQUOS versus Mebio Scan-Knie
}

\author{
INAUGURAL-DISSERTATION \\ zur Erlangung des Doktorgrades \\ für Zahnheilkunde \\ der Medizinischen Fakultät der \\ vorgelegt von \\ Anja Saskia Albrecht \\ aus \\ Köln
}

Georg-August-Universität zu Göttingen

Göttingen 2019 
Dekan:

Referent/in:

Ko-Referent/in:

Drittreferent/in:
Prof. Dr. rer. nat. H. K. Kroemer

Prof. Dr. med. habil. Dr. rer. nat. H. Nägerl, Dipl.Phys.

Datum der mündlichen Prüfung: 
Hiermit erkläre ich, die Dissertation mit dem Titel "Messungen zur mechanischen Stabilität der Gleichgewichte von Knieendoprothesen: AEQUOS versus Mebio Scan-Knie" eigenständig angefertigt und keine anderen als die von mir angegebenen Quellen und Hilfsmittel verwendet zu haben.

Göttingen, den 


\section{Inhaltsverzeichnis}

Abbildungsverzeichnis ..........................................................................................................IV

Tabellenverzeichnis............................................................................................................... VII

Abkürzungsverzeichnis ...............................................................................................VIII

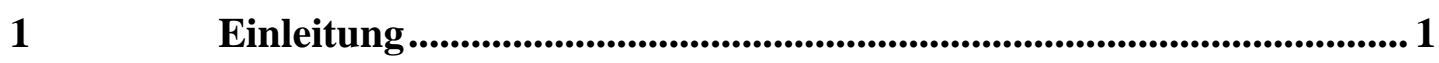

$2 \quad$ Anatomie und Biomechanik des Kniegelenks ....................................... 3

2.1 Anatomie des Tibiofemoralgelenks ............................................. 3

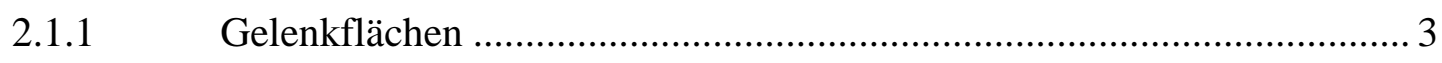

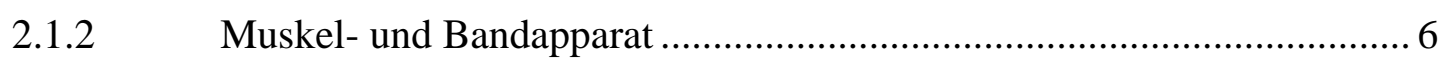

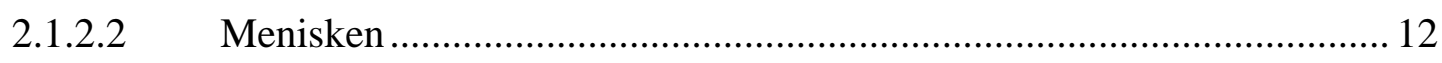

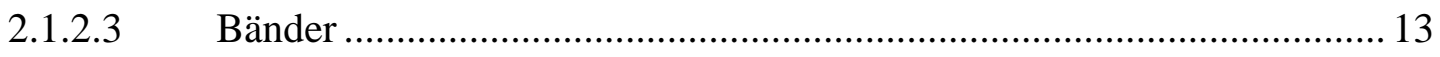

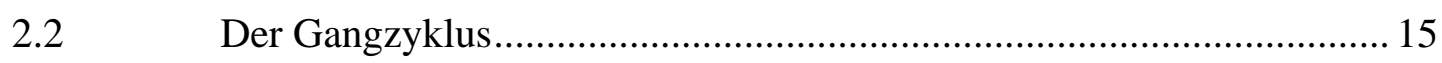

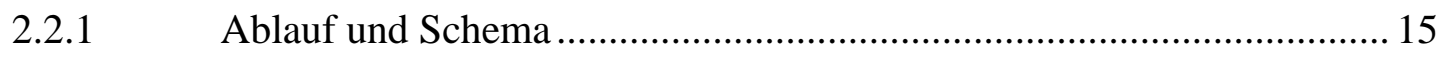

2.2.2 Aufgabe des Kniegelenks im Gangzyklus …................................... 17

2.3 Biomechanik des Tibiofemoralgelenks ........................................... 18

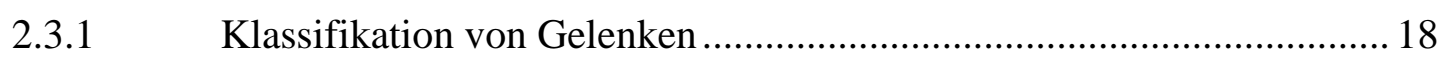

2.3.2 Funktionsmodell des Kniegelenks ................................................ 19

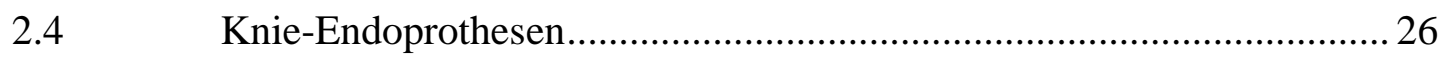

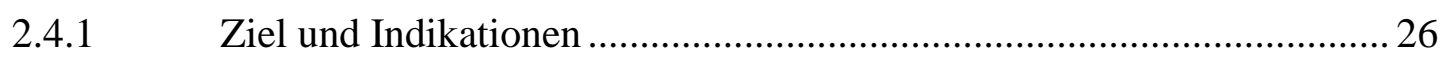

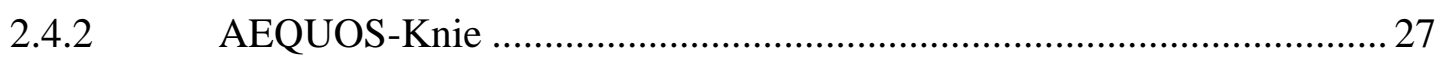

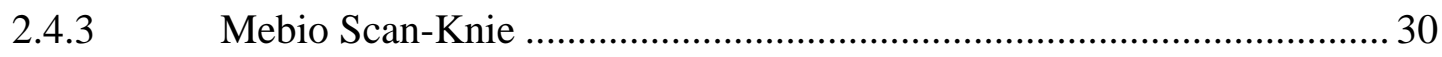

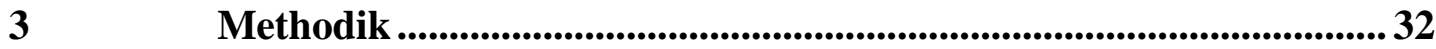

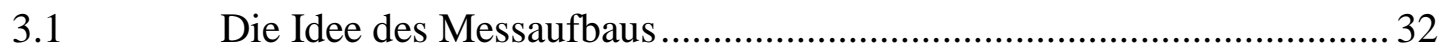

3.1.1 Entwicklung und Prinzip des Messaufbaus.......................................... 34

3.1.2 Anforderungen an die Konzeption ................................................... 37

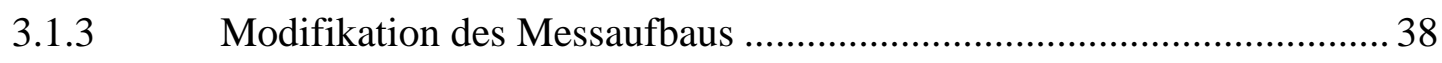

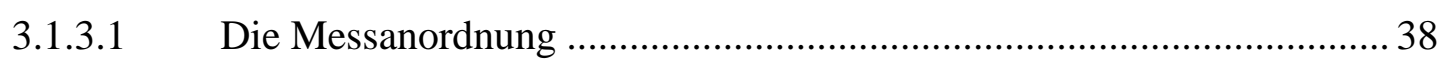

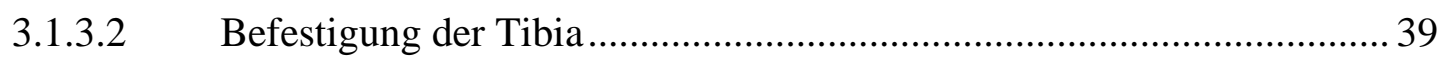


3.1.3.3 Gewichte zur Erzeugung der ,Muskelkräfte“ ...................................... 40

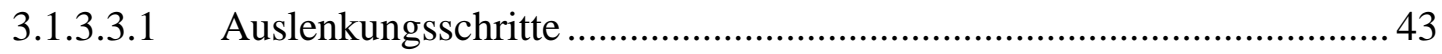

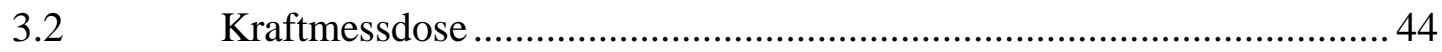

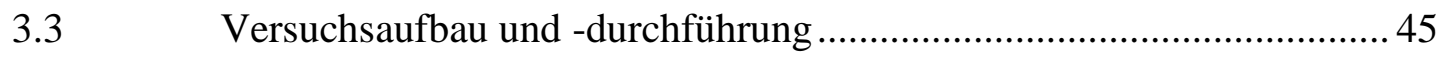

3.3.1 Fixierung des Untersuchungsmaterials ........................................... 46

3.3.2 Herstellung von Gleichgewichtslagen........................................... 48

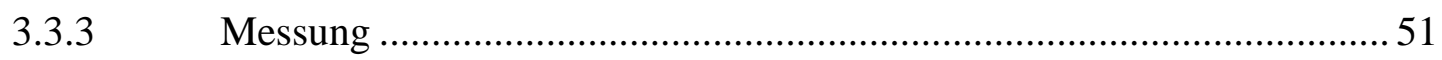

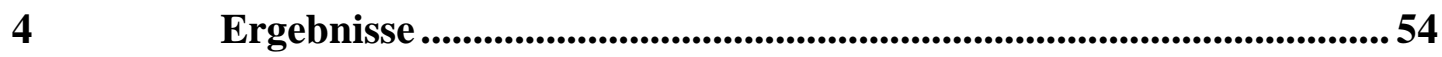

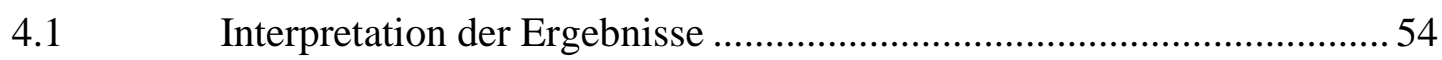

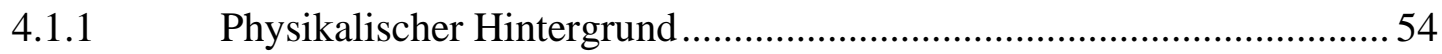

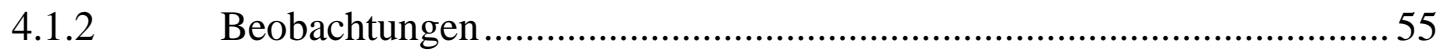

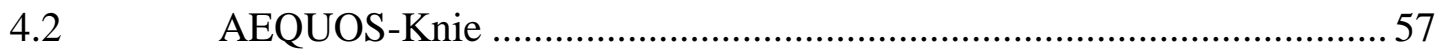

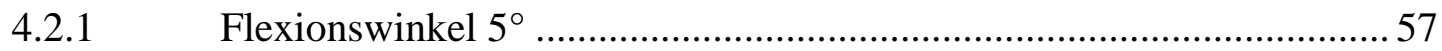

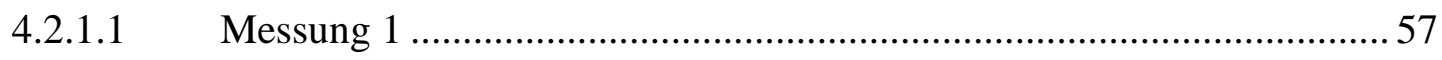

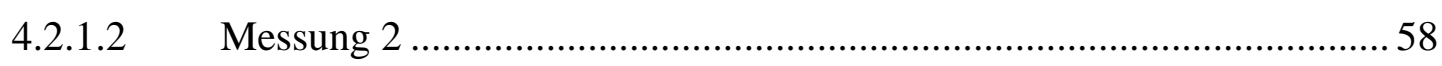

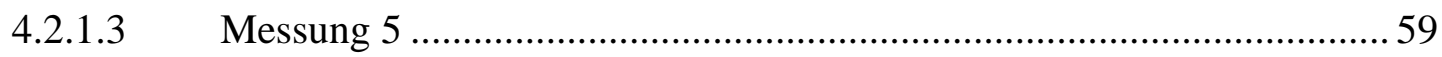

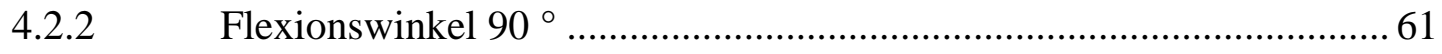

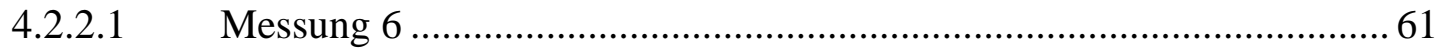

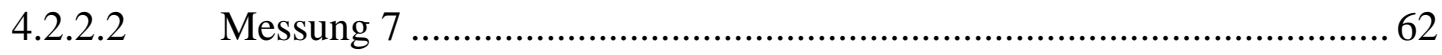

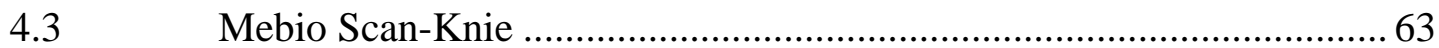

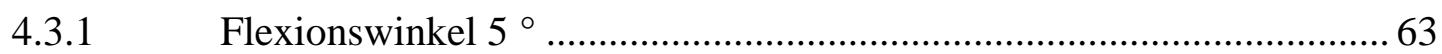

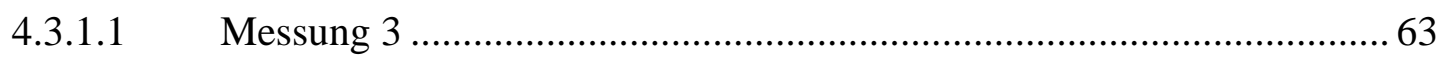

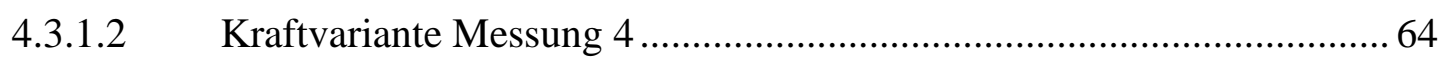

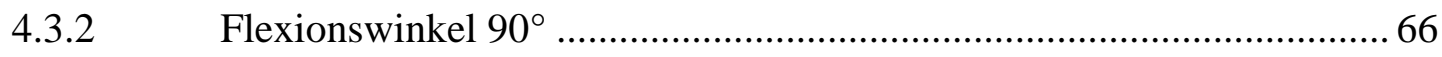

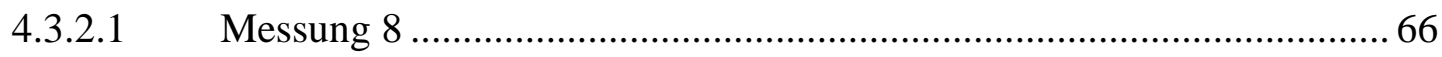

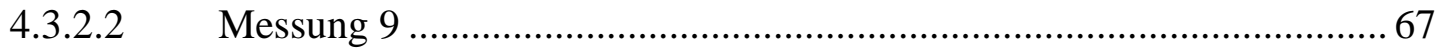

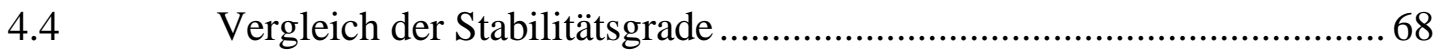

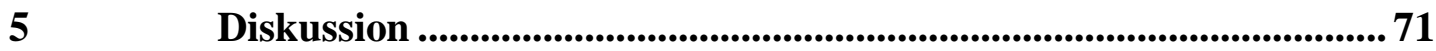

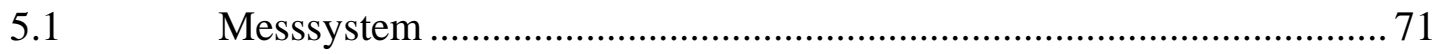

5.2 Muskelkräfte und Flexionswinkel................................................... 72

5.3 Vergleich von AEQUOS-Knie und Mebio Scan-Knie ......................... 74 


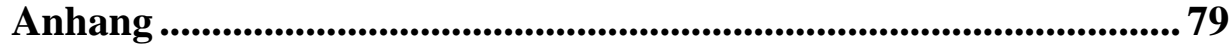

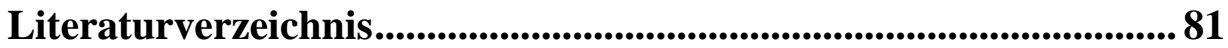

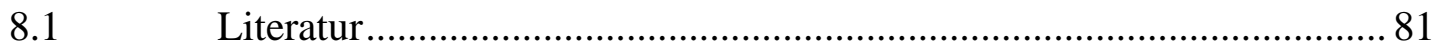

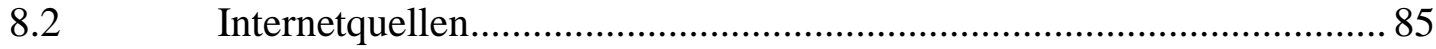




\section{Abbildungsverzeichnis}

Abbildung 1: Tibiofemoralgelenk von dorsal.

Abbildung 2: Gipsmodell in Originalgröße der artikulierenden Flächen eines rechten

Tibiofemoralgelenks ohne Menisken, in Extensionsstellung. ................................... 4

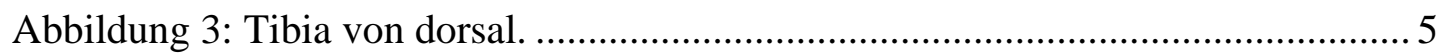

Abbildung 4: Femur, distaler Teil in der Ansicht von unten. .................................. 5

Abbildung 5: Muskeln der Hüfte und des Oberschenkels der rechten Seite in der Ansicht von vorne.

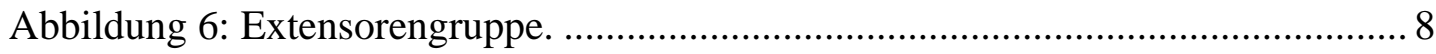

Abbildung 7: Flexorengruppe, Ansicht von dorsal............................................... 9

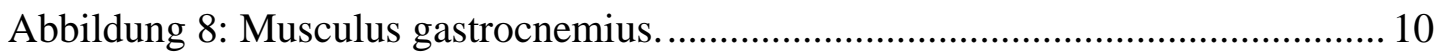

Abbildung 9: Musculus gastrocnemius, Ansicht dorsal.......................................... 11

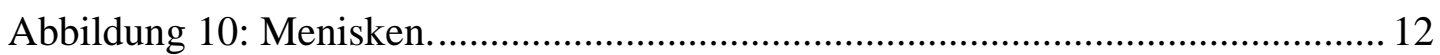

Abbildung 11: Menisken und Bänder. …............................................................. 14

Abbildung 12: Kniewinkel in der Flexion in Relation zur Stand- und Schwungphase des Gangzyklus. 16

Abbildung 13: Berechnetes Roll-Gleitverhalten des AEQOUS-Knies im medialen

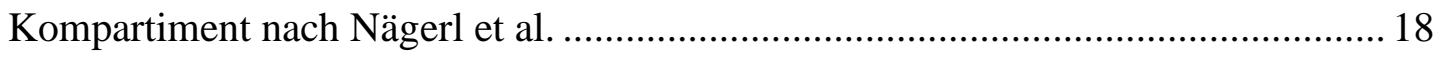

Abbildung 14: Mediales Kompartiment............................................................ 20

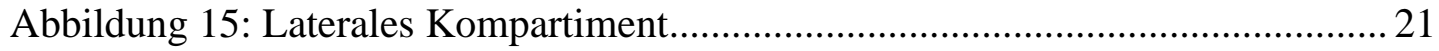

Abbildung 16: Dimere Ketten des Viergelenks.................................................. 22

Abbildung 17: Gleichgewichtszustände eines Viergelenks unter isotonischer Kraft.23 Abbildung 18: Frontale Ansicht des Tibiofemoralgelenks mit Kontaktpunkten....... 24

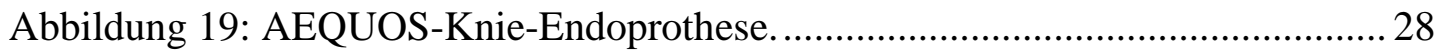

Abbildung 20: Konstruktion der Gelenkflächen des tibialen Inlays der AEQUOSEndoprothese.

Abbildung 21: Gangzyklus mit axialer Belastung des Kniegelenks......................... 30

Abbildung 22: Aufbau des Mebio Scan-Knies. 
Abbildung 23: Skizze der Messapparatur und des Kraftsystems nach Mackeben..... 35

Abbildung 24: Verbindungsteil der Kraftmessdose mit dem Kreisbogen. 36

Abbildung 25: Arretierung nach Knösel an der Kreisbogenschiene. 37

Abbildung 26: Messanordnung mit freischwebender Tibia im Gleichgewicht bei $5^{\circ}$ Kraftschluss, ohne Befestigung am Kreisbogen.

Abbildung 27: Messaufbau bei $90^{\circ}$ mit AEQUOS-Knie. .43

Abbildung 28: Messanordnung der Kraftmessdose.

Abbildung 29: AEQUOS-Knie, Femurkomponente (oben), Patellakomponente (Mitte), Tibiakomponente (unten). .46

Abbildung 30: Mebio Scan-Knie.. .47

Abbildung 31: Befestigung der Endoprothese.

Abbildung 32: Kraftschluss im Gelenk in Gleichgewichtslage mit eingezeichneten Ansätzen und Zug-richtungen der Kevlarseile.

Abbildung 33: Koordinatensystem in der Kraftmessdose und im Kniegelenk nach Gerstenkamp.

Abbildung 34: Detailaufnahme im Gleichgewichtszustand der Kreisbogenschiene mit Magneten zur Arretierung vor Auslenkung.

Abbildung 35: Resultierende Kraft und Drehmoment für die AEQUOS-Knie bei Auslenkungen im Flexionswinkel $5^{\circ}$, Messung 1.

Abbildung 36: Resultierende Kraft und Drehmoment für das AEQUOS-Knie bei Auslenkungen im Flexionswinkel $5^{\circ}$, Messung 2

Abbildung 37: Resultierende Kraft und Drehmoment für das AEQUOS-Knie bei Auslenkungen im Flexionswinkel $5^{\circ}$, Messung 5

Abbildung 38: Resultierende Kraft und Drehmoment für das AEQUOS-Knie bei Auslenkungen im Flexionswinkel $90^{\circ}$, Messung 6

Abbildung 39: Resultierende Kraft und Drehmoment für das AEQUOS-Knie bei Auslenkungen im Flexionswinkel $90^{\circ}$, Messung 7

Abbildung 40: Resultierende Kraft und Drehmoment für das Mebio Scan-Knie bei Auslenkungen im Flexionswinkel $5^{\circ}$, Messung 3. 
Abbildung 41: Resultierende Kraft und Drehmoment für das Mebio Scan-Knie bei Auslenkungen im Flexionswinkel $5^{\circ}$, Messung 4 65

Abbildung 42: Resultierende Kraft und Drehmoment für das Mebio Scan-Knie bei Auslenkungen im Flexionswinkel $90^{\circ}$, Messung 8

Abbildung 43: Resultierende Kraft und Drehmoment für das Mebio Scan-Knie bei Auslenkungen im Flexionswinkel $90^{\circ}$, Messung 9

Abbildung 44: Zusammenhang zwischen dem Kräfteverhältnis der Flexoren Hamstring zu Musculus gastrocnemius (Abszisse) und dem erzeugtem Drehmoment $\mathrm{T}^{\prime}(\mathrm{y})$ in Ncm/Grad (Ordinate). 69 162829314647 


\section{Tabellenverzeichnis}

Tabelle 1: Appliziertes Kräftesystem bei der Messung 1....................................... 58

Tabelle 2: Appliziertes Kräftesystem bei der Messung 2 ...................................... 59

Tabelle 3: Appliziertes Kräftesystem bei der Messung 5..................................... 60

Tabelle 4: Appliziertes Kräftesystem bei der Messung 6..................................... 62

Tabelle 5: Appliziertes Kräftesystem bei der Messung 7.......................................63

Tabelle 6: Appliziertes Kräftesystem bei der Messung 3........................................ 64

Tabelle 7: Appliziertes Kräftesystem bei der Messung 4..................................... 65

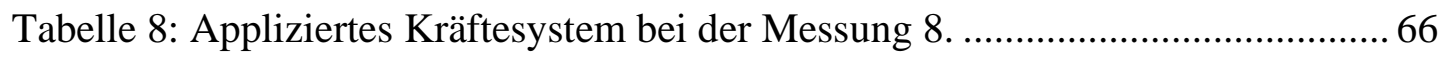

Tabelle 9: Appliziertes Kräftesystem bei der Messung 9..........................................6 68

Tabelle 10: Übersicht über die Muskelkräfte und Stabilitätsgrade...........................79

Tabelle 11: Übersicht über die Muskelkräfte und Stabilitätsgrade und Verhältnisse der

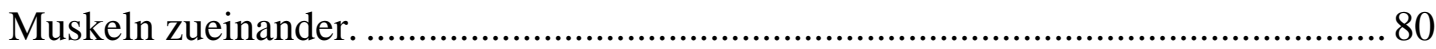




\section{Abkürzungsverzeichnis}

Art.

Articulatio

AWMF Arbeitsgemeinschaft der Wissenschaftlichen Medizinischen Fachgesellschaften

cm Zentimeter

CoCrMo Cobalt-Chrom-Molybdän-Legierung

DMS Dehnungsmessstreifen

$\mathrm{F}$

Femur

Lig. Ligamentum

M. Musculus

med. medial

$\mathrm{M}_{\mathrm{FL}} \quad$ lateraler Kurvaturenmittelpunkt des Femurs

MFM medialer Kurvaturenmittelpunkt des Femurs

mm Millimeter

$\mathrm{M}_{\mathrm{TM}} \quad$ medialer Kurvaturenmittelpunkt der Tibia

$\mathrm{M}_{\mathrm{TL}} \quad$ lateraler Kurvaturenmittelpunkt der Tibia

$\mathrm{N} \quad$ Newton

Ncm Newtonzentimeter

$\mathrm{R}_{\mathrm{FL}} \quad$ Radius der lateralen Kurvatur des Femurs

$\mathrm{R}_{\mathrm{FM}} \quad$ Radius der medialen Kurvatur des Femurs

$\mathrm{R}_{\mathrm{TL}} \quad$ Radius der lateralen Kurvatur der Tibia

$\mathrm{R}_{\mathrm{TM}} \quad$ Radius der medialen Kurvatur der Tibia

T Tibia

UHMWPE Ultrahochmolekulares Polyethylen 


\section{$1 \quad$ Einleitung}

In der Abhandlung der Gebrüder Weber aus dem Jahr 1836 wird bereits sehr anschaulich und ausführlich auf die morphologische Gestalt der femoralen und tibialen Kondylen des Kniegelenks und deren Bedeutung für die Funktion des Kniegelenks eingegangen. So stellten sie dar, dass das Kniegelenk in der Flexion/Extension wie ein Rad auf dem Boden rollen würde (Weber und Weber 1836). Die Gebrüder Weber beschrieben, dass die Krümmungen der femoralen Kondylen von vorne nach hinten zunähmen und dass eine Inkongruenz der Gelenkflächen bestünde, wobei Femur und Tibia sowohl im medialen als auch im lateralen Kompartiment sich in nur einem Punkte berührten. Somit zeigten sie schon 1836 wichtige morphologische Kriterien für die Funktionsweise des Tibiofemoralgelenks auf. Die in späteren Jahren vorgenommen Vereinfachungen der Kniegelenkmechanik im Sinne technischer Gelenke haben über lange Zeit wesentliche anatomische Voraussetzungen wie die Inkongruenz der Gelenkflächen und den Versatz der femoralen Achsen ignoriert und damit auch falsche Grundlagen für die Konzipierung künstlicher Knie-Endoprothesen geschaffen (Jerosch und Heisel 1999; Nägerl et al. 1993; Wetz und Jacob 2001).

Die Ansprüche an eine optimale Endoprothese betreffen neben der Schmerzreduktion vor allem Funktionalität im Hinblick auf die physiologischen Bewegungsabläufe. Da nicht nur die Zahl der Gonarthrosen steigt, sondern auch zunehmend alte und hochbetagte Menschen möglichst lange mobil bleiben möchten, ist der Bedarf an Kniegelenkprothesen hoch (Kirschner 2011). In diesem Zusammenhang gewinnen anatomisch geformte Implantate und auch der Gelenkflächenteilersatz an Bedeutung. Bisher gibt es nur wenige Implantate auf dem Markt, die in der Lage sind, den natürlichen Roll/Gleitmechanismus des Kniegelenks wiederzugeben.

Im natürlichen Kniegelenk kann in der Sagittalebene eine gegebene momentane Lage des Gelenks durch Veränderung der Richtung der Kraft des angreifenden muskulären Systems der Gleichgewichtszustand von Stabilität über Indifferenz bis zur Instabilität eingestellt werden (Nägerl et al. 1993). So kann der Mensch in einer Hockposition ein stabiles Gleichgewicht halten, aber auch durch eine Änderung des Kraftvektors der Muskulatur im Kniegelenk ein instabiles Gleichgewicht erzeugen, welches das Aufstehen aus der Hocke ermöglicht.

Die vorliegende Arbeit hat zum Ziel, die Ergebnisse der Messungen zur mechanischen Stabilität zweier Endoprothesensysteme zu vergleichen und zu bewerten. Im Vergleich 
zum AEQUOS-Knie wird das Mebio Scan-Knie untersucht, welches sich durch lateral/mediale symmetrische Oberflächengestaltung der beiden Gelenkkompartimente auszeichnet.

Vorangehend erfolgt eine Übersicht der anatomischen Strukturen des Tibiofemoralgelenks und des Gangzyklus des Menschen. Nach der Vorstellung der Endoprothesen wird die spezielle Mechanik des Kniegelenks in Flexion/Extension unter Berücksichtigung des Viergelenks und der Bedeutung der geometrischen Gestalt der artikulierenden Flächen für die Kinematik des Tibiofemoralgelenks erläutert.

Kapitel 3 stellt die Methodik und die Messapparatur sowie den Versuchsablauf dar, bevor in Kapitel 4 die Ergebnisse beschrieben und anschließend diskutiert werden. 


\section{$2 \quad$ Anatomie und Biomechanik des Kniegelenks}

\subsection{Anatomie des Tibiofemoralgelenks}

\subsubsection{Gelenkflächen}

Das Kniegelenk umfasst die Articulatio femoropatellaris und die Articulatio femorotibialis. Die erstere, die zwischen den Knochen Femur und Patella artikuliert, stellt eine Art „Umlenkrolle“ dar, mit der die Kraft des Musculus quadriceps femoris auf die Tibia umgelenkt wird. Die Articulatio femorotibialis ist zusammengesetzt aus einem lateralen und einem medialen Gelenkkompartiment. Femur und Tibia berühren sich in zwei punktförmigen Kontakten.

Die für die Kniefunktion entscheidenden Strukturen stellen die artikulierenden Flächen auf den medialen und lateralen Condyli femoris und tibiae des Tibiofemoralgelenks (Abbildung 1) dar.

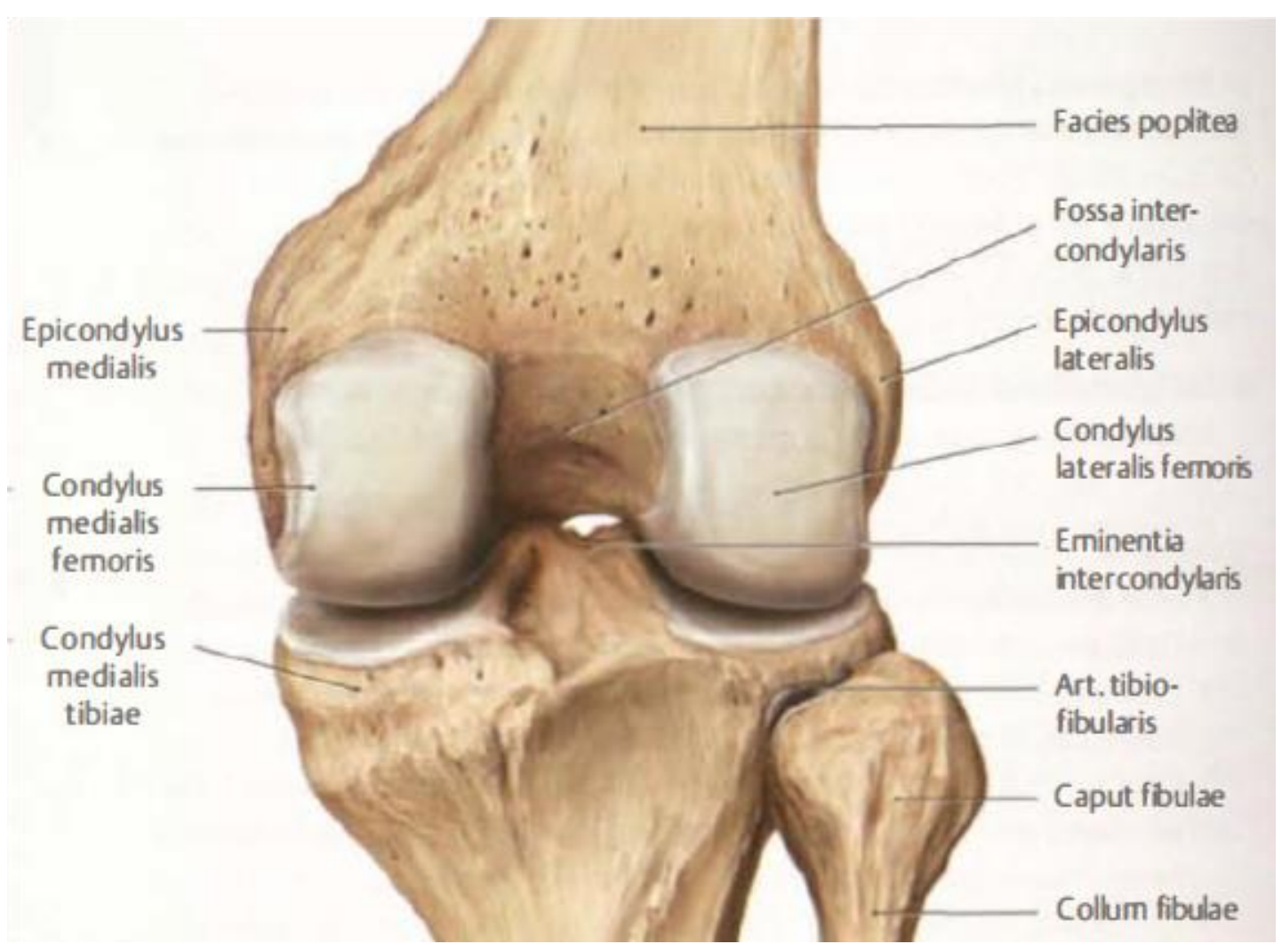

Abbildung 1: Tibiofemoralgelenk von dorsal (Prometheus 2014). Der Abdruck erfolgt mit freundlicher Genehmigung des Georg Thieme Verlages.

Die Tibia besteht aus dem Corpus tibiae und dem Caput tibiae, welches das proximale 
Endstück bildet. Die für die Artikulation der Tibia mit dem Femur wesentlichen Strukturen sind kranial der Condylus medialis und der Condylus lateralis des Caput tibiae.

Die Kondylen der Tibia sind wie die des Femurs von knorpeligen Gelenkflächen überzogen, den Facies articularis superiores.

Der Knorpel besteht aus extrazellulärer Matrix, die zu 15-22 \% aus Kollagenfasern, das heißt aus Mikrofibrillen des Kollagens Typ II, und zu 4-7 \% aus Proteoglycanen in erster Linie Aggrecan - besteht (Mow et al. 2005). Im Knorpel ist die Synovia eingelagert. Der Wasseranteil beträgt 60-85 \%. Unter statischer Belastung tritt Wasser aus der Matrix aus.

Zwischen den Kondylen verläuft sagittal die Eminentia intercondylaris, die von zwei abgeflachten Hügeln gebildet wird, den Tubercula intercondylare mediale und laterale. In ihnen entspringen die Kreuzbänder.

Das laterale Tibiaplateau ist in der Sagittalen konvex gekrümmt und in der Transversalen konkav, das mediale Plateau ist aber sowohl in der Transversalen als auch in der Sagittalen konkav geformt, mit einer größeren Gesamtfläche als auf der lateralen Seite. Die unterschiedliche Wölbung der Kondylen ist ein entscheidendes Faktum für die spezielle Mechanik des Kniegelenks und wird in Kapitel 2.3.2 näher betrachtet. Hierbei ist von besonderer Bedeutung, dass die Kontaktpunkte auf den Abhängen der Eminentia liegen (Fiedler et al. 2011).

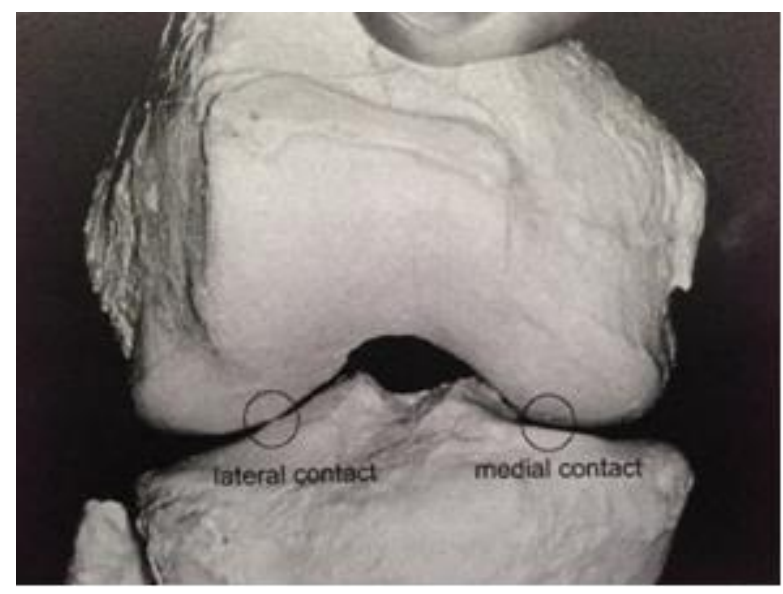

Abbildung 2: Gipsmodell in Originalgröße der artikulierenden Flächen eines rechten Tibiofemoralgelenks ohne Menisken, in Extensionsstellung (Fiedler et al. 2011). Der Abdruck erfolgt mit freundlicher Genehmigung von Dr. C. Fiedler.

Zu erkennen ist die Positionierung des lateralen Kontaktpunktes auf dem Abhang des Tuberculum condylare. Der mediale Kontaktpunkt ist in dieser Ansicht nicht direkt abgebildet. 


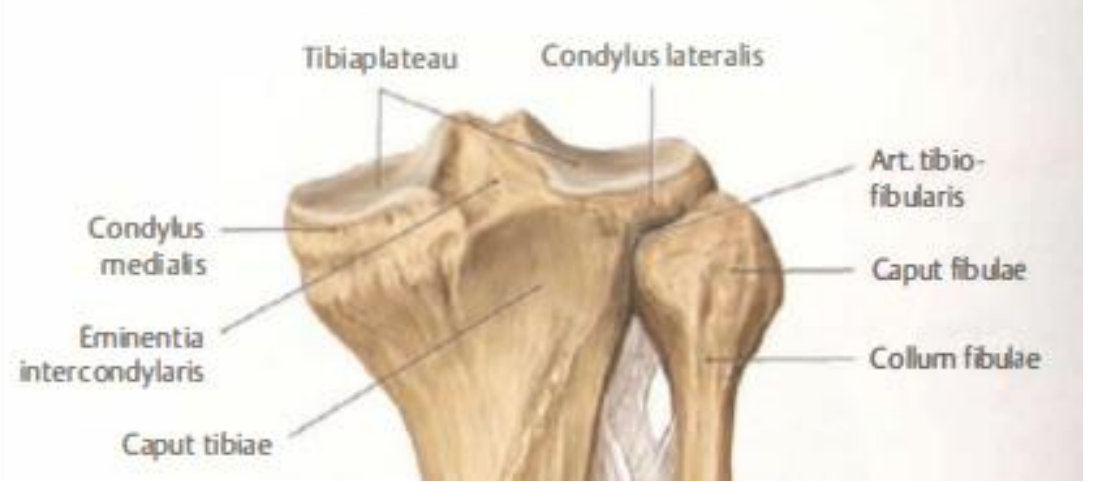

Abbildung 3: Tibia von dorsal (Prometheus 2014). Der Abdruck erfolgt mit freundlicher Genehmigung des Georg Thieme Verlages.

Das Femur ist der größte Röhrenknochen des menschlichen Skeletts. Sein distales Ende weist drei Gelenkflächen auf, von denen zwei mit der Tibia artikulieren: der mit Knorpel überzogene Condylus medialis und der Condylus lateralis. Beide Gelenkflächen sind konvex gekrümmt und laufen nach vorn in einer sattelähnlichen Fläche zusammen. Bei genauer Betrachtung der Kondylen fällt ihre stärkere sagittale konvexe Krümmung im lateralen Kompartiment verglichen mit der im medialen Kompartiment auf (Vergleiche Abbildung 4, Abbildung 14 und Abbildung 15).

Zwischen den Kondylen verläuft die Fossa intercondylaris, sie bietet den Kreuzbändern Platz. Die Epicondyli medialis und lateralis stellen den Ansatzpunkt der Seitenbänder des Kniegelenks dar (Fanghänel et al. 2003).

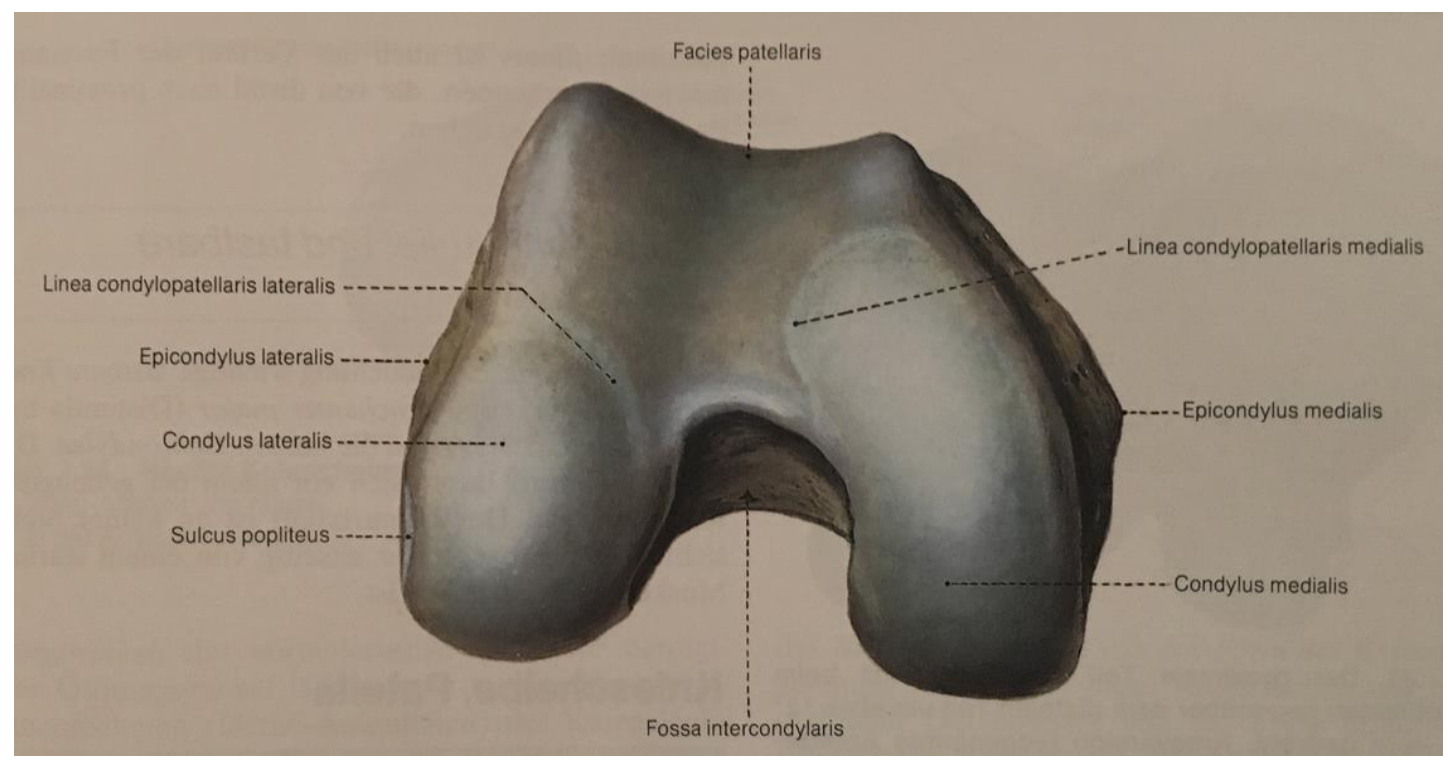

Abbildung 4: Femur, distaler Teil in der Ansicht von unten (Tillmann 2003). Der Abdruck erfolgt mit freundlicher Genehmigung des Georg Thieme Verlages. 
Des Weiteren befindet sich weiter ventral eine sattelförmige, überknorpelte Vertiefung, die Facies patellaris, die dritte Gelenkfläche, die mit der Patella artikuliert.

\subsubsection{Muskel- und Bandapparat}

\subsubsection{Muskulatur}

Die Muskulatur des Kniegelenks hat sowohl eine statische als auch eine dynamische Aufgabe. Zum einem kann sie bei vorgegebener Gelenkposition die Qualität der Gelenkstabilität durch Rotation des Gelenkkraftvektors um die momentane Drehachse des Gelenks verändern. Zum anderen erzeugen die Muskeln zusammen mit der Schwerkraft den Kraftschluss im Gelenk, welcher eine zwingende Voraussetzung für die suffiziente kinematische/biomechanische Funktion des Tibiofemoralgelenks ist (Nägerl et al. 1993).

Allgemein gesprochen sollen die Muskeln des Kniegelenks die Relativbewegungen von Femur, Patella und Tibia zueinander sowie die Stabilität bei statischen Positionen mit unterschiedlichem Kniewinkel, unter anderem beim Stehen und Hocken, ermöglichen.

Die Extension wird aktiv durch die Extensorengruppe der beteiligten Muskulatur, bestehend aus den vier Köpfen des Musculus quadriceps femoris, herbeigeführt.

Einen der Köpfe bildet der Musculus rectus femoris, welcher vom Vorderrand des Darmbeins und vom oberen Rand der Hüftgelenkpfanne entspringt. Daneben gibt es noch die drei Musculi vasti, welche nicht vom Becken entspringen, sondern vom Femur ausgehen, dabei umhüllen sie diesen von vorne und von den Seiten. Somit zieht der Musculus rectus femoris sowohl über das Hüft- als auch über das Kniegelenk, und die restlichen drei Köpfe ziehen lediglich über das Kniegelenk (Abbildung 5). 


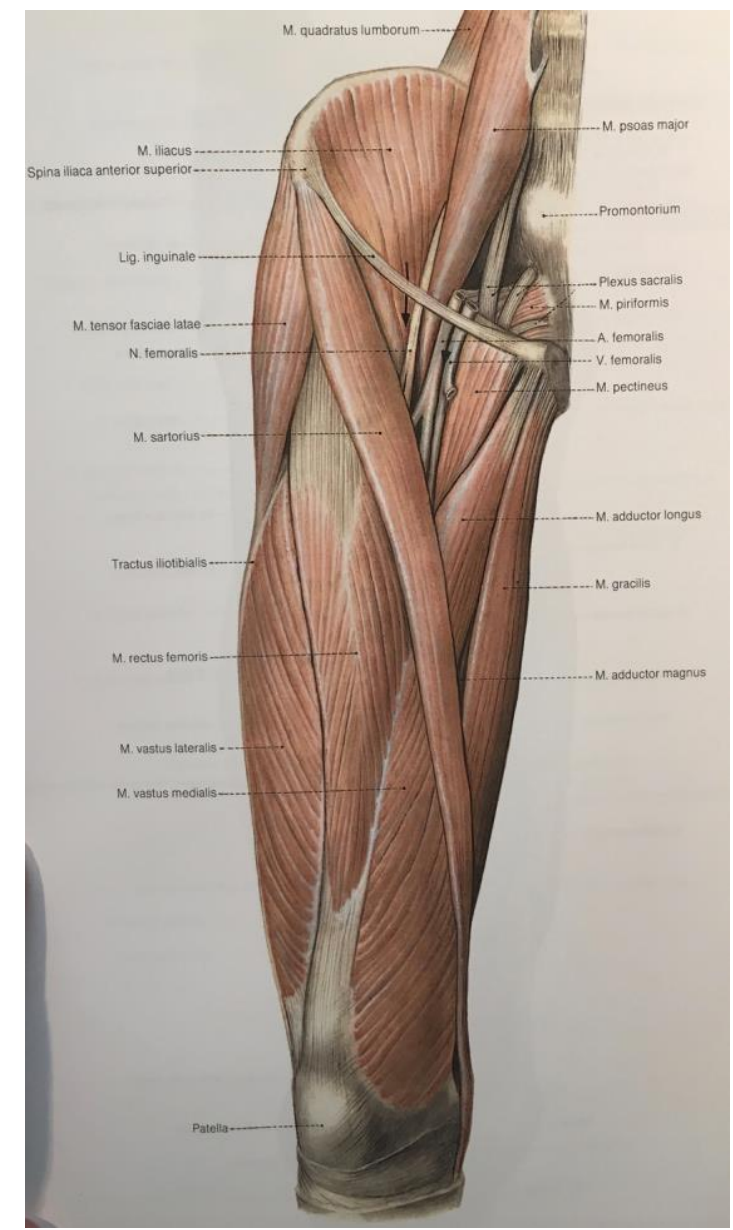

Abbildung 5: Muskeln der Hüfte und des Oberschenkels der rechten Seite in der Ansicht von vorne (Tillmann 2003). Der Abdruck erfolgt mit freundlicher Genehmigung des Georg Thieme Verlages.

Die genaue Topographie der drei weiteren Köpfe ist wie folgt: Die Musculi vasti medialis und lateralis entspringen von der Linea intertrochanterica und den Labia mediale und laterale der Linea aspera femoris (Linea intertrochanterica $=$ raue Linie vorn zwischen Schaft und Hals des Femurs, zieht vom Trochanter major zum Trochanter minor; Linea aspera = raue Doppellinie an der Femurrückseite, circa in der Mitte).

Besonders zu erwähnen ist der Musculus vastus intermedius, welcher von der Vorderfläche des Femurs unterhalb der beiden oberen entspringt, da ein Teil seiner Fasern, die weit distal entspringen, einen eigenen Faserzug bilden, den Musculus articularis genus. Dessen Funktion besteht darin, die Gelenkkapsel des Knies zu spannen und so ihre Einklemmung bei der Streckung zu verhindern (Schünke 2000).

Alle vier Köpfe enden in einer gemeinsamen Endsehne, dem Ligamentum patellare, in dem die Patella eingelagert ist und das ventral über das Kniegelenk zieht (Kapandji 2009). 


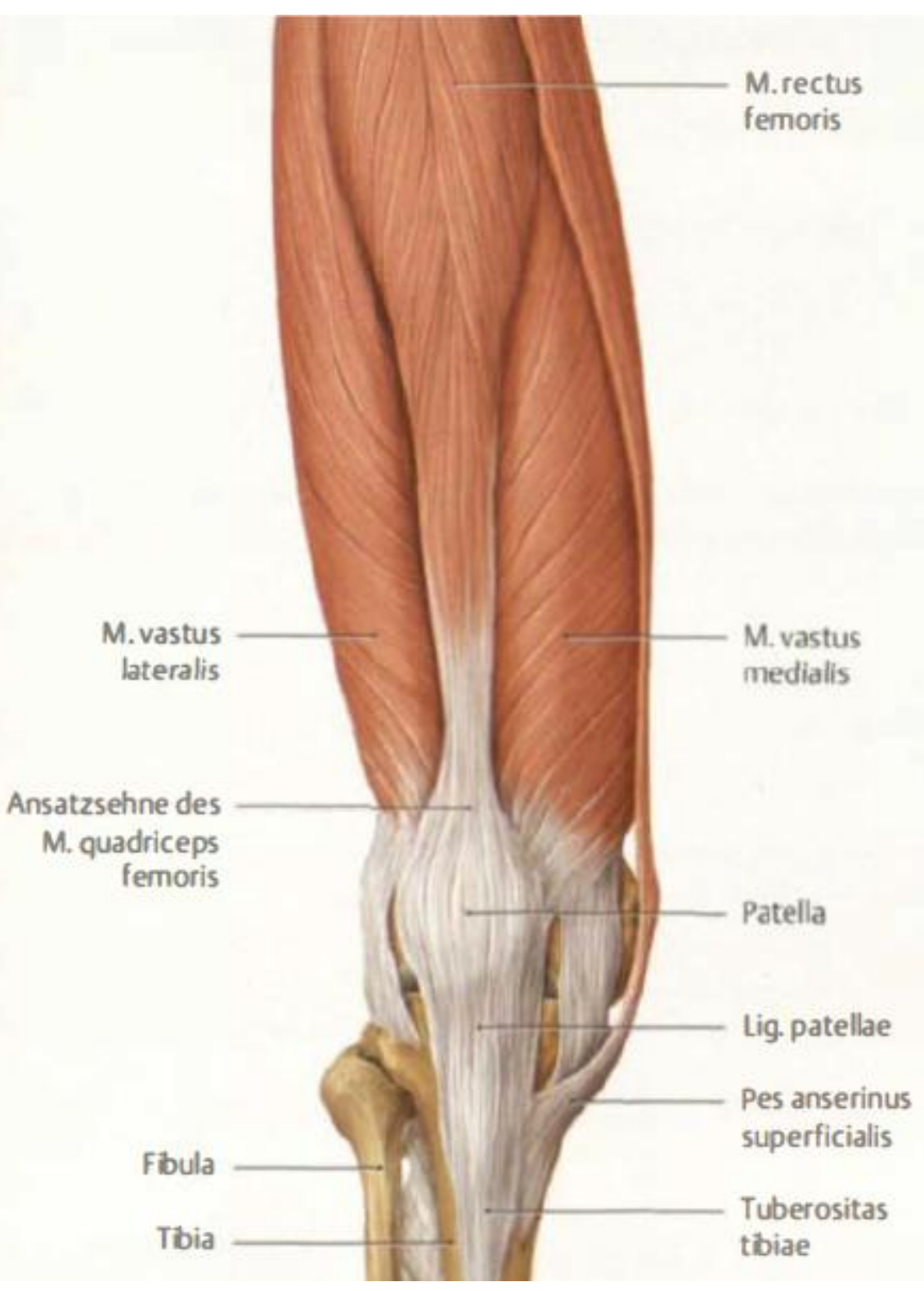

Abbildung 6: Extensorengruppe (Prometheus 2014). Der Abdruck erfolgt mit freundlicher Genehmigung des Georg Thieme Verlages.

Die Muskeln, die an der Flexion beteiligt sind, lassen sich aufteilen in die Hauptmuskelgruppe der ischiokruralen Muskeln oder Hamstrings, die Muskeln des Pes anserinus und die Muskeln der Wadenmuskulatur, die an der Flexion des Kniegelenks teilnehmen.

Die ischiokrurale Muskelgruppe besteht aus dem Musculus biceps femoris, dem Musculus semimembranosus und dem Musculus semitendinosus. Sie alle entspringen vom Tuber ischiadicum an der Rückseite des Oberschenkels. Das Caput longum des Musculus biceps femoris strahlt von hier nach lateral aus und vereinigt sich mit seinem Caput breve, welches von der Linea aspera kommt. Gemeinsam enden sie am Caput 
fibulae.

Während der Musculus biceps femoris für Flexion und Außenrotation der Tibia verantwortlich ist, steuern die Musculi semimembranosus und semitendinosus die Innenrotation (Schünke 2000).

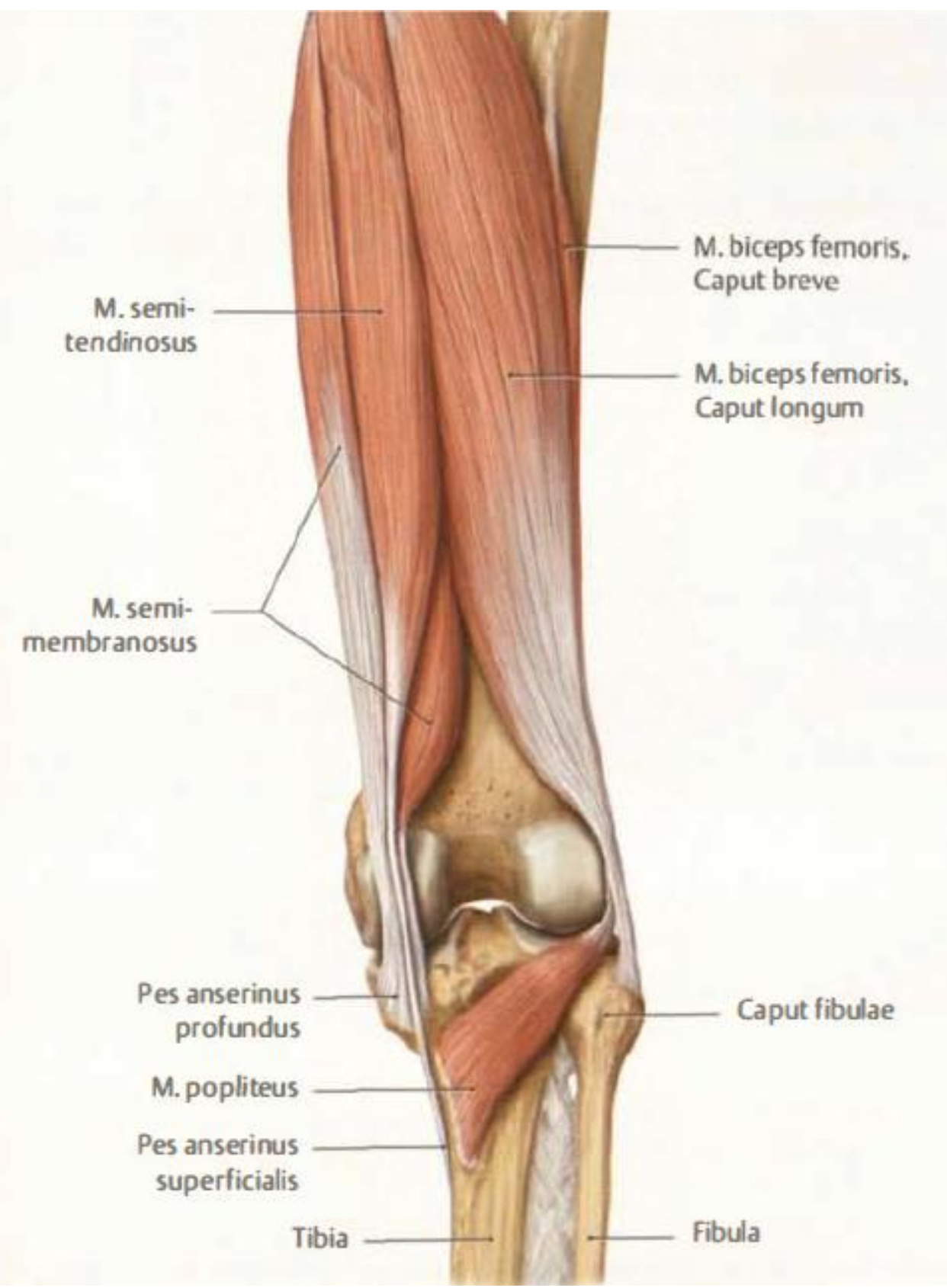

Abbildung 7: Flexorengruppe, Ansicht von dorsal (Prometheus 2014). Der Abdruck erfolgt mit freundlicher Genehmigung des Georg Thieme Verlages.

Der Musculus sartorius nimmt eine Sonderstellung ein: Er zählt zwar zu der vorderen Muskelgruppe des Oberschenkels, da er vom vorderen Ende des Darmbeinkamms aus 
über den Musculus quadriceps femoris hinwegläuft, doch bestimmt sein Ansatz am Pes anserinus seine Funktion als Beuger (Fanghänel et al. 2003). Ebenso beugt der Musculus gracilis, welcher vom unteren Rand des Schambeins und der ventralen Seite der Symphyse aus seitlich bis zur Tibia verläuft.

An der Beugung des Kniegelenks nehmen des Weiteren die Muskeln Popliteus und Gastrocnemius der Wadenmuskulatur teil.

Der Musculus popliteus entspringt am Condylus lateralis femoris und läuft in der Kniekehle zur Facies posterior tibiae (Abbildung 7). Die meisten modernen Lehrbücher zählen den Musculus popliteus zu den Extensoren, doch wie bereits die Brüder Weber 1836 erkannten, kontrolliert er ebenfalls die Flexion des Knies, neben seiner besonderen Funktion für die Innenrotation des Kniegelenks (Nägerl et al. 1993; Bizzini 2000). Durch seinen Ursprung sowohl am Condylus lateralis femoris als auch am Hinterhorn des lateralen Meniskus, wie auch über das Ligamentum popliteum arcuatum vom Caput fibulae, kann der Musculus popliteus seine Aufgaben bei der Innenrotation und Flexion erfüllen (Fanghänel et al. 2003).

Der Musculus gastrocnemius inseriert mit dem Caput laterale am Condylus lateralis und mit dem Caput mediale am Condylus medialis femoris (Fanghänel et al. 2003) (Abbildung 8). Er reguliert auch die Plantarflexion (Abbildung 9).

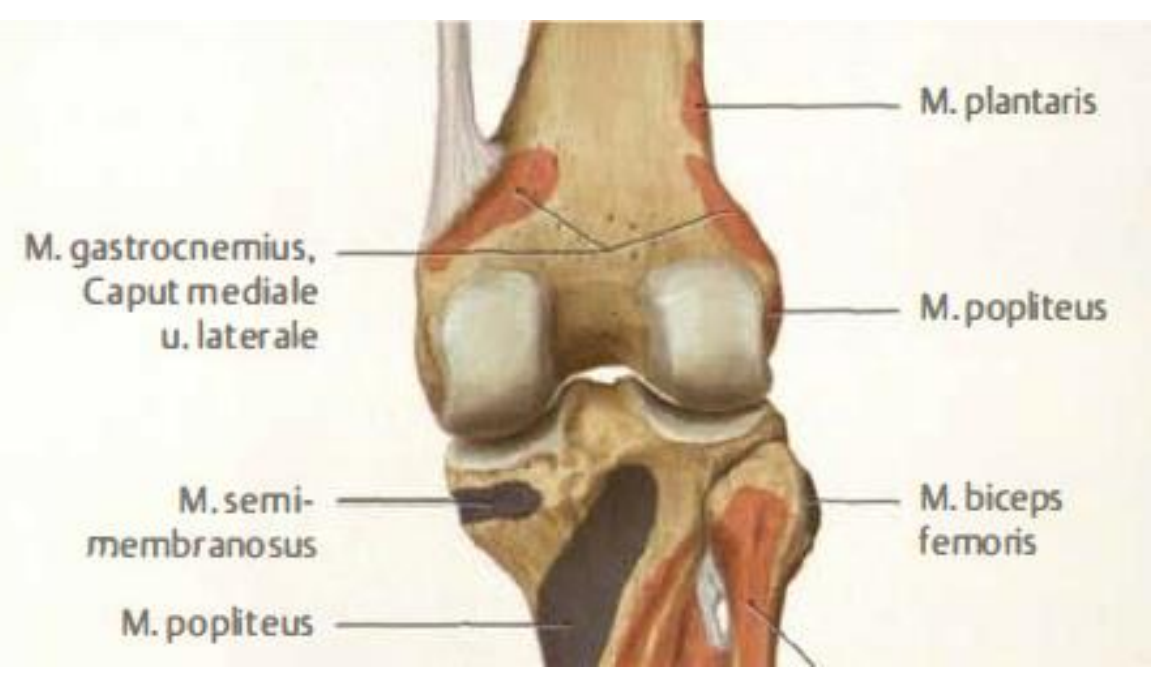

Abbildung 8: Musculus gastrocnemius (Prometheus 2014). Der Abdruck erfolgt mit freundlicher Genehmigung des Georg Thieme Verlages. 


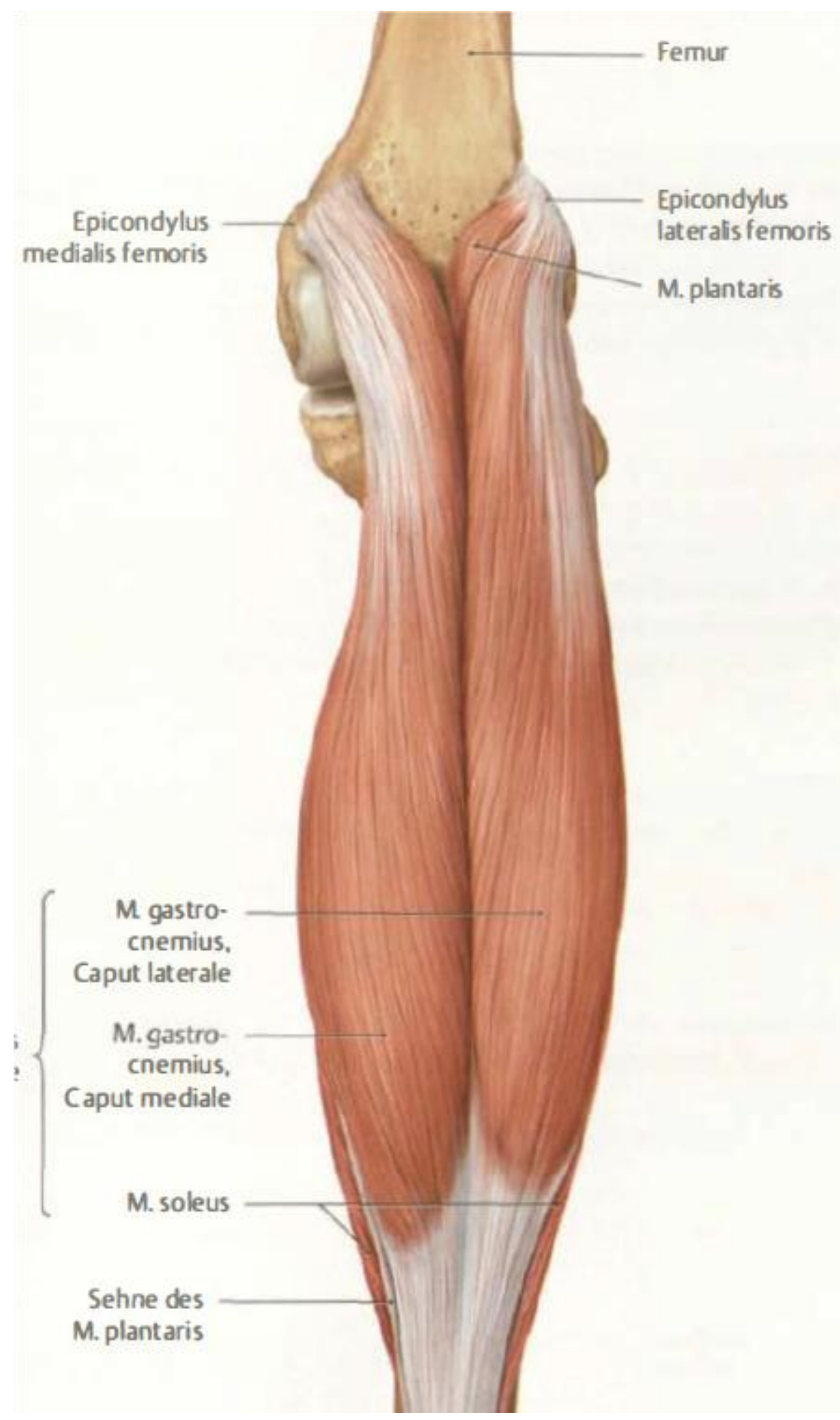

Abbildung 9: Musculus gastrocnemius, Ansicht dorsal (Prometheus 2014). Der Abdruck erfolgt mit freundlicher Genehmigung des Georg Thieme Verlages.

An der Außenrotation des Kniegelenks ist vor allem der Musculus biceps femoris zusammen mit dem Gastrocnemius beteiligt, wohingegen die Innenrotation durch die Musculi semimembranosus, semitendinosus, popliteus, sartorius und graciles provoziert wird. 


\subsubsection{Menisken}

Zwischen den artikulierenden Flächen von Tibia und Femur liegen zwei Keile aus Knorpelmasse mit konvexer Wölbung zum Femur und flacher Auflage zur tibialen Seite: die Menisken (Meniscus medialis, Meniscus lateralis). Der mediale Meniskus bildet die Form eines offenen C (Abbildung 10), der laterale Meniskus stellt einen fast geschlossenen Halbkreis dar. Beide Menisken sind sowohl im anterioren als auch im posterioren Bereich mit der Area intercondylaris verbunden. Der mediale Meniskus bildet darüber hinaus eine feste Verbindung mit dem Lig. collaterale tibiale, wogegen der laterale Meniskus auf der Membrana synovialis der Gelenkkapsel verankert ist (Abbildung 10) (Athanasiou und Sanchez-Adams 2009).

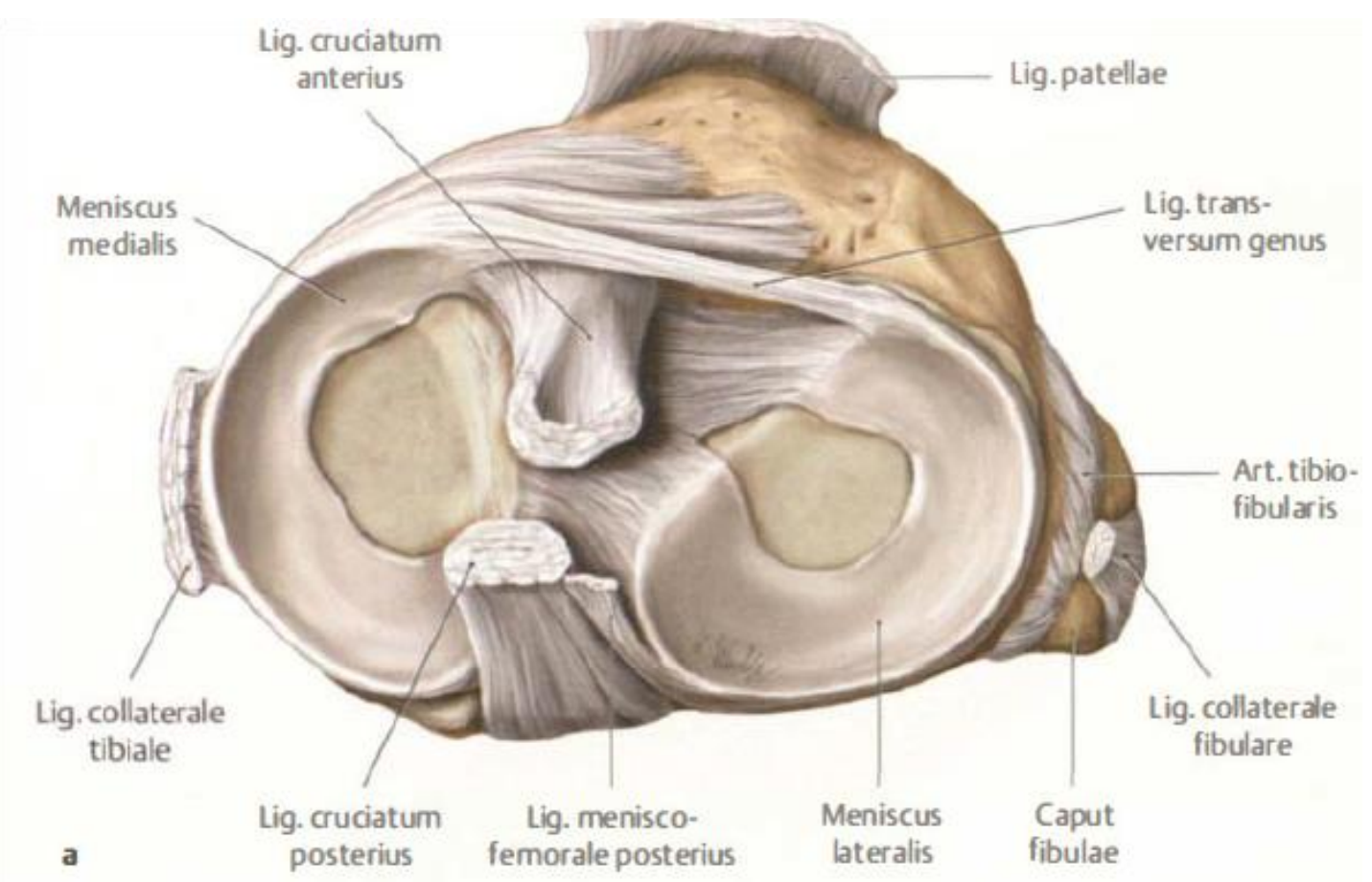

Abbildung 10: Menisken (Prometheus 2014). Der Abdruck erfolgt mit freundlicher Genehmigung des Georg Thieme Verlages.

Eine Hauptaufgabe der Menisken besteht in der Verkleinerung und Abdichtung des Gelenkraumes gegenüber Über- und Unterdruck. Durch diese Gelenkspaltveränderung zusammen mit dem durch Last evozierten Wasseraustritt aus dem Knorpel wird die Schmierung der artikulierenden Flächen realisiert (Fanghänel et al. 2003).

Die Menisken haben keine direkte kinematische Funktion (Nägerl et al. 1993; Athanasiou und Sanchez-Adams 2009), sondern vielmehr haben Studien ergeben, dass die 
Propriozeption unter anderem über die Innervation der Menisken mit Nozizeptoren und Mechanorezeptoren im äußeren Bereich gesteuert wird. Ruffini-, Vater-Paciniund Golgi-Rezeptoren wurden insbesondere in den tibialen Aufhängungen der Menisci nachgewiesen (Blümel und Kraatz 1997; Nägerl et al. 2002), die Signale an das zentrale Nervensystem zur momentanen Position des Gelenks senden (Day et al. 1985; Zimny et al. 1988; Gray 1999; Saygi et al. 2005) und darüber auch eine Kooperation der Menisken mit den Kreuzbändern möglich machen (Solomonow et al. 1987). Die Mechanorezeptoren befinden sich zwischen den Fasern der ligamentären Aufhängungen der Menisken und werden aktiv, wenn sich benachbarte Fasern unterschiedlich dehnen. Dieser Übergang vom Bereich gedehnter Fasern zum Bereich der nichtgedehnten Fasern hängt von der Relativposition von Tibia und Femur ab (Nägerl et al. 2002).

\subsubsection{Bänder}

Die Bänder werden unterschieden in die sogenannten Innenbänder und die Außenoder Kollateralbänder. Dabei verlaufen die Außenbänder außerhalb der Gelenkkapsel, die Innenbänder innerhalb der Gelenkkapsel, aber außerhalb der Gelenkhöhle (Abbildung 11).

Das Ligamentum collaterale tibiale entspringt am Epicondylus medialis femoris und inseriert mit seinen langen vorderen Faserzügen am Schienbein und mit den kürzeren hinteren am Meniscus medialis. Das Ligamentum collaterale fibulare zieht vom Epicondylus lateralis femoris zum Caput fibulae. Es wird durch die Sehne des Musculus popliteus von der Gelenkkapsel getrennt. Bei Streckung und Außenrotation sind die Kollateralbänder gespannt und bei Beugung und Innenrotation entspannt. Sie begrenzen somit Ab- und Adduktion (Schünke 2000).

Die Innenbänder stellen die Kreuzbänder dar. Die Kreuzbänder spannen sich bei Extension an. Das vordere Kreuzband, Lig. cruciatum anterius, strahlt von der Innenfläche des lateralen Kondylus des Femurs in die Area intercondylaris anterior auf der Tibia. Auffallend ist, dass das vordere Kreuzband in sich selbst verwunden ist. Es ist bei der Innenrotation gespannt.

Das hintere Kreuzband, Lig. cruciatum posterius, erstreckt sich von der Area intercondylaris posterior der Tibia bis zur Innenfläche des medialen Condylus des Femurs. Es lässt sich in zwei Faserbündel aufteilen, ein medial vorderes und ein medial hinteres. 
Das hintere Faserbündel ist bei Flexion gespannt und in Extension entspannt.

Die in den Kreuzbändern vorhandenen Ruffini- und Vater-Pacini-Körperchen stellen das Sensorsystem dar, mittels dem die Kreuzbänder vor allem die FlexionsExtensionsbewegung des Tibiofemoralgelenks registrieren können, welche aus einer Rotation und einer Translation zusammengesetzt ist (Nägerl et al. 2002).

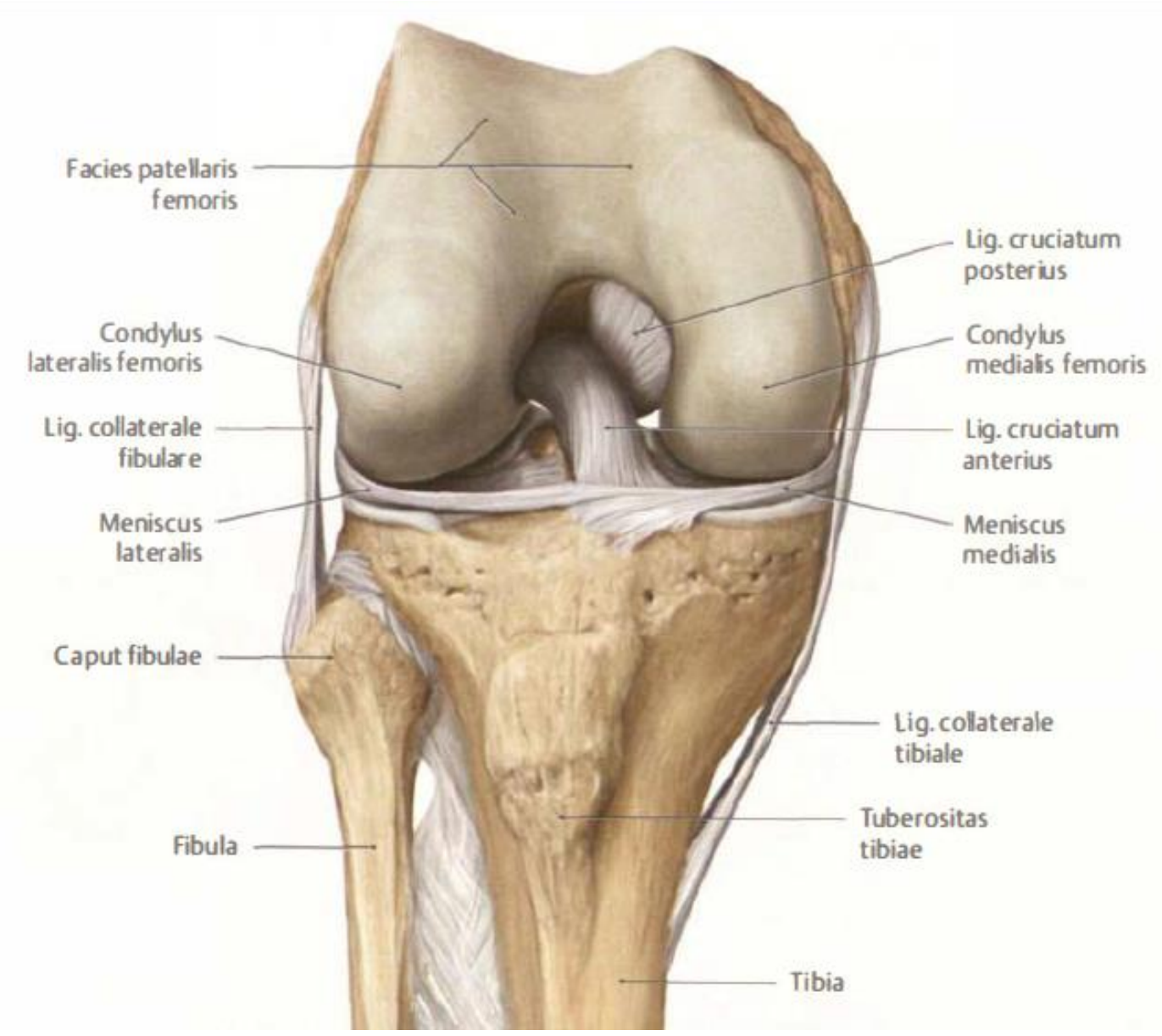

Abbildung 11: Menisken und Bänder (Prometheus 2014). Der Abdruck erfolgt mit freundlicher Genehmigung des Georg Thieme Verlages.

In den Kreuzbändern finden sich Mechanorezeptoren, deren Propriozeption entscheidend ist für die Biomechanik des Kniegelenks (Tillmann 2003; Halata und Haus 1989; Nägerl et al. 1993; Dyhre-Poulsen und Krogsgaard 2000).

Menschik (1974) beschrieb die Funktion des Kniegelenks als Viergelenk gegeben durch die Kreuzbänder; bereits 1917 jedoch beschrieb Strasser diese Annahme als nichtzutreffend (Menschik 1974; Strasser 1917). Nägerl widerlegte 2002 Menschik nochmals ausdrücklich und sprach den Kreuzbändern die Hauptaufgabe zu, durch 
sensorische Leistung den zwingend für die korrekte kinematische Funktion des Kniegelenks erforderlichen Kraftschluss zwischen Femur und Tibia zu gewährleisten.

Seine Aussage (Nägerl 2002) stützt sich auch auf die Ergebnisse anderer Autoren (Schutte et al. 1987; Zimny et al. 1988; Schultz et al. 1984; Johansson 1991; Katonis et al. 1991), die darlegen, dass es zahlreiche Mechanorezeptoren in den Bändern gibt. Der polysynaptische Reflexbogen, der dies verwirklicht, besteht aus den Mechanorezeptoren und sensiblen Nervenendigungen in den Kreuzbändern, welche den afferenten Schenkel des Reflexes repräsentieren und zum Rückenmark führen. Hier findet eine Verschaltung auf den efferenten Schenkel statt. Dieser wird gebildet aus $\alpha$-Motoneuronen und $\Gamma$-Motoneuronen, welche die Arbeitsmuskulatur beeinflussen oder in den Muskelspindeln enden. Ebenso ist eine Verschaltung auf Kollaterale möglich, die eine hemmende Wirkung auf die Arbeitsmuskulatur haben. Durch diese Verknüpfung ist eine Kraftsteuerung realisiert.

Die Kreuzbänder können nach Nägerl et al. (2002) nicht nur den Kraftschluss registrieren, sondern auch den Flexionsgrad des Gelenks, um die Muskulatur optimal steuern zu können.

\subsection{Der Gangzyklus}

\subsubsection{Ablauf und Schema}

Der Gangzyklus ist durch die kooperierende Bewegung des Standbeins, auch Stemmbein genannt, und des Schwungbeins oder Spielbeins gekennzeichnet. Die gesamte Dauer eines Gangzyklus umfasst die Zeit vom Beginn des ersten Fersenkontaktes eines Beines bis zum nächsten Fersenkontakt.

So kann man den Gangzyklus aufteilen in eine Stand- oder Stützphase und eine Schwungphase. In der internationalen Literatur wird in den letzten zehn Jahren überwiegend die Nomenklatur des Rancho Los Amigos National Rehabilitation Center (RLANRC), Los Angeles, verwendet, die auch in der vorliegenden Untersuchung genutzt wird.

Die Standphase beginnt mit der initialen bipedalen Phase, geht über in die monopedale Phase mit dem Berühren der Ferse des rechten Beines am Boden und wird beendet durch das Abstoßen der Zehen. In dieser Phase setzt das andere Bein bereits mit der 
Ferse auf. Hier beginnt die Schwungphase, welche mit dem Abheben des rechten Fußes beginnt und bis zum erneuten Bodenkontakt der rechten Ferse andauert (Mitchell und Galen Mitchell 2005; Götz-Neumann 2006). Dabei übernimmt das jeweilige Standbein das Körpergewicht, sobald sich das Schwungbein ohne Kontakt zum Boden befindet. Diese Phase wird auch als einfach unterstützte Standphase bezeichnet (GötzNeumann 2006).

Die erweiterte Standphase, zu der auch die Phase einer doppelseitigen Abstützung gehört, nimmt etwa 60 \% der gesamten Zykluszeit ein. Die Phase des Gehens, in der eine doppelseitige Abstützung stattfindet, erstreckt sich vom Abheben der Zehen des Schwungbeins bis zum Verlust des Bodenkontaktes der Zehen des gegenläufigen anderen Beines, dem Standbein (Perry 2003; Götz-Neumann 2006). In Abhängigkeit von der Geschwindigkeit des Gehens variiert die Phase der doppelseitigen Beinbelastung. Diese wird minimiert oder entfällt gänzlich, je schneller der Gang ist, so zum Beispiel beim Sprinten.

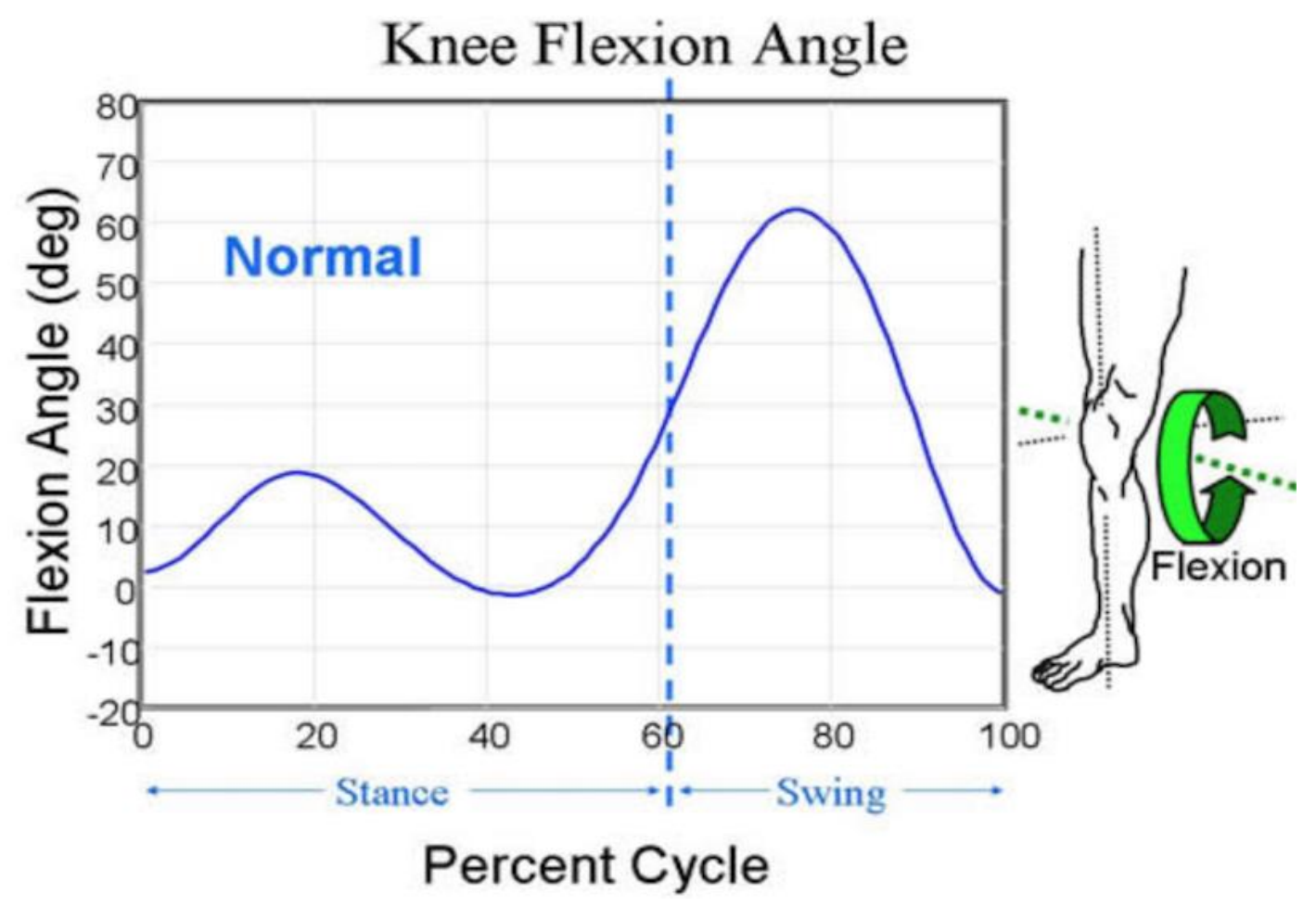

Abbildung 12: Kniewinkel in der Flexion in Relation zur Stand- und Schwungphase des Gangzyklus, (http://my.me.queensu.ca/People/Deluzio/DataAnalysis.html; Zugriff am 11.04.2018).

In Abbildung 12 werden die Flexionswinkel des Kniegelenks im Gangzyklus dargestellt. 


\subsubsection{Aufgabe des Kniegelenks im Gangzyklus}

In dem prozentual großen Anteil der Standphase am Gangzyklus ist das Kniegelenk besonders beteiligt, da es hier sowohl eine kinematische Funktion erfüllt als auch gleichzeitig Kraftübertragung leisten muss. Die Standphase beginnt mit der Extension des Kniegelenks und der Gewichtsübernahme. Beginnende Knieflexion kennzeichnet die Belastungsphase. Die Standphase endet wieder mit gestrecktem Kniegelenk und leitet über in die Schwungphase.

Das Kniegelenk ist in der Phase der Gewichtsübernahme deutlich mehr möglichem Verschleiß ausgesetzt als in der Schwungphase, in der zwar das Kniegelenk bewegt wird, dies jedoch ohne Belastung durch das Körpergewicht geschieht. Auch in der Schwungphase werden Muskelkräfte aktiv, so stellen Musculus vastus medialis und Musculus biceps femoris den Kraftschluss und damit die Gelenkführung durch die artikulierenden Flächen her (Mitchell und Galen Mitchell 2005).

Daraus lässt sich folgern, dass es für den Verschleiß des Kniegelenks, vor allem der Knorpelschicht beziehungsweise des Materials einer Endoprothese, entscheidend ist, in dieser Phase einen physikalisch begründeten geringen Abrieb zu haben. Dies wird durch das überwiegende Rollen der Artikulationsflächen in der Standphase verwirklicht. Durch das Rollen wird die Gleitreibung durch die geringere Rollreibung ersetzt (Nägerl et al. 2008).

Den einzelnen Phasen des Ganges können Flexionswinkel des Kniegelenks zugeordnet werden, wie sie in Abbildung 12 dargestellt sind. So beginnt die sogenannte pre-swingPhase oder initiale Stützphase bei Winkeln von $5^{\circ}$ bis $15^{\circ}$. In der initialen Schwungphase durchläuft das Kniegelenk Flexionswinkel von $40^{\circ}$ bis $60^{\circ}$, die sich bis zur mittleren Schwungphase auf $25^{\circ}$ verringern und in der terminalen Schwungphase bei $5^{\circ}$ auskommen (Götz-Neumann 2006). 


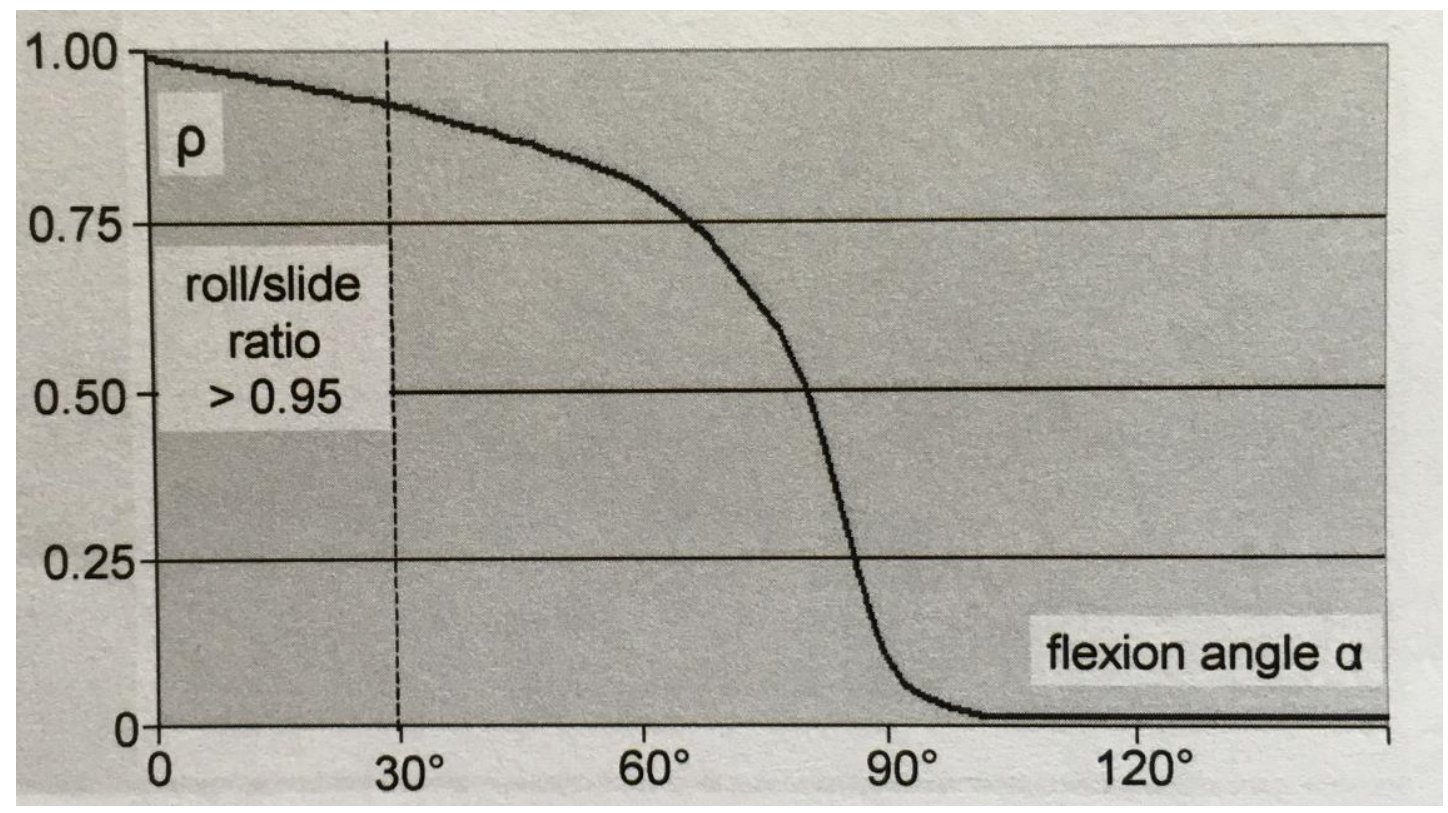

Abbildung 13: Berechnetes Roll-Gleitverhalten des AEQOUS-Knies im medialen Kompartiment nach Nägerl et al. (2008). Der Abdruck erfolgt mit freundlicher Genehmigung von Prof. H. Nägerl.

Nach diesen Ausführungen soll im Folgenden die Biomechanik des Kniegelenks detailliert betrachtet werden.

\subsection{Biomechanik des Tibiofemoralgelenks}

\subsubsection{Klassifikation von Gelenken}

Neben der Einteilung der Gelenke nach geometrischen Figuren, die eine Simplifizierung der natürlichen Gelenke darstellt, gibt es eine morphologische Einteilung nach der Verbindungsart in Diarthrosen und Synarthrosen, die im Folgenden dargestellt wird.

Diarthrosen sind echte Gelenke, die sich durch eine diskontinuierliche Verbindung zweier Knochen mit Gelenkspalt auszeichnen. Die artikulierenden Oberflächen sind zueinander inkongruent, wodurch zwangsläufig die Ausbildung eines Gelenkspaltes resultiert.

Dieser Gelenkspalt ist mit Synovia gefüllt (Fanghänel et al. 2003). Die Synovialflüssigkeit ermöglicht Nutrition und Lubrikation des Knorpels (Fanghänel et al. 2003; Schünke 2000).

Gelenke müssen in der Lage sein aneinandergrenzende Skelettteile miteinander zu verbinden und eine Kraftübertragung zu gewährleisten. Die verbundenen Skelettteile 
müssen sich dabei gegeneinander bewegen lassen (Kinematik), aber auch stabil miteinander verbunden sein (Statik) (Fanghänel et al. 2003).

Die vollständige kinematische Funktionsfähigkeit können Diarthrosen nur bei Kraftschluss erreichen (Kubein-Meesenburg et al. 1993). Dies wird im Kniegelenk über Druckkräfte der begleitenden Muskulatur und das Körpergewicht erreicht, worüber die notwendige Kontaktfläche für Bewegungen wie Gleiten und Rollen hergestellt wird.

Diarthrosen können nur Normalkräfte aufnehmen und transponieren. Im Gegensatz dazu können Synarthrosen mit ihrer kontinuierlichen Verbindungsmatrix Scherkräfte übernehmen und weiterleiten (Kubein-Meesenburg et al. 1993).

Synarthrosen (unechte Gelenke ohne Gelenkhöhle) sind dadurch gekennzeichnet, dass eine kontinuierliche Verbindung von zwei Knochen durch Bindegewebe (Syndesmosen), Knorpelsubstanz (Synchondrose) oder Verknöcherung von Knorpelsubstanz (Synostose) realisiert wird (Fanghänel et al. 2003). Auf diese Weise finden sich Verbindungen von geringer Beweglichkeit bis hin $\mathrm{zu}$ fast starren Kombinationen (Schünke 2000; Fanghänel et al. 2003).

\subsubsection{Funktionsmodell des Kniegelenks}

Das Kniegelenk ermöglicht durch seine zwei Funktionen, die Kinematik und die Statik, das Gehen, das aufrechte Stehen sowie die Möglichkeit zum Beispiel in der Hocke $\mathrm{zu}$ verharren. Das heißt, die gelenkige Verbindung von Femur und Tibia muss zum einen in der Lage sein den Gangzyklus, das heißt die Relativbewegung der beiden verbundenen Körperteile zueinander, zu ermöglichen, und zum anderen eine statische Position zu halten. Das Muskelsystem, das das Kniegelenk beeinflusst, hat sechs Freiheitsgrade und kann dadurch eine Position des Kniegelenks durch Ansteuerung unterschiedlicher Muskeln beeinflussen. Ändert sich hierbei vor allem die Richtung der Kraftwirkungslinie, kann eine stabile Position des Gelenks instabil werden (Aufstehen aus der Hocke).

Das Tibiofemoralgelenk kann aktiv bis etwa $150^{\circ}$ gestreckt werden (Extension), dann folgt noch eine Schlussrotation in der Endphase der Streckung, die 5-10 ${ }^{\circ}$ umfasst. Diese Position entspricht der in der anatomischen Literatur gängigen Bezeichnung $\mathrm{O}^{\circ}$-Position. Bei der Flexion können aktiv Winkelbereiche bis $120^{\circ}$ erreicht werden und passiv bis $\mathrm{zu} 160^{\circ}$. Die Flexion und Extension nehmen den größten 
Bewegungsumfang des Kniegelenks ein, und die Bewegung erfolgt praktisch in einer Ebene. Daher wird die Sagittalebene als Hauptfunktionsebene des Tibiofemoralgelenks betrachtet.

Die Funktion des Tibiofemoralgelenks wird durch die Gestaltung der artikulierenden Knochenoberflächen determiniert:

1. Inkongruenz der Gelenkflächen

Das mediale Kompartiment der Tibia ist konkav, das laterale Kompartiment konvex. Da der Krümmungsradius des konvexen femoralen medialen Anteils kleiner ist als der der konkaven tibialen Fläche, kommt es zur Inkongruenz der Gelenkflächen zueinander (Vergleiche Abbildung 14).

Im lateralen Kompartiment artikuliert die konvexe tibiale Kondyle mit der konvexen femoralen Kondyle (Abbildung 15).
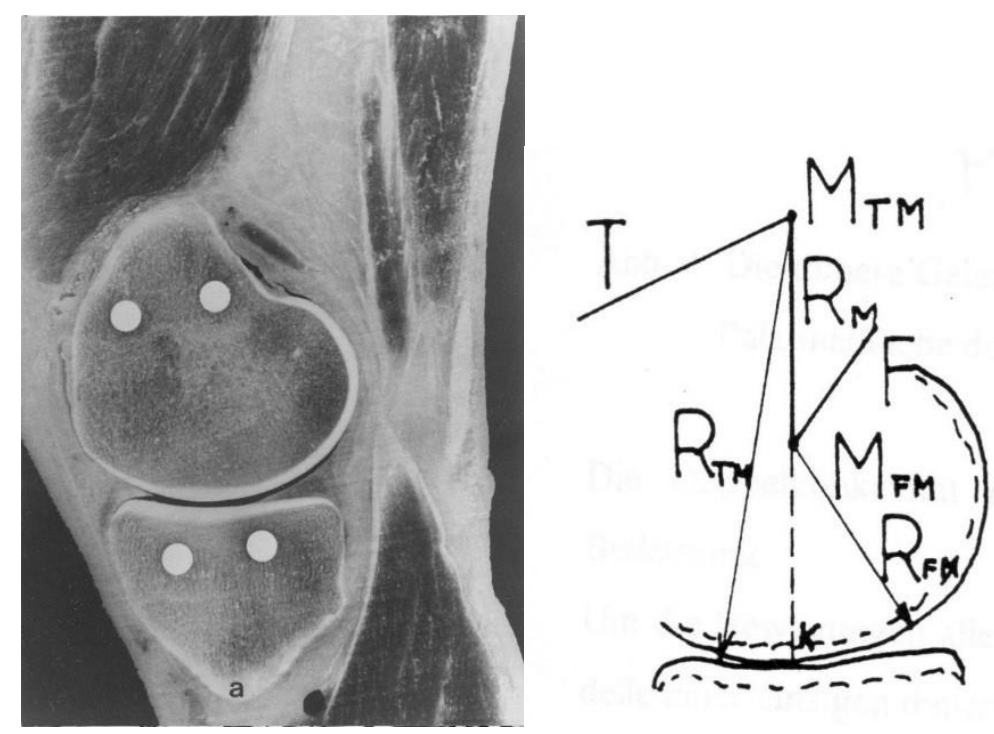

Abbildung 14: Mediales Kompartiment (Nägerl et al. 1993). Der Abdruck erfolgt mit freundlicher Genehmigung von Prof. H. Nägerl. 


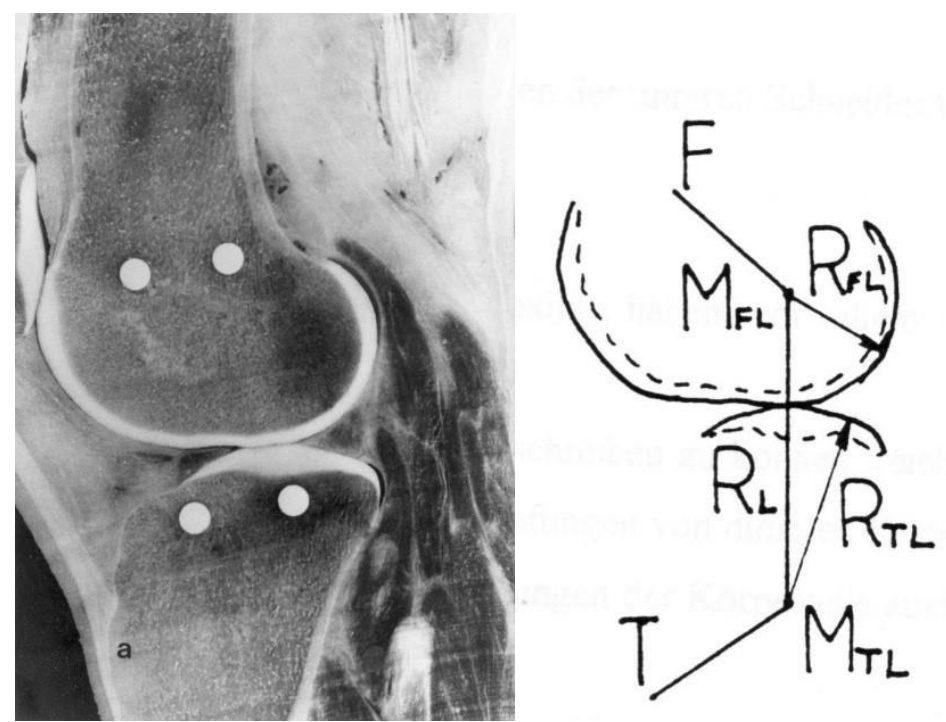

Abbildung 15: Laterales Kompartiment (Nägerl et al. 1993). Der Abdruck erfolgt mit freundlicher Genehmigung von Prof. H. Nägerl.

$F=$ Femur $;$ T Tibia $; M_{F L}=$ lateraler Kurvaturenmittelpunkt des Femurs, $M_{T L}=$ lateraler Kurvaturenmittelpunkt der Tibia, $R_{F L}=$ Radius der lateralen Kurvatur des Femurs, $R_{T L}=$ Radius der lateralen Kurvatur der Tibia, $M_{F M}=$ medialer Kurvaturenmittelpunkt des Femurs, $M_{T M}=$ medialer Kurvaturenmittelpunkt der Tibia, $R_{F M}=$ Radius der medialen Kurvatur des Femurs, $R_{T M}=$ Radius der medialen Kurvatur der Tibia, $R_{L}, R_{M}=$ Gestänge der dimeren Kette.

Im medialen Bereich liegt der tibiale Krümmungsmittelpunkt oberhalb des femoralen Krümmungsmittelpunktes (Abbildung 14), im lateralen jedoch unterhalb der femoralen Krümmungsmittelpunkte. Die mit den Krümmungsmittelpunkten $\mathrm{M}_{\mathrm{TM}}$ und $\mathrm{M}_{\mathrm{TL}}$ gegebenen Rotationsachsen fallen also nicht zusammen. Auch die entsprechenden femoralen Achsen $\mathrm{M}_{\mathrm{FM}}$ und $\mathrm{M}_{\mathrm{FL}}$ fallen nicht zusammen. Eine gemeinsame femorale Kondylenachse, wie sie in der Literatur in der Regel angenommen wird, gibt es demnach nicht. Wenn es sie gäbe, wäre das Knie mechanisch ein einfaches technisches Scharnier.

Der mediale femorale Kurvaturenmittelpunkt $\mathrm{M}_{\mathrm{FM}}$ ist gegenüber dem lateralen $\mathrm{M}_{\mathrm{FL}}$ um einige Millimeter nach anterior versetzt (Nägerl et al. 1993; Fiedler et al. 2011). Dadurch kommt das Viergelenk in der Extensionsstellung in der Sagittalebene während des menschlichen Ganges zustande.

Die Krümmungsmittelpunkte $\mathrm{M}_{\mathrm{FM}}$ und $\mathrm{M}_{\mathrm{FL}}$ sowie $\mathrm{M}_{\mathrm{TM}}$ und $\mathrm{M}_{\mathrm{TL}}$ haben einen konstanten Abstand, solange die Kontaktlinien durch die Kreise angenähert werden können. Damit können aufgrund der definierten Abstände die dimeren Ketten des lateralen und medialen Kompartiments zu einem Viergelenk aus vier Einzelgelenken gekoppelt werden (Nägerl et al. 1993). 
Im Falle des Kraftschlusses stellt das laterale Kompartiment also eine drucklabile dimere Gelenkkette dar, das mediale Kompartiment dagegen eine druckstabile überschlagene dimere Gelenkkette (Abbildung 14). Durch die kompressive Belastung des Gelenks, das heißt unter der Voraussetzung des Kraftschlusses, kann nun über Muskelkräfte sowohl ein mechanisch labiler als auch ein instabiler Gleichgewichtszustand hergestellt werden, wie es im nächsten Abschnitt näher erläutert wird.

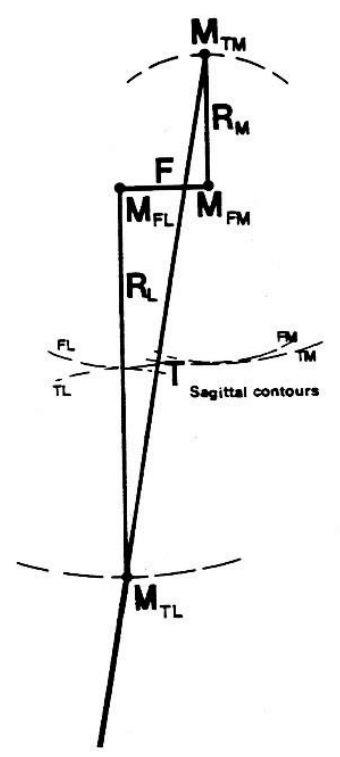

Abbildung 16: Dimere Ketten des Viergelenks (Nägerl et al. 1993). Der Abdruck erfolgt mit freundlicher Genehmigung von Prof. H. Nägerl.

\section{Gleichgewichtszustände}

Ein Körper befindet sich im Gleichgewicht, wenn die Summe aller einwirkenden Kräfte und die Summe aller einwirkenden Drehmomente Null ist:

$$
\begin{aligned}
& \sum F i=0 \\
& \sum T i=0
\end{aligned}
$$

Die Inkongruenz der artikulierenden Flächen führt dazu, dass eine Rotation nicht wie bei einem technischen Scharniergelenk um eine ortsfeste Achse verläuft. Stattdessen ist jeder Bewegungsvorgang des Gelenks die Aufeinanderfolge von differentiellen Einzelrotationen um die momentane Rotationsachse, deren Lage durch die Rotation um die Krümmungsmittelpunkte der Gelenkflächen ( $\mathrm{M}_{\mathrm{FL}}, \mathrm{M}_{\mathrm{FM}}$; $\mathrm{M}_{\mathrm{TL}}, \mathrm{M}_{\mathrm{TM}}$ ) zwangläu- 
fig hergestellt wird. Die momentane Rotationsachse eines Viergelenks, wie in Abbildung 17 dargestellt, kann keine ortsfeste Gerade wie eine Scharnierachse im raumfesten System sein, sondern sie wandert mit der jeweiligen Stellung des Viergelenks, entsprechend dem Flexionswinkel. Hierbei handelt es sich in der Hauptfunktionsrichtung des Tibiofemoralgelenks um eine ebene Bewegung.

In der Hauptfunktionsrichtung ergeben sich so die einzelnen momentanen Drehachsen. Diese bilden die Momentanpolkurve, auch Rastpolkurve oder helical axis genannt (Abbildung 17) (Blaha et al. 2003; Mansour et al. 2004; van den Bogert et al. 2008).

Für die Beurteilung eines Gleichgewichtzustandes ist die Rastpolkurve sowie die Kraftwirkungslinie entscheidend. Wird aus einem Gleichgewichtszustand mit isotonischer Kraft ausgelenkt, so unterscheidet man drei qualitative Gleichgewichtszustände: labil, stabil und indifferent.

Abbildung 17 demonstriert die Gleichgewichtszustände eines Viergelenks unter isotonischer Kraft:

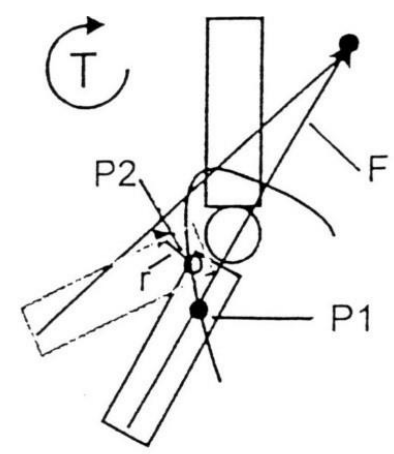

a) labiles Glgw.

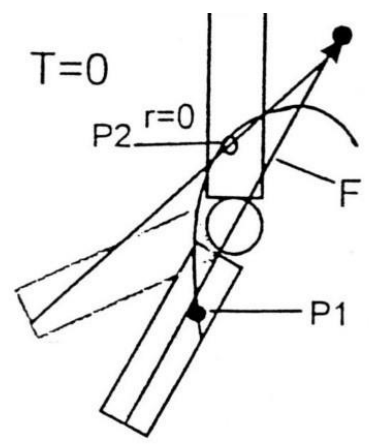

b) indifferentes Glgw.

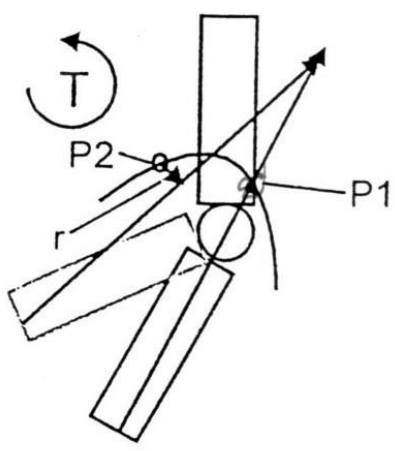

c) stabiles Glgw.

Abbildung 17: Gleichgewichtszustände eines Viergelenks unter isotonischer Kraft (Mackeben 1997). Der Abdruck erfolgt mit freundlicher Genehmigung von Dipl.-Phys. S. Mackeben.

$F=$ Kraft P1 = Punkt auf Rastpolkurve im Gleichgewicht P2 = Punkt auf Rastpolkurve nach Auslenkung

Ein labiles Gleichgewicht ist hergestellt, wenn bei einer Auslenkung der momentane Drehpol hinter der Kraftwirkungslinie zurückbleibt. Auf diese Art und Weise wird ein wegtreibendes Drehmoment erzeugt (Vergleiche Abbildung 17a).

Für ein stabiles Gleichgewicht hingegen ist ein rücktreibendes Drehmoment notwendig. Dieses wird erzeugt, wenn bei einer Auslenkung die Kraftwirkungslinie hinter dem momentanen Drehpol zurückbleibt. Auf diese Weise kann sich ein Viergelenk 
selbst stabilisieren (Vergleiche Abbildung 17c).

Ein indifferentes Gleichgewicht liegt vor, wenn die Kraftwirkungslinie nach der Auslenkung wieder durch den momentanen Drehpol verläuft (Vergleiche Abbildung 17b). Es wird in diesem Fall kein Drehmoment erzeugt, der Auslenkungszustand wird folglich beibehalten.

Die Rastpolkurve wird durch die Gestalt der artikulierenden Oberflächen bestimmt. Die Änderung des Gleichgewichtzustandes erfolgt durch die veränderbare Kraftwirkungslinie.

\section{Freiheitsgrade}

Die Flexion/Extension stellt den Hauptfreiheitsgrad des Tibiofemoralgelenks mit dem größten Bewegungsumfang dar. Er ist von der Muskulatur bedienbar und einstellbar.

Die sogenannten drei kleinen Freiheitsgrade des Tibiofemoralgelenks - die zwei Rotationen um die Flächennormalen in den beiden Kontaktpunkten und die Ab-/Adduktion - sind muskulär nur unwillkürlich beeinflussbar. Sie werden durch die kompressive Gelenkkraft in einem stabilen Zustand gehalten, da sich die Kontaktpunkte auf den Abhängen des Tuberculum intercondylare befinden, wie es technisch bei einer Laufkatze zu finden ist. Untersuchungen unserer und anderer Arbeitsgruppen belegen, dass die spezielle Mechanik des Kniegelenks auf die Geometrie der Tibia-/FemurFlächen und deren Anordnung zueinander zurückzuführen ist (Nägerl et al. 1993; Martelli et al. 2002; Fiedler et al. 2011; Hamai et al. 2013).

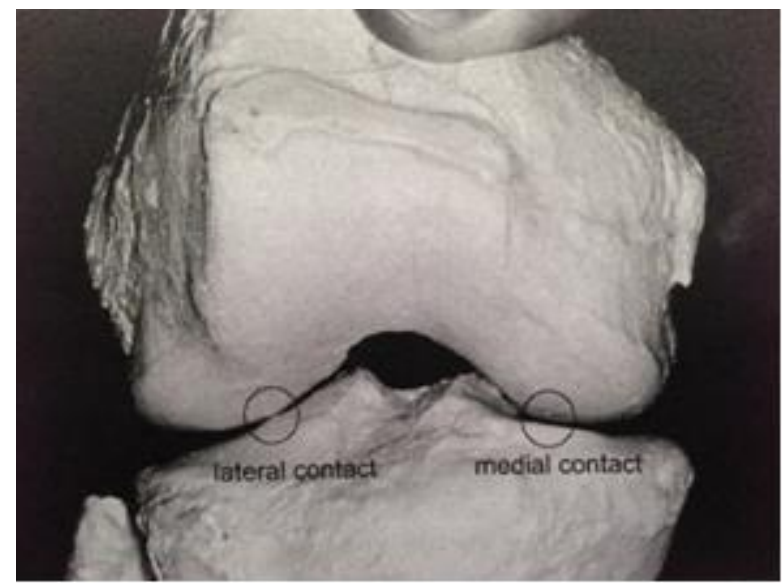

Abbildung 18: Frontale Ansicht des Tibiofemoralgelenks mit Kontaktpunkten (Fiedler et. al 2011). Der Abdruck erfolgt mit freundlicher Genehmigung von Dr. C. Fiedler.

Die kinematischen Eigenschaften des Viergelenks (Vergleiche Seite 21) des Tibiofemoralgelenks ergeben sich unter anderem aus den Verhältnissen und Längen 
der Getriebeglieder. Dies soll im Folgenden erläutert werden.

4. Kinematik des Kniegelenks

Neben der primär durchgeführten Bewegung des Kniegelenks, der Extension-Flexion in der Sagittalebene (Hauptfreiheitsgrad), betrachten wir noch die so genannten „kleinen“ oder Neben-Freiheitsgrade (Knösel 2003), Bewegungen der Ab- und Adduktion sowie der axialen Rotation (Pronation/Supination) und die Translation in anteriorerposteriorer Richtung.

Diese kinematischen Funktionen dürfen nicht durch eine unzulässige Idealisierung des Tibiofemoralgelenks zu einem technischen Scharniergelenk vernachlässigt werden. Sowohl die Innenrotation des Femurs und die Außenrotation der Tibia bei steigender Extension und die Innen- sowie Außenrotation der Tibia bei Flexion und Pronation/Supination als passive herbeigeführte Bewegungen tragen zur Stabilisierung des Knies insbesondere unter Last wesentlich bei (Meyer 1873; Hill et al. 2000; Iwaki et al. 2000; Markolf et al. 2014; Nägerl et al.1993).

Die Lage der Kontaktpunkte des Femurs und der Tibia in der Frontalen auf den Abhängen des Tuberculum intercondylare führt $\mathrm{zu}$ einer Selbststabilisierung des Tibiofemoralgelenks bei externen Krafteinflüssen auf das Gelenk, wie sie zum Beispiel beim Gehen auf unebenem Gelände zwangsläufig entstehen.

Das Vorliegen eines Kraftschlusses im Tibiofemoralgelenk ist Voraussetzung für das Rollen und Gleiten im Gelenk. Dies wird im humanen Tibiofemoralgelenk durch die Gewichtskraft, die Muskelkräfte und auch durch Bandkräfte realisiert.

Ferner wird die Qualität des Kraftschlusses durch die in den Menisci und den Kreuzbändern vorhandenen Rezeptoren registriert und kontrolliert (Zimny et al. 1988; Nägerl et al. 1993; Gray 1999).

Nägerl et al. (2004) ermittelten im natürlichen Kniegelenk, dass zwischen $5^{\circ}$ und $25^{\circ}$ der Extension der Anteil des Rollens groß und das Problem der Reibung deshalb gering ist. Dieser Sachverhalt wird auch in Lehrbüchern qualitativ dargestellt (Fanghänel et al. 2003, Kapandji 2009).

Betrachtet man den Gangzyklus, so wird die Sinnhaftigkeit der tibiofemoralen Geometrie deutlich, und es wird klar, dass die Inkongruenz keineswegs ein Versehen der Natur ist. Die Möglichkeit, dass die Tibia in der Anfangsphase der Flexion abrollen kann, ist für die Langlebigkeit der artikulierenden Flächen entscheidend, da in dieser 
Phase die Gewichtsbelastung am höchsten ist. Das Abrollen der Tibia unter Last sorgt dafür, den Verschleiß der artikulierenden Flächen möglichst gering zu halten.

Mit steigenden Flexionswinkeln hin zur Schwungphase des Ganges, ist das Tibiofemoralgelenk des Spielbeins weniger belastet, so dass eine Entlastung durch Abrollen nicht mehr notwendig ist (Abbildung 12).

In der Anfangsphase des Gehens, in der die mit Abstand größte Gewichtsbelastung auftritt, ist das Abrollen besonders wichtig, da das Bein über den Bodenkontakt mit dem Gewicht des restlichen Körpers belastet wird. Im medialen Kompartiment findet ab etwa $10^{\circ}$ der Vorgang des Roll-Gleitens statt (Thomsen 2005).

Die Inkongruenz der artikulierenden Flächen bewirkt im lateralen Kompartiment ab circa $35^{\circ}$ einen abermaligen Anstieg der Rollbewegung. Dies ist als Schutzmechanismus gegen ein Gleiten des Femurs von der Tibia zu sehen.

Vor diesem physikalischen Hintergrund wird im nächsten Abschnitt ein Überblick über die Grundlagen des vollständigen Kniegelenkersatzes gegeben. Dabei wird auf die Indikation zum Endoprothesenersatz und auf die in der vorliegenden Arbeit vermessenen Endoprothesen eingegangen.

\section{$2.4 \quad$ Knie-Endoprothesen}

\subsubsection{Ziel und Indikationen}

Das Ziel eines jeden Kniegelenkersatzes ist es, eine durch verschiedene Ursachen eingetretene schmerzhafte Malfunktion des natürlichen Kniegelenks zu beheben und die natürliche Funktion so gut wie möglich wiederherzustellen, um somit dem Patienten ein schmerzfreies Leben ermöglichen zu können.

Anforderungen an die Implantate sind (Witzel 2000):

- eine hohe Belastungsstabilität

- keine Relativbewegung zwischen Knochenlager und Implantat

- Standhalten gegen die hohe mechanische Belastung, das heißt gute Alterungsbeständigkeit

- biologische Implantatintegration

- Erhalt der physiologischen Spannungsverteilung im Knochen. 
Die Indikation für Kniegelenksendoprothesen ist primär die Gonarthose, in den meisten Fällen der chronische Gelenkschmerz bei einer Gonarthrose, welcher durch eine konservative Therapie nicht mehr zu beherrschen ist. Die Gonarthrose inklusive Achsfehlstellungen, Knochenverlust und Bandinstabilitäten müssen klinisch und radiologisch abgeklärt werden (AWMF-Leitlinie 2011).

Ursachen können eine Osteoarthrose sowie Frakturen oder eine rheumatische Arthritis sein. Folgen einer Osteoarthrose sind Funktionseinschränkungen durch verminderte Propriozeption, Schmerzen und Muskelatrophie und damit einhergehend Stabilitätsverlust und erhöhte Frakturanfälligkeit. Charakteristisch sind vor allem Ruheschmerz sowie Schmerzen in der Nacht, die neben dem Bewegungsschmerz auftreten.

Funktionelle Einschränkungen im fortgeschrittenen Stadium einer Gonarthrose, die zu einer deutlichen Einschränkung der Mobilität führen, machen eine Operation notwen$\operatorname{dig}$ (Imhof 2005).

Das Ziel der Knieendoprothetik besteht primär darin, den Patienten von seinen chronischen Schmerzen zu befreien und ihm dabei ein hohes Maß an Beweglichkeit und „Stabilität“ wiederzugeben, das heißt seine Lebensqualität anzuheben und ihm eine Reintegration in Alltags- und Berufsleben zu ermöglichen. Ein wichtiges Ziel ist dabei die Langzeitstabilität (AWMF-Leitlinie; Jerosch und Floren 2000; Imhof 2005).

\subsubsection{AEQUOS-Knie}

AEQUOS-Knie (AEQUOS Endoprothetik GmbH, Gräfelfing, Deutschland) ist ein bikondylärer Oberflächenersatz für das Kniegelenk. Die AEQUOS-Endoprothese war als eine der wenigen auf dem Markt befindlichen Endoprothesen der natürlichen Gelenkmorphologie nachempfunden: Sie kann daher funktionell die natürliche RollGleitbewegung, die axiale Rotation, die Ad-/Abduktion und die Überstreckungshemmung des Kniegelenks nachvollziehen.

Das AEQUOS-Knie ermöglicht ein Rollen bis zu einer Beugung von circa $30^{\circ}$, also genau dann, wenn die hohe äußere Belastung in der Standphase auftritt. Dadurch wird das Auftreten von Haft- und Gleitreibung und damit eine Scherbelastung des Inlays aus Polyethylen vermieden (Abbildung 21).

Das Material der Femurkomponente und der Tibiaplatte ist eine hochfeste Cobalt- 
Chrom-Molybdän-Legierung (CoCrMo), der zentrale Zapfen und das in die Platte eingesetzte Inlay bestehen aus ultrahochmolekularem Polyethylen (UHMWPE). Femurund Tibiakomponente sind in jeweils fünf verschiedenen Größen erhältlich. Das Polyethylen-Inlay wird durch eine patentierte Methode auf der Metallbasis fixiert (Abbildung 19).

Das Inlay wird präoperativ gekühlt und muss gekühlt in die passende metallische Basis der Tibiakomponente eingebracht werden. Erwärmt sich nun das Inlay, dehnt es sich in Folge dessen aus, und es wird eine Presspassung in der Basis erreicht. Hierdurch wird der Gefahr von Abrieb zwischen Inlay und Metallbasis durch Mikrobewegungen wirkungsvoll begegnet.

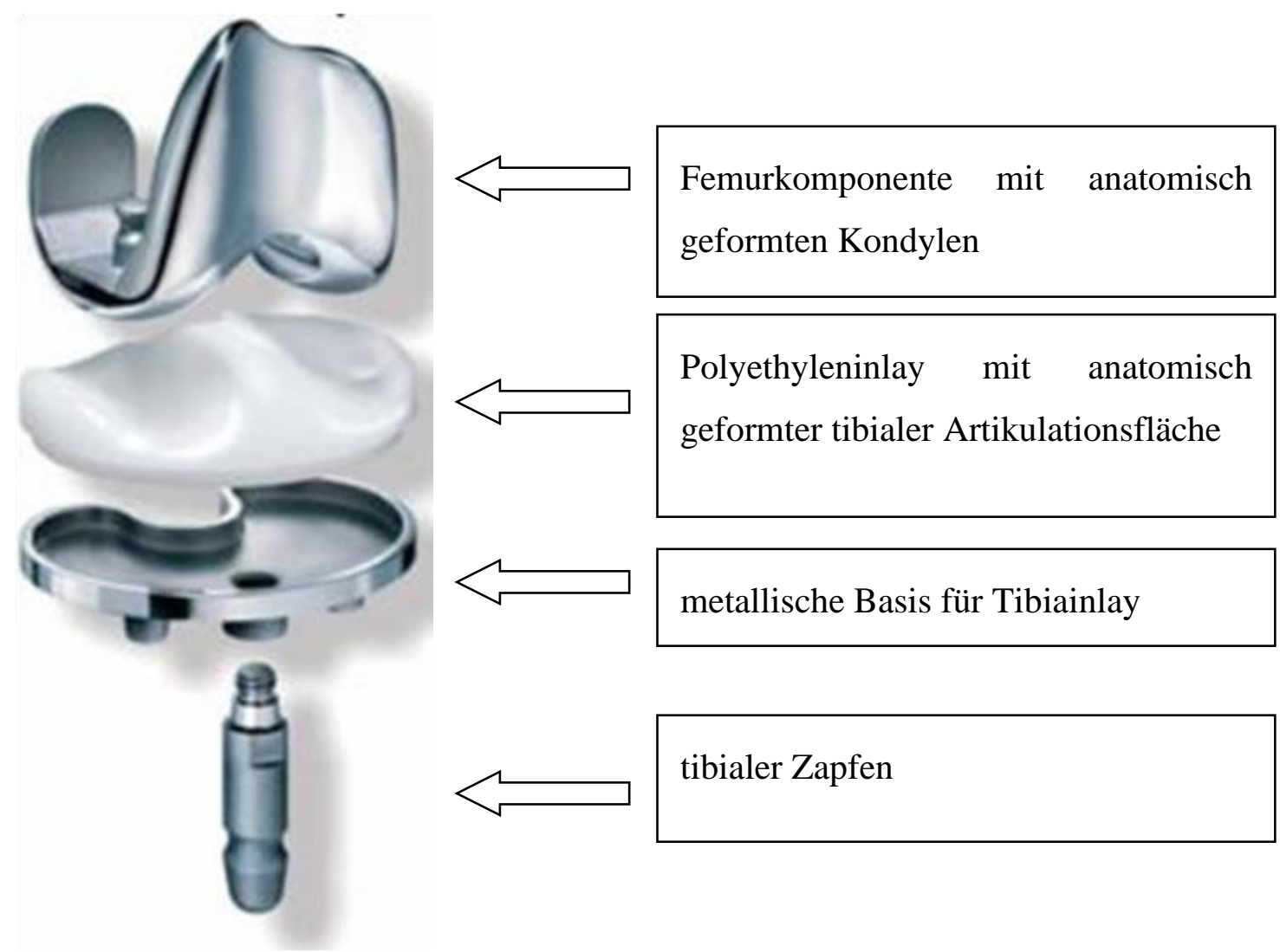

Abbildung 19: AEQUOS-Knie-Endoprothese (http://www.neu.uni-bayreuth.de/de/Uni_Bayreuth/Fakultaeten/6_FAN/Metallische_Werkstoffe_neu_unfertig/de/research/polymer/index.html; Zugriff am 09.04.2018).

Die medialen und lateralen Gelenkflächen des Femurs besitzen in Funktionsrichtung einen annährend gleichen Krümmungsradius.

Die Rotationsmittelpunkte der Achsen sind aber in sagittaler Richtung etwas versetzt: 
Der Rotationsmittelpunkt der medialen Gelenkfläche liegt um einige Millimeter weiter vorn (Asymmetrie) (Abbildung 20) (Frosch et al. 2009; Nägerl et al.1993, Nägerl et al. 2008). Wie in Abbildung 21 veranschaulicht, kann auf diese Weise die Mechanik des natürlichen Kniegelenks nachempfunden werden und es liegt ein überwiegendes Rollen im Winkelbereich von 0-30 ${ }^{\circ}$ vor (Frosch et al. 2009). Bei Kraftschluss liegt im AEQUOS-Knie ein Zwanglauf bedingt durch die Artikulationsflächen vor. Es gibt in Flexion/Extension einen Freiheitsgrad. Die momentane Drehachse liegt bei kleinen Flexionswinkeln nahe den beiden Kontaktpunkten von Femur und Tibia, so dass Rollen vorliegt (Vergleiche Abbildung 13). Steigt der Flexionswinkel entsprechend dem Gangzyklus, wandert die momentane Drehachse zum Mittelpunkt der femoralen Kondylen. Dies bedingt ein Gleiten in der Schwungphase (Nägerl et al. 2008, Frosch et al. 2009).

Des Weiteren zeigt das AEQUOS-Knie wie das natürliche Tibiofemoralgelenk bedingt durch die Positionierung der Kontaktpunkte auf den tibialen Abhängen im frontalen Schnitt einen Bewegungsspielraum der Prothese in der axialen Rotation und der Varus/Valgus Translation, der für die Selbststabilisierung im Sinne einer Laufkatze vor allem bei äußerer Kraftwirkung sorgt. So unterliegen diese Freiheitsgrade bei kraftschlüssigem Gelenk einer Selbststabilisierung.
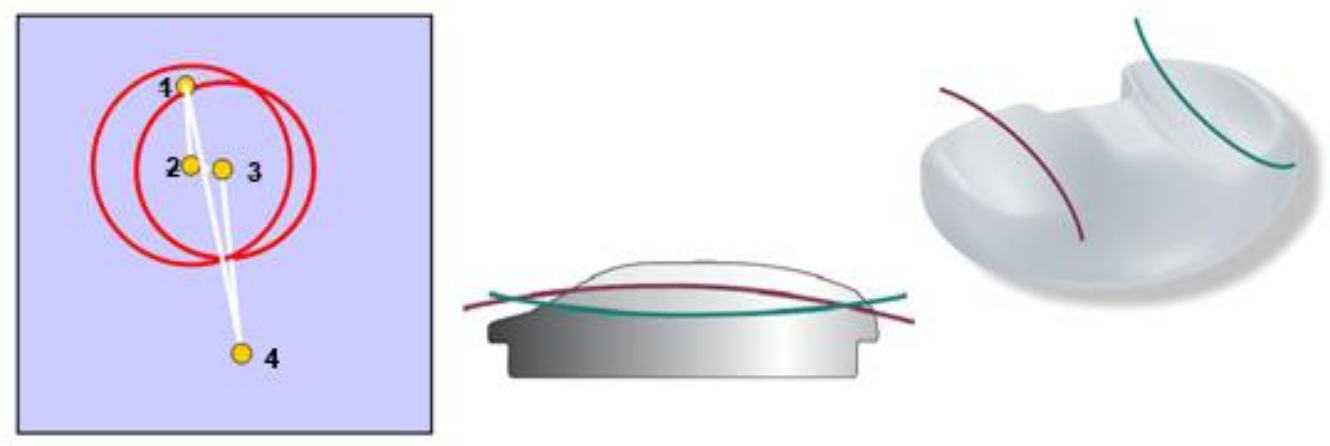

Abbildung 20: Konstruktion der Gelenkflächen des tibialen Inlays der AEQUOS-Endoprothese (http://www.aequos.de/de/arzt/reproduktion.htm; Zugriff am 11.04.2018).

$1=M_{T M}$ Mittelpunkt Tibia medial $2=M_{F M}$ Mittelpunkt Femur medial $3=M_{F L}$ Mittelpunkt Femur lateral $4=M_{T L}$ Mittelpunkt Tibia lateral 


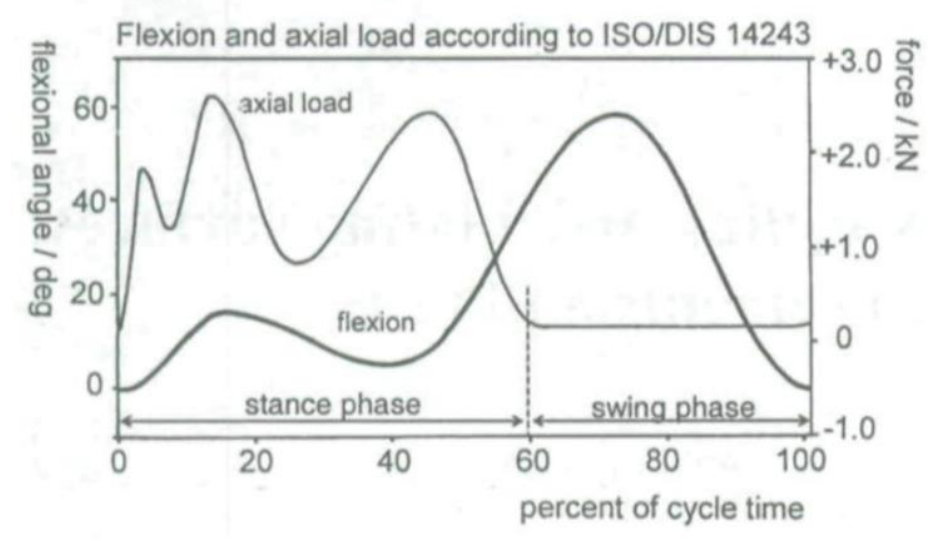

Abbildung 21: Gangzyklus mit axialer Belastung des Kniegelenks (Nägerl et al. 2008). Der Abdruck erfolgt mit freundlicher Genehmigung von Herrn Prof. H. Nägerl.

\subsubsection{Mebio Scan-Knie}

Im Vergleich wird das Mebio Scan-Knie untersucht, welches sich durch konvex-konkave symmetrische Oberflächengestaltung in beiden Gelenkkompartimenten auszeichnet.

Inkongruenzen entstehen, da die Radien der tibialen Konkavitäten deutlich größer sind als die Radien der femoralen Konvexitäten. Es gibt hier eine Femurachse und eine Tibiaachse. Funktionell stellt die Prothese eine überschlagene dimere Gelenkkette dar. Sie hat für die Flexion/Extension keinen Zwanglauf wie das natürliche Kniegelenk und in der Flexion/Extension zwei kinematische Freiheitsgrade. Sie kann um die Tibiaachse und/oder die Femurachse rotieren. Bei gleichsinniger Rotation liegt die momentane Drehachse zwischen Femur- und Tibiachse, demnach also immer oberhalb der Femurachse, so dass dann ein Rollen nicht möglich ist.

Die Mebio Scan-Knieendoprothese besteht aus denselben Materialien wie die AEQUOS-Endoprothese.

Das Mebio Scan-Knie wurde bis jetzt als eine angeblich dem natürlichen Knie nachgebildete Prothese vermarktet, aber es hat gegenüber dem AEQUOS-Knie den entscheidenden Unterschied, dass tibiale und femorale Kompartimente symmetrische Artikulationsflächen haben und aufgrund dieser Konstruktion das natürliche Knie nicht nachbilden können, da sie das konvexe laterale Plateau des natürlichen Knies ignoriert. 


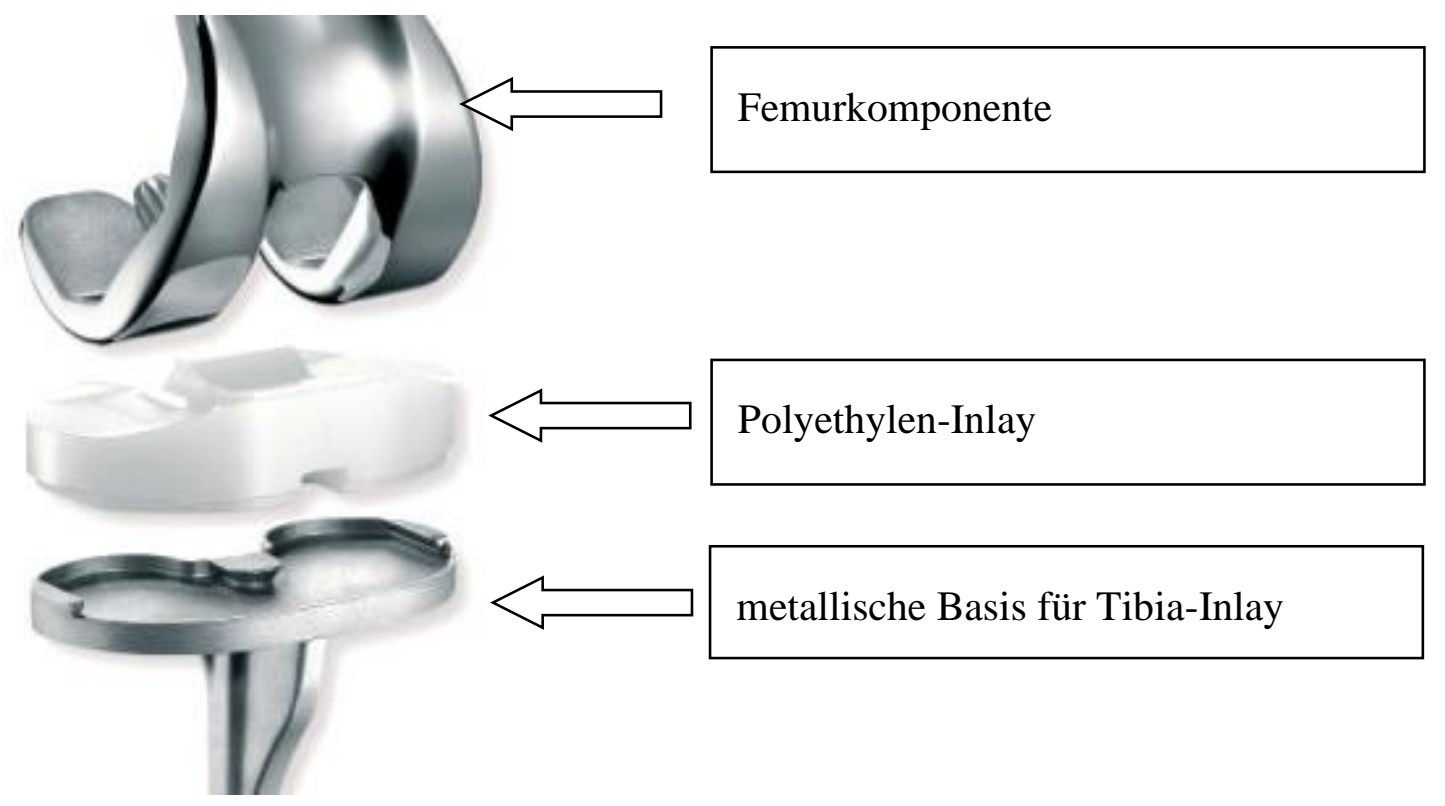

Abbildung 22: Aufbau des Mebio Scan-Knies.

(http://www.aap.de/de/Produkte/Endoprothetik/Knieendprothetik/Mebio_Scan/index.html; Zugriff am 04.02.2005). 


\section{$3 \quad$ Methodik}

\subsection{Die Idee des Messaufbaus}

Die Funktionsweise des menschlichen Tibiofemoralgelenks ist auf die morphologische Gestaltung seiner artikulierenden Flächen zurückzuführen (Nägerl 1990, Nägerl et al. 1993). Diese bedingt, wie in den vorherigen Kapiteln dargestellt, das für das Tibiofemoralgelenk charakteristische Roll-Gleitverhalten, seine Selbststabilisierung bei externem Krafteinfluss sowie seine Fähigkeit, qualitativ unterschiedliche Gleichgewichtseinstellungen einnehmen zu können. Endoprothesen sollten in möglichst weitem Spektrum diese Eigenschaften wiedergeben.

In der vorliegenden Arbeit wurde untersucht, wie das dem natürlichen Kniegelenk nachempfundene AEQUOS-Knie im Vergleich zur Mebio Scan-Prothese die unterschiedlichen Gleichgewichtsmodifikationen des Tibiofemoralgelenks darstellen kann. Die Eigenschaften des Tibiofemoralgelenks - bedingt durch die morphologische Gestaltung seiner artikulierenden Flächen - sind maßgeblich für die uns zur Verfügung stehenden Bewegungsmöglichkeiten unseres Kniegelenks wie Gehen und Stehen, Hocken und Aufstehen aus dem Sitzen verantwortlich. Für diese Bewegungen spielen die jeweiligen Gleichgewichtspositionen des Kniegelenks und ihre Qualität die entscheidende Rolle. Ein Kniegelenkersatz sollte in der Lage sein, diese natürlichen Funktionsmuster, wie es Nägerl et al. (1993) beschrieben, wiederzugeben.

Das AEQUOS-Knie und das Mebio Scan-Knie divergieren vor allem in der Gestaltung ihrer Artikulationsflächen.

So zeichnet sich das Mebio Scan-Knie durch Symmetrie aus, das heißt die Rotationsachsen im lateralen und femoralen Kompartiment fallen zusammen. Es hat somit zwei Freiheitsgrade in Flexion/Extension, da es eine dimere Kette darstellt (Nägerl et al. 2008). Das AEQUOS-Knie hingegen zeigt ein asymmetrisches Design: In der Sagittalen zeigt sich lateral das tibiale Plateau konvex und das mediale tibiale Plateau konkav. Wie diese Unterschiede die Gleichgewichtseinstellung der Endoprothesen beeinflussen, zeigt diese Arbeit auf.

Das Tibiofemoralgelenk besitzt vier Freiheitsgrade. Eine mögliche Stellung des Gelenks wird durch eine Vielzahl an Muskelkräften erzeugt: Musculus quadriceps femoris, Musculus popliteus, Musculus gastrocnemius und die Hamstring-Gruppe. Somit hat das muskuläre Kraftsystem sechs Freiheitsgrade. 
Demzufolge können in jeder Gelenkposition prinzipiell Gleichgewichte unterschiedlicher Qualität hergestellt werden.

Aufzuzeigen, wie sich die Gleichgewichtsverhältnisse in den beiden Prothesentypen darstellen, ist Ziel dieser Arbeit. Bei vorgegebenen Flexionswinkeln sollte dafür ein Gleichgewicht der Knieendoprothesen in dem vorliegenden Messaufbau hergestellt werden. Hierbei wurde das Femur fest im Messaufbau verankert und die Tibia kraftschlüssig mittels der simulierten Muskelkräfte positioniert. Aus dieser Gleichgewichtsposition heraus erfolgten Auslenkungen in Flexion- und Extensionsrichtung, und das auftretende Kraftsystem, das heißt die rücktreibenden oder wegtreibenden Kräfte und Drehmomente wurden gemessen. Diese Kräfte und Drehmomente wurden in Kurven graphisch dargestellt (Vergleiche Kapitel 4). Im selben vorgegebenen Flexionswinkel wurden unterschiedliche imitierte muskuläre Kraftsysteme durch Variation der erzeugten Gewichtskräfte sowohl auf das AEQUOS-Knie als auch auf das Mebio Scan-Knie aufgebracht, wobei immer eine Gleichgewichtsposition erzeugt wurde. Von diesen Gleichgewichtslagen aus erfolgten erneut die Auslenkungen in Flexion- und Extensionsrichtung, und die auftretenden Kraftsysteme wurden gemessen. Als Flexionswinkel wurden zwei Positionen gewählt: Zum einen $5^{\circ}$, welches dem Stand beziehungsweise dem Beginn des Ganges entspricht und zum anderen $90^{\circ}$, welches dem Kniewinkel beim Aufstehen aus der Hocke oder dem Sitzen entspricht. Durch diesen Versuchsaufbau soll die Veränderbarkeit der Qualität der Gleichgewichte demonstriert werden. Diese Veränderbarkeit ermöglicht erst das Hocken, welches einem stabilen Gleichgewicht entspricht, und das Aufstehen aus einer Hockposition, welches ein labiles Gleichgewicht voraussetzt, soll es ohne Hilfe durchgeführt werden.

Für die Untersuchung von Viergelenken des Tibiofemoralgelenks wurde 1997 von Mackeben eine Apparatur entwickelt. Diese war dafür konzipiert, ein technisches Viergelenk, das mit dem Aufbau des menschlichen Tibiofemoralgelenks zu vergleichen ist, hinsichtlich seiner Stabilität der Gleichgewichte (instabil versus stabil) zu untersuchen. 2002 modifizierten erst Stühmer und ein Jahr später Knösel (2003) Mackebens Messapparatur, um frische Kniegelenke vom Hausschwein hinsichtlich der Qualität ihrer Gleichgewichtspositionen messen zu können.

Die vorliegende Arbeit stellt die Weiterentwicklung der Messapparatur dar, um die 
Qualität und Quantität von stabilen und labilen Gleichgewichtslagen von zwei unterschiedlich konzipierten menschlichen Knieendoprothesen zu bestimmen. In dem vorliegenden Versuchsaufbau werden zwei Knieendoprothesen in Gleichgewichtsstellungen für unterschiedliche Flexions-/Extensionsgrade durch Kraftsysteme, die dem das Kniegelenk beeinflussenden muskulären Apparat modellhaft nachgebildet sind, gebracht.

Zunächst soll die Entwicklung der Messapparatur von Mackeben über Stühmer zu Knösel dargestellt und anschließend die Anforderungen an einen Aufbau der Messapparatur für Knieendoprothesen und die daraus folgende vorgenommene Weiterentwicklung diskutiert werden. Dem folgt die Beschreibung des Versuchsaufbaus und seiner Durchführung im Detail.

\subsubsection{Entwicklung und Prinzip des Messaufbaus}

Das in der vorliegenden Arbeit angewandte Prinzip des Messaufbaus geht auf die Anordnung von Mackeben (1997) zurück, mit der Gleichgewichtszustände technischer Gelenke beurteilt werden konnten.

Mackeben benutzte ein - dem Viergelenkmodell des Tibiofemoralgelenks für Flexion/Extension entsprechendes - technisches Viergelenk. Zur Vereinfachung sollen hier die Bezeichnung Tibia und Femur verwendet werden, wie sie auch Mackeben benutzte (Mackeben 1997). Dabei entspricht das Femur dem Gestell und die Tibia der Koppel des Viergelenks. Die Bewegung des technischen Gelenks bei Mackeben erfolgt ausschließlich in einer Ebene. Die Messapparatur bei Mackeben genügte nur der Messung von ebenen Gelenken.

In einem raumfesten System, im vorliegenden Fall dem äußeren Gestell der Messapparatur, war das Femur fixiert. Die Tibia wurde über eine am Außengerüst fest installierte Kreisbogenschiene fixiert und darüber auch ausgelenkt, indem Auslenkungswinkel über Arretierschrauben festgelegt werden konnten. 


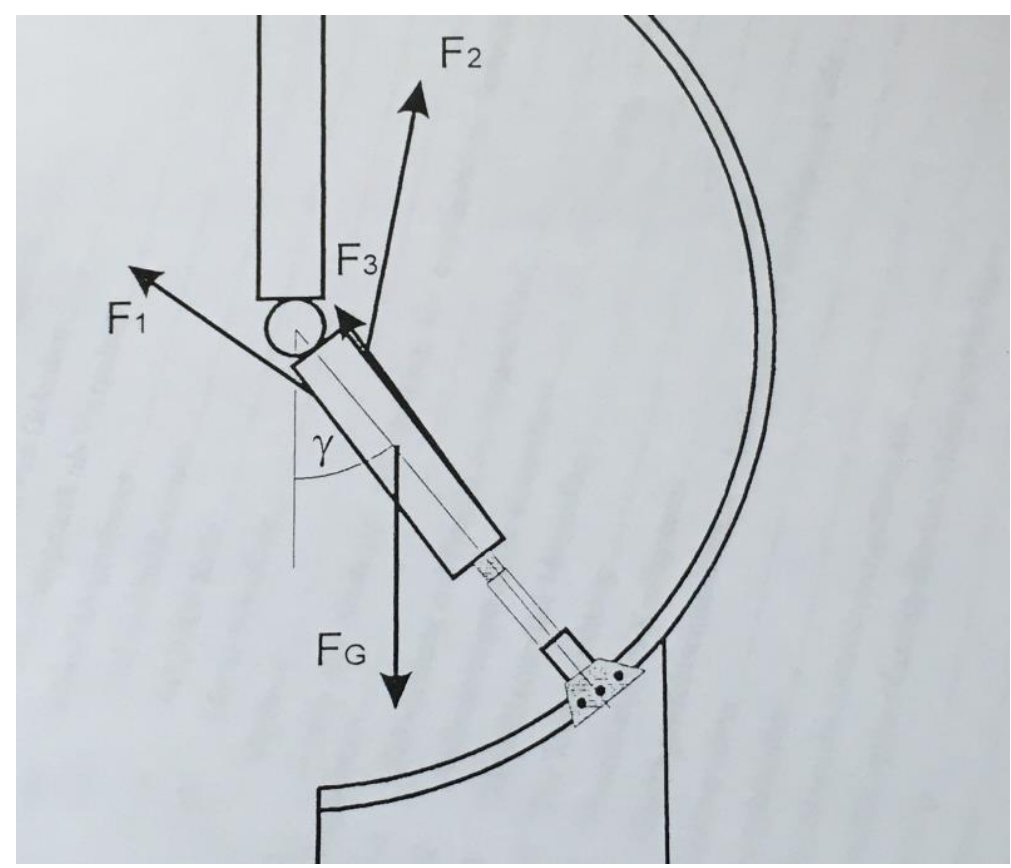

Abbildung 23: Skizze der Messapparatur und des Kraftsystems nach Mackeben (1997). Der Abdruck erfolgt mit freundlicher Genehmigung durch S. Mackeben.

F1: Musculus quadriceps F2: Hamstrings F3: Musculus gastrocnemius Fg: Gewichtskraft Tibia $\gamma$ : Flexionswinkel

Die Tibia wurde mit einem isotonischen Kraftsystem belastet, das der Muskulatur des Kniegelenks nachempfunden war. Auf den Musculus popliteus wurde verzichtet, da das Gelenk nur ebene Auslenkung zuließ. Für die Simulation der Muskelkräfte wurden gefüllte Wasserkanister verwendet. Die Seile, an denen diese befestigt waren, wurden in Gewindebohrungen, die sich an der Tibia befanden, verankert. Die Seile wurden über kugelgelagerte Umlenkrollen zu den Wasserbehältern geführt. Die einzelnen Seilkräfte wurden mittels eines Buckle-Transducers gemessen.

Eine Kraftmessdose war an der Tibia und Kreisbogenschiene fest arretiert, damit das auf die Tibia bei der Messung auftretenden Kraftsystem registriert werden konnte.

Stühmer (2002) war vor das Problem gestellt, die Apparatur so zu verändern, dass kein ebenes technisches Gelenk, sondern ein biologisches Tibiofemoralgelenk mit allen relevanten Freiheitsgraden vermessen werden konnte. Dabei stellte sich als Hauptproblem heraus, dass die Kreisbogenschiene bei Fixierung der Tibia eine Zwangsführung in der Tibiabewegung bedingte, die unnatürliche Kräfte im Tibiofemoralgelenk produzierte, weil die Schiene die kleineren Freiheitsgrade blockierte. Damit waren außer dem Hauptfreiheitsgrad der Extension/Flexion alle übrigen Freiheitsgrade durch diese Anordnung inaktiviert. 
Stühmer verwendete ebenfalls Wasserkanister für die Simulation der Muskelkräfte und fixierte die Tibia fest über die Kraftmessdose an der Kreisbogenschiene.

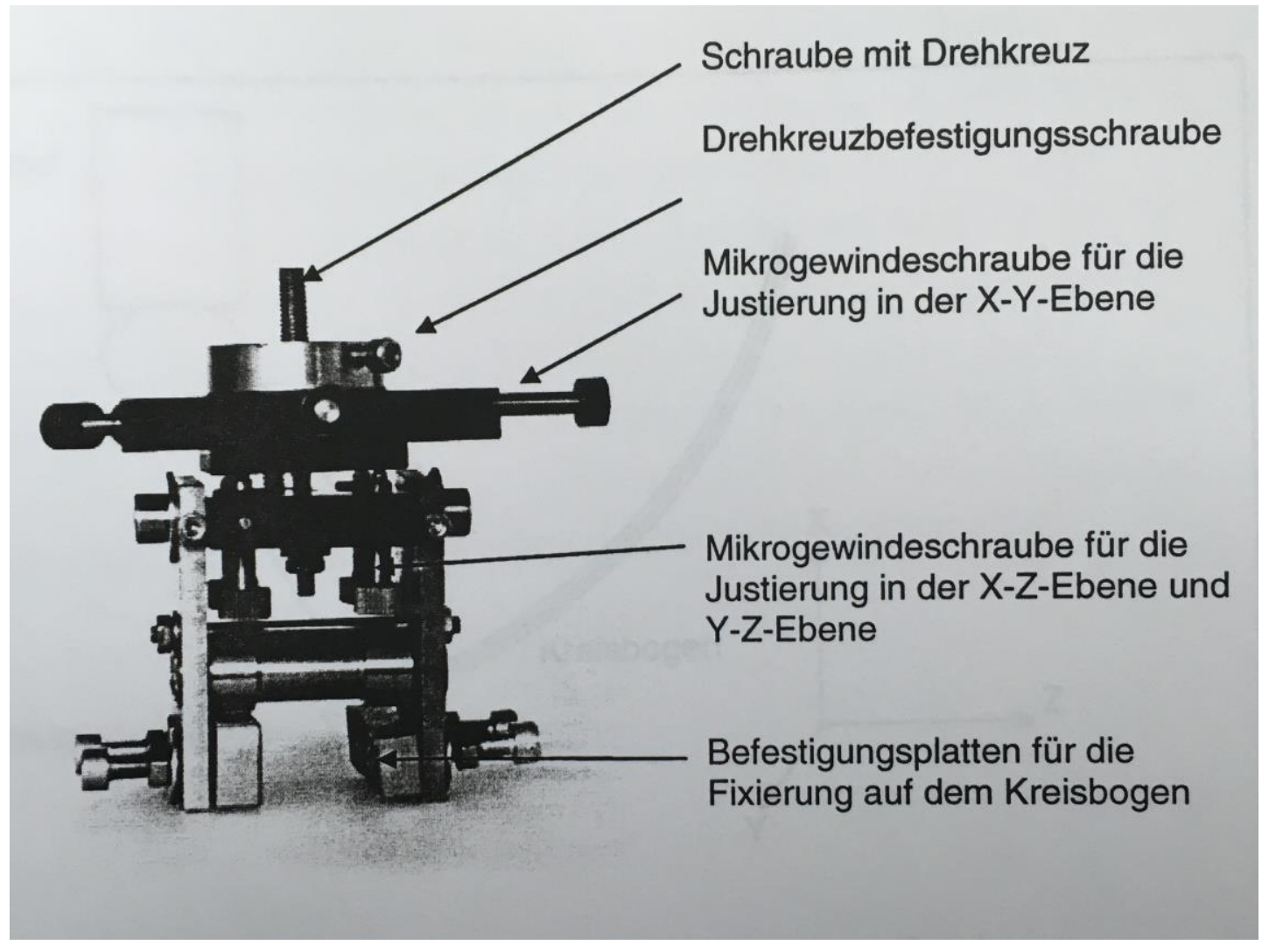

Abbildung 24: Verbindungsteil der Kraftmessdose mit dem Kreisbogen (Stühmer 2002). Der Abdruck erfolgt mit freundlicher Genehmigung von Dr. K. Stühmer.

Knösel (2003) versuchte die Zwangskräfte zu neutralisieren, die durch eine starre Befestigung der Tibia mit der Kreisbogenschiene hervorgerufen wurden, indem er die Tibia gelenkig mit der Kreisbogenschiene verband. Um Zwangskräfte zu vermeiden, installierte Knösel zwei Lager (Vergleiche Abbildung 27) für die Befestigung der Tibia sowohl mit der Kraftmessdose als auch mit der Kreisbogenschiene (Knösel 2003). Dies sollte dazu führen, dass bei der Auslenkung der zu untersuchenden Kniegelenke des Hausschweins außer dem Hauptfreiheitsgrad in der Flexion-/Extensionsbewegung auch die kleinen Freiheitsgrade verfügbar waren.

Knösel beschreibt, dass die Installation der Lager Zwangskräfte innerhalb des Kniegelenks bei der Auslenkung verhindere.

Im Rahmen von Vorversuchen für die Messungen dieser Arbeit, die notwendig waren um die notwendigen Modifikationen des vorhandenen Messsystems zu erarbeiten, 
zeigte sich, dass durch diese Lager trotzdem Zwangskräfte im zu vermessenden Gelenk entstanden, die zu einer Verfälschung der Messergebnisse führten.

Die Führung über Lager bei Knösel beseitigte ferner die Fixierung einer ortsfesten Drehachse wie sie bei Stühmer vorlag, so dass die natürliche Bewegung der Tibia im Roll-Gleitvorgang mit wandernder Drehachse besser nachempfunden werden konnte.

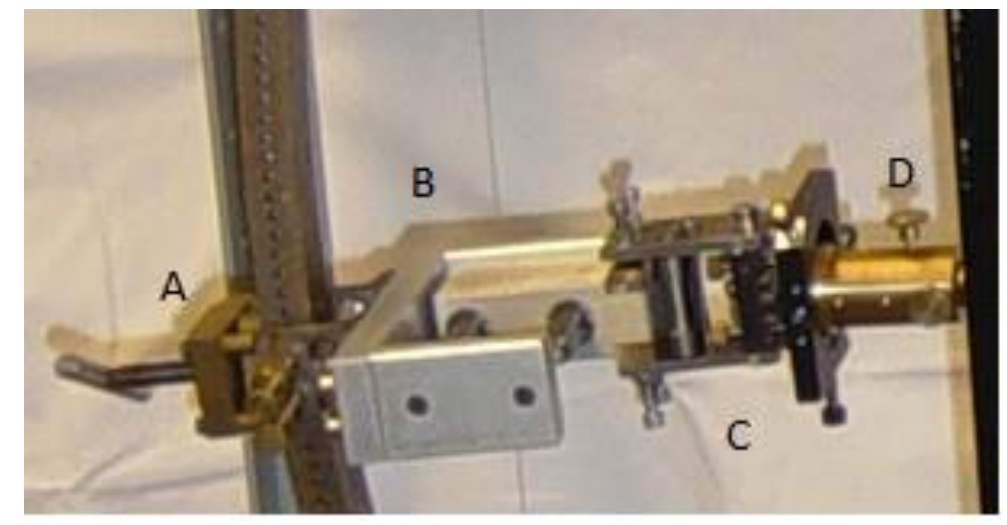

Abbildung 25: Arretierung nach Knösel (2003) an der Kreisbogenschiene.

A: Arretierung am Kreisbogen B: Linearlager zur Freischaltung der Freiheitsgrade (med.-lat. Translation, Rotation und Translation um tibiale Längsachse) C: Verbindungsteil zur Kraftmessdose D: Fixierschraube für die Kraftmessdose

\subsubsection{Anforderungen an die Konzeption}

Um die mechanische Stabilität von Knieendoprothesen messen zu können, mussten für die vorliegende Arbeit an der vorhandenen Apparatur folgende Voraussetzungen erfüllt sein:

- Physiologischer Bewegungsablauf: Die Messapparatur sollte die Voraussetzung liefern, die Knieendoprothesen mit ihrer Tibia- beziehungsweise Femurkomponente so zu befestigen, dass der dem natürlichen Knie physiologisch mögliche Flexions- und Extensionswinkelbereich weitgehend eingestellt werden konnte. Für die Untersuchung sollte eine geringe Auslenkung $\left(<1^{\circ}\right)$ aus der Gleichgewichtslage möglich sein. Dabei musste während der Auslenkung der Tibia gewährleistet sein, dass keine Zwangskräfte durch die Arretierung selbst oder das Eigengewicht auf das Gelenk ausgeübt werden. Ebenso musste die ausgelenkte Position für die Dauer des Messvorganges ohne Störungen und Zwangskräfte gehalten werden.

- Kraftübertragung: Um die physiologischen Kräfte der Hauptmuskeln, die das Kniegelenk beeinflussen, zur Auslenkung anwenden zu können, musste eine spe- 
zielle Anordnung geschaffen werden. Dazu sollte eine Vorrichtung zur Kraftuibertragung entwickelt werden, welche an den physiologischen Ansatzpunkten der Tibiakomponente befestigt werden kann, damit die Kraftwirkungslinie in Abhängigkeit vom eingestellten Flexionswinkel variabel und nach dem Modell der humanen Muskulatur verwirklicht werden konnte.

Die obigen Anforderungen an die Konzeption müssen erfüllt sein unter der Berücksichtigung, dass das Sensorsystem weiterhin in der Lage sein muss, die möglichen Bewegungen des Tibiofemoralgelenks im dreidimensionalen Raum aufnehmen zu können unter Berücksichtigung der entsprechenden Kalibrierung.

Im Folgenden werden die Änderungen beschrieben, die für die vorliegende Untersuchung notwendig waren.

\subsubsection{Modifikation des Messaufbaus}

\subsubsection{Die Messanordnung}

Für die Untersuchungen der vorliegenden Arbeit wurden einige Modifikationen vorgenommen, welche in Abbildung 26 zu sehen sind.

Am äußeren Gestell ist eine Kreisbogenschiene befestigt, die die Hauptbewegungsebene des Kniegelenks repräsentiert, in der Extensions- und Flexionsbewegungen ausgeführt werden. Über das äußere Gestell wurde das Femur im entsprechenden Flexionswinkel in dieser Ebene positioniert. Als Femur fungierte ein Aluminiumprofil, an dessen kaudalem Ende jeweils die femoralen Komponenten des AEQUOS und Mebio Scan-Knie mittels Palavit L-Kunststoff befestigt wurden. Die tibialen Komponenten der Endoprothesensysteme wurden ebenfalls mit Palavit L-Kunststoff am kranialen Ende eines Aluminiumprofils befestigt. An den Aluminiumprofilen befinden sich Haken mit Hilfe derer die Ansätze der Muskulatur simuliert wurden (Vergleiche Abbildung 31).

Das Femur blieb somit Teil des raumfesten Systems. Die Kreisbogenschiene, die in den vorherigen Arbeiten (Knösel 2003, Stühmer 2002, Mackeben 1997) als Führungsschiene für die Tibia diente, war auch in der vorliegenden Arbeit fest am Gestell fixiert. 


\subsubsection{Befestigung der Tibia}

Knösel verwendete Lager zur Befestigung der Tibia an der Kreisbogenschiene (Vergleiche Abbildung 25). Die Lager erhöhten die Masse der in Abbildung 24 dargestellten Arretierung um drei Kilogramm. Dies hatte deutliche Auswirkungen auf die Messung wie Vorversuche zeigten, so kam es unter anderem zu ungewollten Kontaktpunktverschiebungen der artikulierenden Gelenkflächen. Auch wurden mit diesem Lager nicht alle kleinen Freiheitsgrade freigeschaltet, so dass Zwangskräfte auftraten. So schreibt Knösel (2003, Seite 57f.):

„,Die Unterschätzung deren Bedeutung (Anmerkung der Autorin: vier Freiheitsgrade des Tibiofemoralgelenkes) führte zu einer Meßkonstruktion, die eine medial-laterale Translation der Kontaktpunkte sowie die longitudinale Rotation der Tibia einerseits unterdrückt, andererseits jedoch die durch das Zwingen des starren Körpers Tibia in die durch die Führungsschiene aufgespannte Funktionsebene entstehende und - entscheidender noch: auf die Gelenkposition in der Lateralebene zurückwirkende Kräfte und Drehmomente mit den Meßwerten aus der Extensions-Flexions-Ebene vermengt und somit das Meßergebnis wenn nicht verfälscht, so doch zumindest nicht eindeutig erscheinen läßt.“

Man kann vermuten, dass auch die Reibung - vor allem die Haftreibung - der Lager Einfluss auf die Messung hatte. Dieses Problem wurde behoben, indem auf die Befestigung der Tibia nach Knösel in diesem Versuchsaufbau verzichtet wurde. Die Tibia befand sich stattdessen freischwebend auf der Kreisbogenführungsschiene, lediglich durch die angreifenden Muskelkräfte kraftschlüssig positioniert (Siehe Abbildung 28). Durch diesen Umbau wurden auftretende Zwangskräfte während der Messung grundsätzlich ausgeschlossen. Eine umständliche und fehleranfällige Arretierung, bedingt durch das Eigengewicht der Lager, mittels der Fixierschrauben (Abbildung 25) wurde verhindert. Schon Knösel zeigte, dass das Festziehen dieser Arretierung zu einer Kontaktpunktveränderung im Tibiofemoralgelenk führen kann (Knösel 2003). Die Kontaktpunktänderung verändert den Gleichgewichtszustand des Tibiofemoralgelenks, wodurch die Messergebnisse verfälscht werden.

Daher kam die Idee, die „Fixierung“ der Tibia alleinig über den Kraftschluss zu realisieren, welcher durch Einstellung der „Muskelkräfte“ zu einem Gleichgewicht führt, so dass auf die ,alte Arretierungsapparatur““ (Abbildung 24) verzichtet werden konnte. 
Durch die Vereinfachung der Konstruktion ist es möglich, sämtliche Freiheitsgrade des Gelenks freizugeben. Eine Zwangsführung kann so sicher ausgeschlossen werden, so dass keine unphysiologischen Kräfte im Gelenk erzeugt werden, die wieder Einfluss auf den Gleichgewichtszustand hätten.

Diese Vorgehensweise hat den Vorteil, dass auch ohne Daten aus der Kraftmessdose sichergestellt ist, dass ein Gleichgewicht vorliegt, da sich die Tibia in Position befindet, weshalb ein Gleichgewicht vorliegen muss, sofern keine weitere Arretierung der Tibia vorliegt. Zudem ergibt sich keine Beeinflussung durch etwaige hohe Lager- oder Rollmomente.

Ein weiterer Vorteil in dieser Art der Erzeugung des Kraftschlusses besteht darin, dass auf Hilfsmittel zur Verbindung zwischen Kreisbogenschiene, Tibia und Kraftmessdose nahezu verzichtet werden kann (Abbildung 27). Einzig ein Magnet als Anschlag wurde verwendet (Abbildung 34). Dies führte zu einer deutlichen Gewichtsreduktion des Kraftmesssystems. Ferner konnte die Arretierung vom beweglichen Teil der Messapparatur in den unbeweglichen verschoben werden. Damit wurde ausgeschlossen, dass bei der Lageveränderung der Tibia Zwangskräfte und Erschütterungen auf das Kniegelenk ausgeübt werden.

Zur Messung wurden die zu untersuchenden Endoprothesen jeweils an den VierkantAluminiumprofilen befestigt, welche Femur- und Tibia-Schaft repräsentieren. In ihrer Ausrichtung wurde auf die jeweiligen OP-Anleitungen Rücksicht genommen (http://www.aap.de/de/Produkte/Endoprothetik/Knieendprothetik/Mebio_Scan/index. html; Zugriff am 04.02.2005).

Die Verbindung zwischen Tibia und Femur erfolgte kraftschlüssig allein durch die Kräfte der nachempfundenen Muskelzüge. Zu diesem Zweck des Kraftschlusses wurden analog den anatomischen Ansatzpunkten der Flexoren und Extensoren an der Tibia über Ösenschrauben geflochtene Kevlarseile (Firma Profil, Handelsname: XR, Durchmesser 0,12 $\mathrm{mm}$ ) fixiert.

\subsubsection{Gewichte zur Erzeugung der „Muskelkräfte“}

Verwendeten Mackeben, Stühmer und Knösel Wasser zur Erzeugung der „Muskelkräfte“, so wurden die „Muskelkräfte“ in der vorliegenden Arbeit mittels Eisengewichten simuliert. Durch Eisengewichte wurden Gewichtskräfte erzeugt, die 
mittels Karabinerhaken auf die Kevlarseile übertragen wurden. Über Umlenkrollen wurden die Kevlarseile zu den simulierten Ansatzstellen der Muskulatur geführt.

Aufgrund der höheren Dichte von Eisen konnte platzsparend mit geringem Materialaufwand gearbeitet werden. Zudem erhöhte die Wahl von Eisengewichten die Sicherheit der Messung, da Wasser in Kombination mit einer elektrischen Anordnung zu schwerwiegenden Zerstörungen führen kann. Eisengewichte erwiesen sich als deutlich praktikabler.

Ebenso waren höhere Gewichte notwendig, um den Kraftschluss des Gelenks zu erzeugen und ferner kamen die simulierten „Muskelkräfte“ den physiologischen näher.

Die Eisengewichte konnten entweder in Behältern (gelbe Eimer in Abbildung 27) oder direkt an den Seilen befestigt werden (Eisenscheiben in Abbildung 27). 


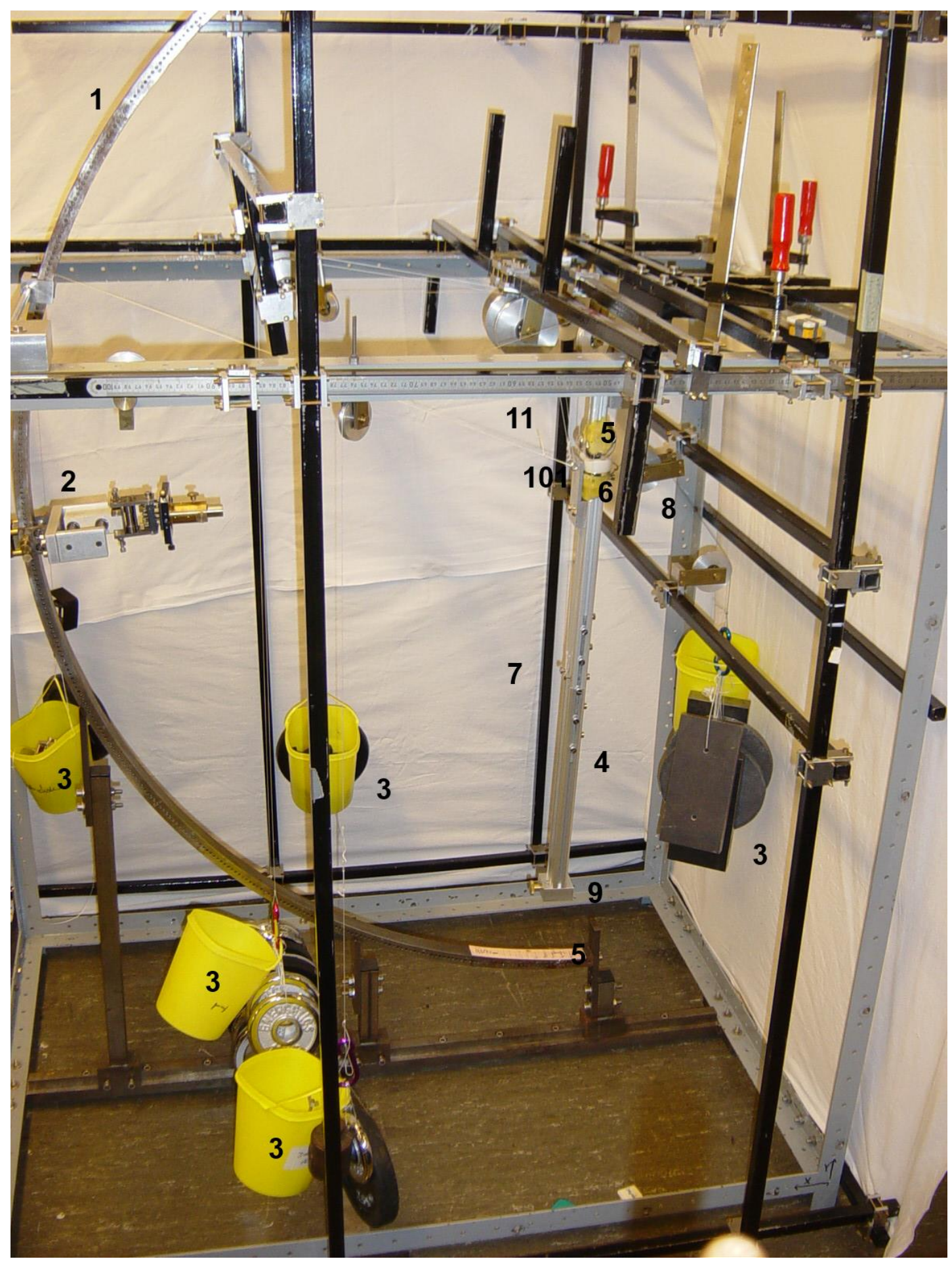

Abbildung 26: Messanordnung mit freischwebender Tibia im Gleichgewicht bei $5^{\circ}$ Kraftschluss, ohne Befestigung am Kreisbogen.

$1=$ Kreisbogenschiene $\quad 2=$ Arretierung nach Knösel/Stühmer $3=$ Eisengewichte $4=$ Tibia 5 = Femurkomponente des AEQUOS-Knie 6 = Tibiakomponente des AEQUOS-Knie mit Polyethyleninlay $7=$ Musculus gastrocnemius med und lat $8=$ Musculus quadriceps femoris mit Umlenkrolle 9 = Befestigung für Kraftmessdose (siehe Abbildung 30) $10=$ Musculus. popliteus $11=$ med. und lat. Hamstring. 


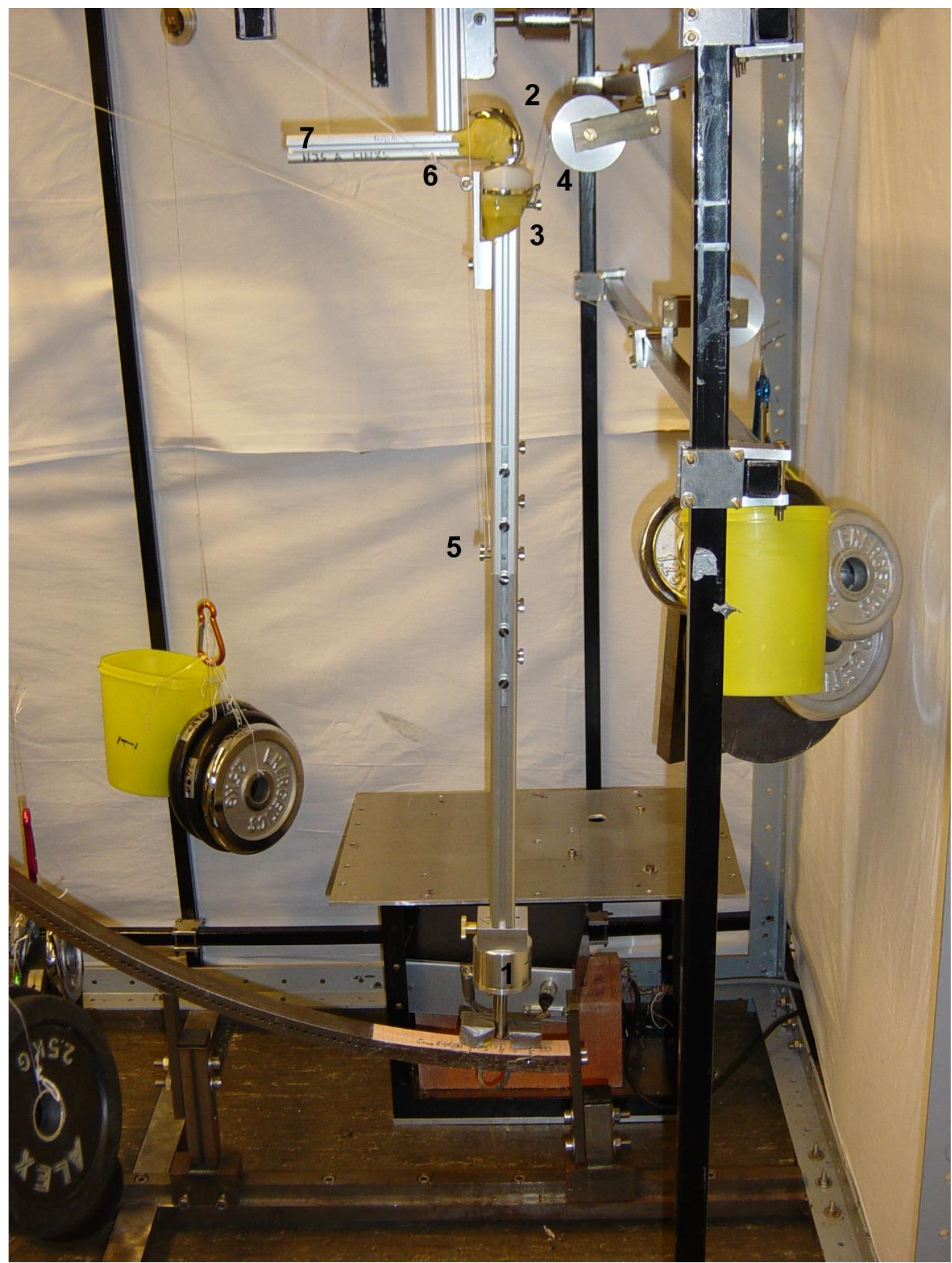

Abbildung 27: Messaufbau bei $90^{\circ}$ mit AEQUOS-Knie.

1=Kraftmessdose 2=Femurkomponente AEQUOS-Knie 3=Tibiakomponente AEQUOS-Knie 4=Musculus quadriceps femoris 5=med. und lat. Musculus. gastrocnemius 6=Musculus popliteus und med. und lat. Hamstring $7=$ Tibia Schaft

\subsection{Auslenkungsschritte}

Die Auslenkungsschritte der Tibia aus der jeweiligen Gleichgewichtsposition wurden von Knösel in $1^{\circ}$-Schritten gewählt und wurden in der vorliegenden Arbeit auf $0,33^{\circ}$ - 
Schritte reduziert, da eine Messung der Qualität und Quantität des eingestellten Gleichgewichts am sinnvollsten in den initialen Auslenkungsmomenten ist, das heißt die Auflösung wurde erhöht. Knösel arretierte am Kreisbogen, was technisch bedingt minimale Schritte von $1^{\circ}$ ermöglichte. Durch die Verwendung der Magnete konnten kleinere Schritte von $0,33^{\circ}$ gewählt werden.

\subsection{Kraftmessdose}

Der Prototyp der in der vorliegenden Arbeit verwendeten Kraftmessdose wurde von Planert et al. 1992 entworfen und von Mackeben (1997) und zuletzt Gerstenkamp (2016) modifiziert.

Hier soll zunächst das Prinzip der Kraftmessdose erläutert werden.

Der Kraftaufnehmer der Kraftmessdose besteht aus Blattfedern und aufgeklebten Dehnungsmessstreifen (DMS), welche die übertragenen Kräfte durch Änderung des elektrischen Widerstands in ein Spannungssignal umwandeln. Diese Verformung kann einem angreifenden Kraftsystem in allen Ebenen des Raumes zugeordnet werden, sodass die Kraftmessdose in der Lage ist drei Kräfte und drei Drehmomente im Raum aufzunehmen.

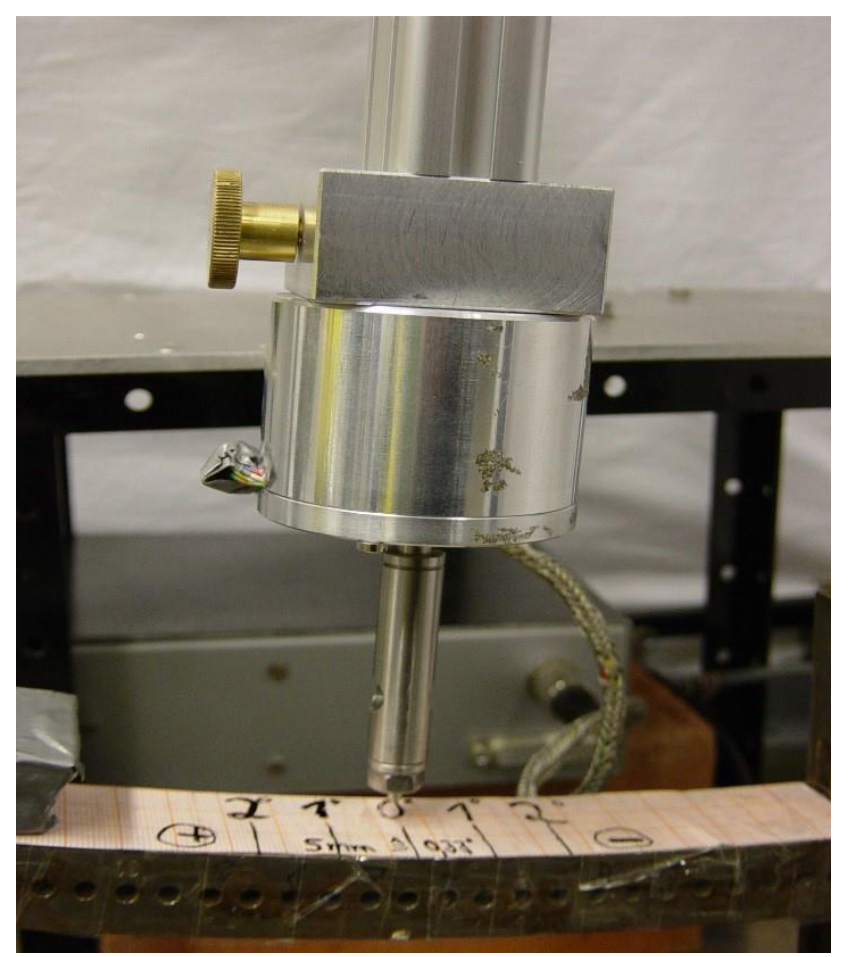

Abbildung 28: Messanordnung der Kraftmessdose. 
Von den DMS aus werden die elektrischen Signale in einen Verstärker geleitet, der dann die Signale in acht Kanälen an eine Schalteinheit übermittelt, von der aus über ein Potentiometer die jeweiligen Signale der acht Kanäle für die jeweilige Stellung der Tibia abgelesen werden.

Diese Werte wurden per Hand notiert und in einem statistischen Programm im Computer ausgewertet.

Die Kalibrierung erfolgte gemäß den von Gerstenkamp (2016) erarbeiteten Modifikationen. Die Auswertung der Messdaten wurde wie bei Mackeben (1997) über die Auftragung der ermittelten Kräfte beziehungsweise Drehmomente zur Auslenkung in Grad vorgenommen.

\subsection{Versuchsaufbau und -durchführung}

Die zu vergleichenden Knieendoprothesen wurden bei Flexionswinkeln von $5^{\circ}$ und $90^{\circ}$ mit unterschiedlichen isotonischen Kraftsystemen belastet, um die Veränderung des Gleichgewichtzustandes bei differierenden Kombinationen der „Muskelkräfte“ zu untersuchen. Dafür wurden, ausgehend von einem eingestellten Gleichgewichtszustand, möglichst kleine Auslenkungen in 0,33 ${ }^{\circ}$-Schritten in Flexions- und Extensionsrichtung manuell vorgenommen und dabei die auftretenden Kräfte und Drehmomente mittels der Kraftmessdose registriert.

Dabei ist das Femur fest verankert und die Tibia frei beweglich. Für die Flexionswinkel $5^{\circ}$ und $90^{\circ}$ wurden mittels der imitierten Muskelkräfte Gleichgewichtslagen aufgesucht, indem die einzelnen Muskelzüge nach und nach belastet wurden, bis sich visuell ein Gleichgewicht einstellte, in dem die Tibia kraftschlüssig am Femur im Gleichgewicht blieb, ohne dass sie von extern fixiert wurde. Dabei wurden die „kleinen" Freiheitsgrade, die beiden Rotationen um die Normalen in den beiden Kontakten, zugelassen, da es keine Zwangsführung gab. Insgesamt wurden sechs Muskelkräfte mit vorgegebenen Kraftwirkungslinien verwendet, die der physiologischen, anatomischen Realität entsprechen (Vergleiche Abbildung 32). Da mehr Kräfte als kinematische Freiheitsgrade vorhanden sind, können mit unterschiedlichen Muskelkombinationen verschiedene Gleichgewichte bei vorgegebenem Flexionszustand erzeugt werden. Diese Gleichgewichte unterscheiden sich im Ausmaß der Stabilität/Instabilität. Beim Messvorgang fand eine Auslenkung aus der eingestellten Gleichgewichtslage in 
vorgegebenen kleinen Flexions- und Extensionswinkeln statt, und das erzeugte Kraftsystem wurde aufgezeichnet.

\subsubsection{Fixierung des Untersuchungsmaterials}

$\mathrm{Zu}$ Beginn der Messreihen wurde zunächst die femorale Komponente der Endoprothese mit dem Aluminiumprofil, welches den Femurschaft simulierte, verbunden. Ebenso wurde die Tibiakomponente an das Aluminiumprofil angebracht, welches die Tibia darstellte. Zur Fixierung wurde ein Epoxidharzkleber und das kaltpolymerisierende Polymethylmethacrylat Palavit-L verwendet. Diese Schritte wurden jeweils für das AEQUOS-Knie (Abbildung 29) sowie für das Mebio Scan-Knie (Abbildung 30) durchgeführt.

Diese Anordnung blieb für die Dauer aller Messreihen konstant.

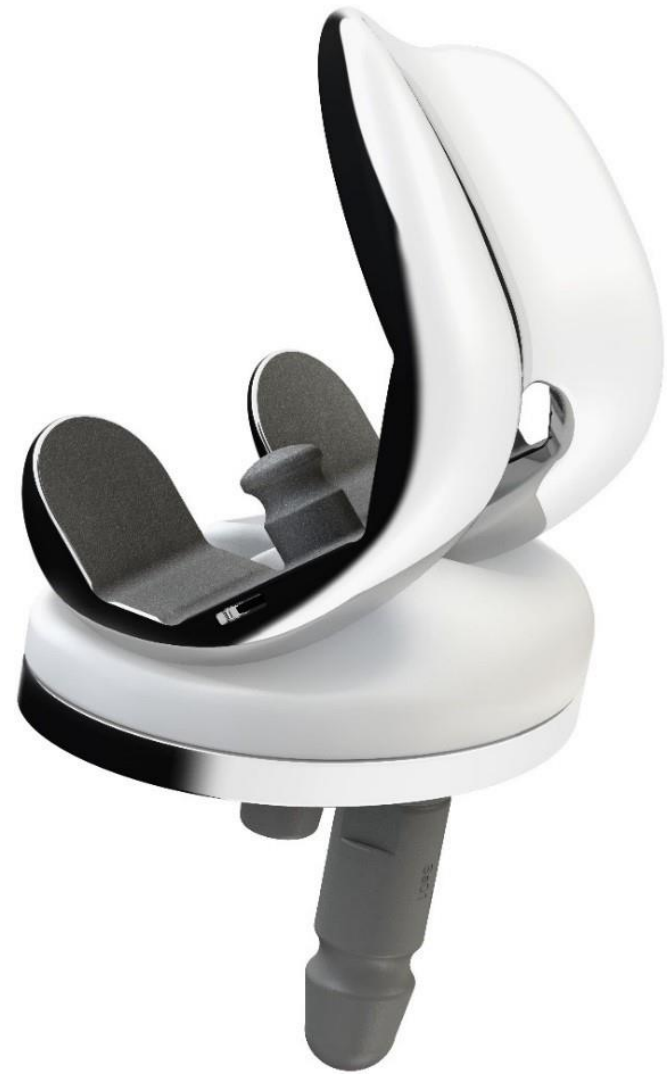

Abbildung 29: AEQUOS-Knie, Femurkomponente (oben), Patellakomponente (Mitte), Tibiakomponente (unten).

(http://www.neu.unibayreuth.de/de/Uni_Bayreuth/Fakultaeten/6_FAN/Metallische Werkstoffe neu unfertig/de/research/polymer/index.html; Zugriff am 09.04.2018). 


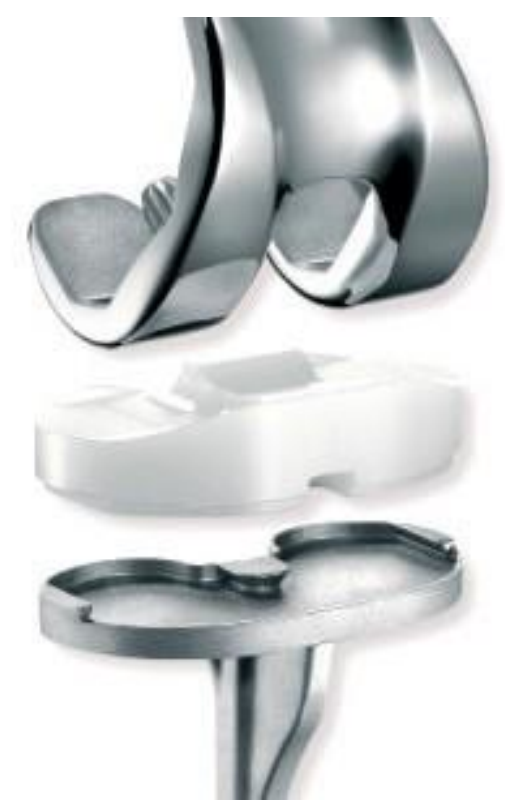

Abbildung 30: Mebio Scan-Knie.

(http://www.aap.de/de/Produkte/Endoprothetik/Knieendprothetik/Mebio_Scan/index.html; Zugriff am 04.02.2005).

Das Aluminiumvierkantprofil mit der fixierten Femurkomponente des jeweiligen Endoprothesensystems stellte das Femur dar und wurde fest am äußeren Rahmen der Messapparatur befestigt (Abbildung 27). 


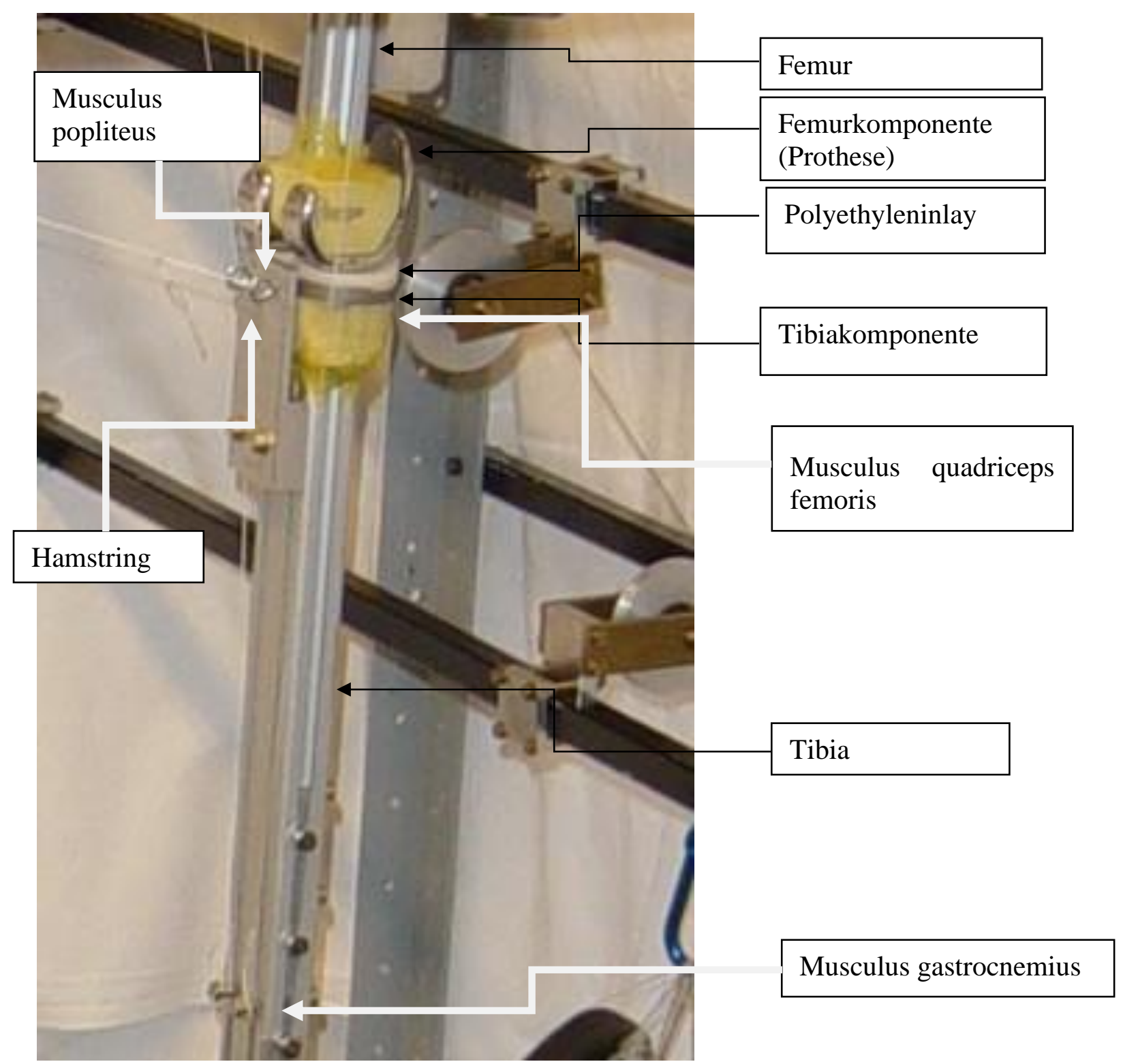

Abbildung 31: Befestigung der Endoprothese.

Die Abbildung zeigt die Tibia und Femurkomponente, welche mittels Kunststoff (gelb) an zwei Aluminiumprofilen, welche Tibia und Femur darstellen, befestigt sind. Die Ösenschrauben stellen die Ansätze der Muskelkräfte dar. An ihnen sind über weiße Seile die Muskelkräfte angebracht.

Der Kontaktpunktbereich wurde nun mit Kriechöl behandelt, um die Reibung zu minimieren und die natürliche Schmierung durch die Synovialflüssigkeit zu simulieren.

\subsubsection{Herstellung von Gleichgewichtslagen}

Über Kevlarseile und Umlenkrollen konnten die Muskelkräfte des Musculus Quadriceps femoris, der Hamstring, mit medialer und lateraler Komponente, des Musculus gastrocnemius mit medialer und lateraler Komponente, und die Kraft des Popliteus auf 
das Kniegelenk übertragen werden. Dabei wurden die Kraftwirkungslinien der entsprechenden Muskeln gemäß derer physiologischen Ansätze und Ursprünge angeordnet.

Um die Messung durchführen zu können, mussten die Muskelzüge über Umlenkrollen geleitet werden, damit die Gewichte genug Abstand zur Kraftmessdose bzw. der Tibia haben. Die Kevlarseile konnten aufgrund der Führungsnut auf den Umlenkrollen so positioniert werden, dass die physiologische Krafteinwirkung der jeweiligen Muskelzüge eingestellt werden konnte. Es wurden die Extensorengruppe (Musculus quadriceps), die Flexoren- oder Hamstringgruppe sowie der Musculus gastrocnemius und der Musculus popliteus nachempfunden. Auf diese Weise ist ein isotonisches Kraftsystem möglich.

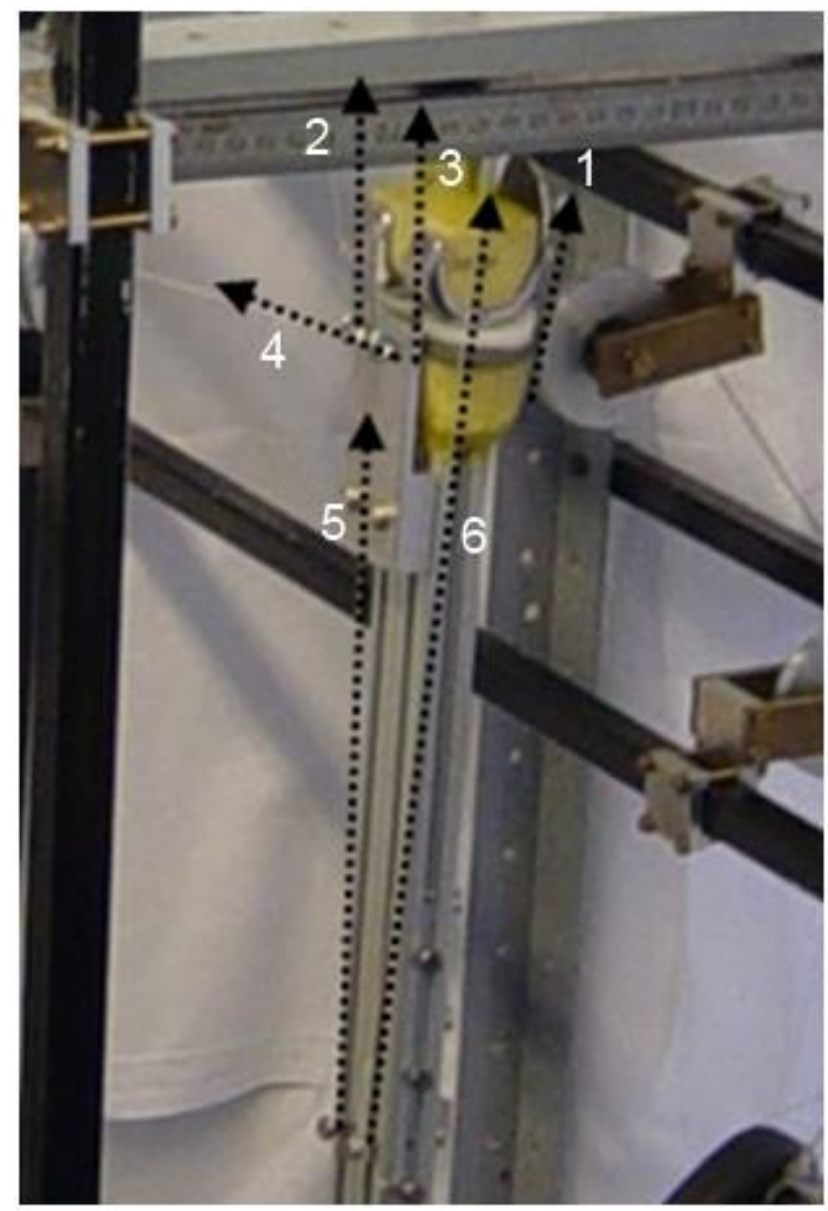

Abbildung 32: Kraftschluss im Gelenk in Gleichgewichtslage mit eingezeichneten Ansätzen und Zugrichtungen der Kevlarseile.

$1=$ Musculus quadriceps 2 = laterale Hamstring 3 = mediale Hamstring 4 = Musculus popliteus 5 = lateraler Musculus gastrocnemius $6=$ medialer Musculus gastrocnemius

Nachdem die Kevlarseile an den Ösen befestigt und über Umlenkrollen geführt worden waren, wurden mehrere Eisengewichte an die Karabinerhaken gehängt und 
kleine Eisengewichte befanden sich in gelben Eimern (Abbildung 27). Die Summe der Gewichtskräfte zur Simulation der „Muskelkräfte“ war für alle Messreihen gleich.

Die Zugkraft der Seile stellte den gewünschten Flexionswinkel ein und sicherte zugleich den Kraftschluss im Gelenk.

Hinsichtlich der Frage des Einflusses der Reibung - insbesondere der Haftreibung auf die Messung, wird an dieser Stelle auf die Arbeit von Mackeben verwiesen, der diese als vernachlässigbar klein bewertet (Mackeben 1997).

Die Tibia wurde per Hand mit dem Femur in Kontakt gebracht. Erst jetzt wurden über die Ansätze der Muskulatur gleichmäßig Gewichte auf die verschiedenen virtuellen Muskeln, das heißt auf die entsprechenden Seile, verteilt. Die Tibia wurde nun mit Hilfe der Kevlarseile über physiologische Kräfte in Kontakt zum Femur positioniert (Vergleiche Abbildung 32).

Die so ermittelte optimale Tibiaposition wurde in dem voreingestellten Flexionswinkel noch so lange manuell unterstützt, bis die Gewichtskräfte appliziert wurden, die eine Gleichgewichtsposition herstellen konnten. Die Muskelkräfte wurden so lange manuell variiert, bis eine Gleichgewichtslage eingestellt war. Dazu wurde bei der Applikation der Gewichtskräfte die Tibia zunächst manuell fixiert und nach jeder Testeinstellung sequentiell losgelassen, um $\mathrm{zu}$ kontrollieren, ob eine Gleichgewichtsposition erreicht war.

Ein Gleichgewicht wurde darüber erkannt, dass die Tibia stationär am Femur unter Kraftschluss positioniert war, ohne dass eine manuelle Manipulation weiterhin notwendig war.

Sowohl bei $5^{\circ}$ als auch bei $90^{\circ}$ Flexion wurden unterschiedliche Kraftvarianten durch Änderung der Verteilung der Muskelkräfte auf das System aufgebracht, um die Veränderung der Qualität der Gleichgewichtszustände nachweisen zu können. Es wurde in der Reihenfolge AEQUOS-Knie - Mebio Scan-Knie gearbeitet. Die Kraftvariante, die bei dem AEQUOS-Knie zu einer Gleichgewichtslage geführt hatte, wurde als Anhaltspunkt für das Kräftesystem der Mebio Scan-Endoprothese genommen und stellte den Ausgangspunkt für die Gleichgewichtseinstellung dort dar. Nun wurde am kaudalen Ende der Tibia über der Kreisbogenschiene die Kraftmessdose mit dem Zentrierdorn mit Hilfe einer Gewindeschraube befestigt und die Nullpunktmessung durchgeführt. Die Kraftmessdose wurde so justiert, dass die z- 
Achse orthogonal durch das Gelenk verläuft, die Hauptfunktionsebene des Tibiofemoralgelenks wird durch die y-z-Ebene aufgespannt (Abbildung 33).

Der Nullpunkt des Koordinatensystems liegt in der Kraftmessdose. Der Abstand des Nullpunktes zu den Kontaktpunkten beträgt 80,2 cm.

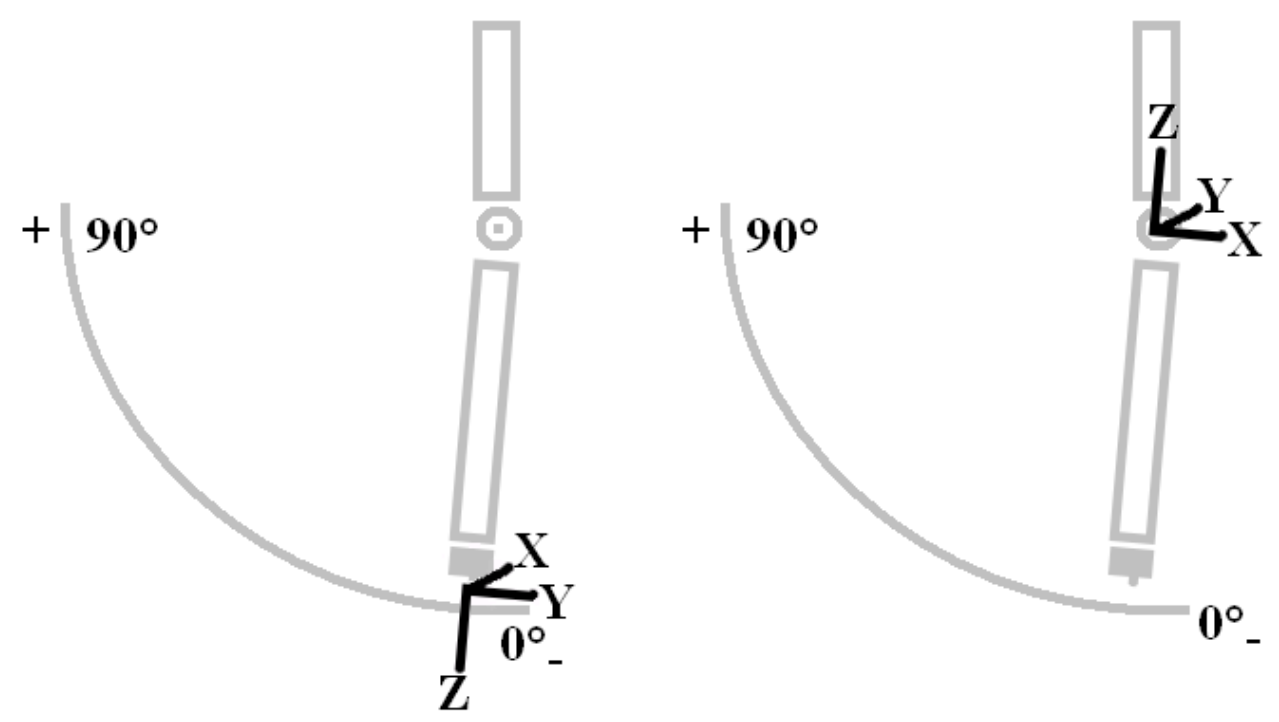

Abbildung 33: Koordinatensystem in der Kraftmessdose und im Kniegelenk nach Gerstenkamp (2016). Der Abdruck erfolgt mit freundlicher Genehmigung von Dr. G. Gerstenkamp.

Die in der Kraftmessdose registrierten Kräfte und Drehmomente sind nicht identisch mit den im Kniegelenk bzw. den Endoprothesen wirkenden Kräften und Drehmomenten. Das dort wirkende Kraftsystem muss unter Beachtung des Abstandes von $80,2 \mathrm{~cm}$ und der Orientierung im Tibiofemoralgelenk rechnerisch ermittelt werden.

\subsubsection{Messung}

Bei jeder Messung (1-9; Siehe Kapitel 4) wurde zunächst eine Nullpunktmessung durchgeführt, die als Referenz zu den Messungen während der Auslenkung bei gleichbleibendem isotonischem Kraftsystem diente. Das heißt, die Ausgangssignale der Kraftmessdose wurden registriert, wenn die jeweilige Knieendoprothese kraftschlüssig durch die „Muskelkräfte“ positioniert war. Dabei war der Ausgangswinkel der Messungen 1-5 ein Flexionswinkel von $5^{\circ}$ und bei den 
Messungen 6-9 ein Flexionswinkel von $90^{\circ}$. Durch Variationen der Eisengewichte an den simulierten „Muskelkräften“ konnten bei jeder Messung unterschiedliche Kraftwirkungslinien erzeugt werden, die die jeweilige Gleichgewichtsposition der Knieendoprothesen erzeugten.

Nach der Aufzeichnung der Nullpunktmessung wurde, ausgehend von dem Beugungswinkel von $5^{\circ}$ oder $90^{\circ}$, eine Auslenkung per Hand an der Kraftmessdose durchgeführt. Diese manuelle Auslenkung fand in $0,33^{\circ}-$ Schritten statt $\left(+0,33^{\circ} /\right.$ $0,33^{\circ} ;+0,67^{\circ} /-0,67^{\circ} ;+1,0^{\circ} /-1,0^{\circ} ;+1,33^{\circ} /-1,33^{\circ} ;+1,67^{\circ} /-1,67^{\circ} ;+2,0^{\circ} /-$ $\left.2,0^{\circ}\right)$

Dabei wurde der Zentrierdorn ausgehend von der Stellung der Nullpunktmessung mit dem Finger um zunächst $0,33^{\circ}$ entlang der Kreisbogenschiene bewegt. Als Anschlag diente ein Magnet, der auch zur Arretierung diente. Jeder oben aufgeführte Auslenkungsschritt wurde manuell eingestellt und die auftretenden Kräfte wurden registriert. (Abbildung 34).

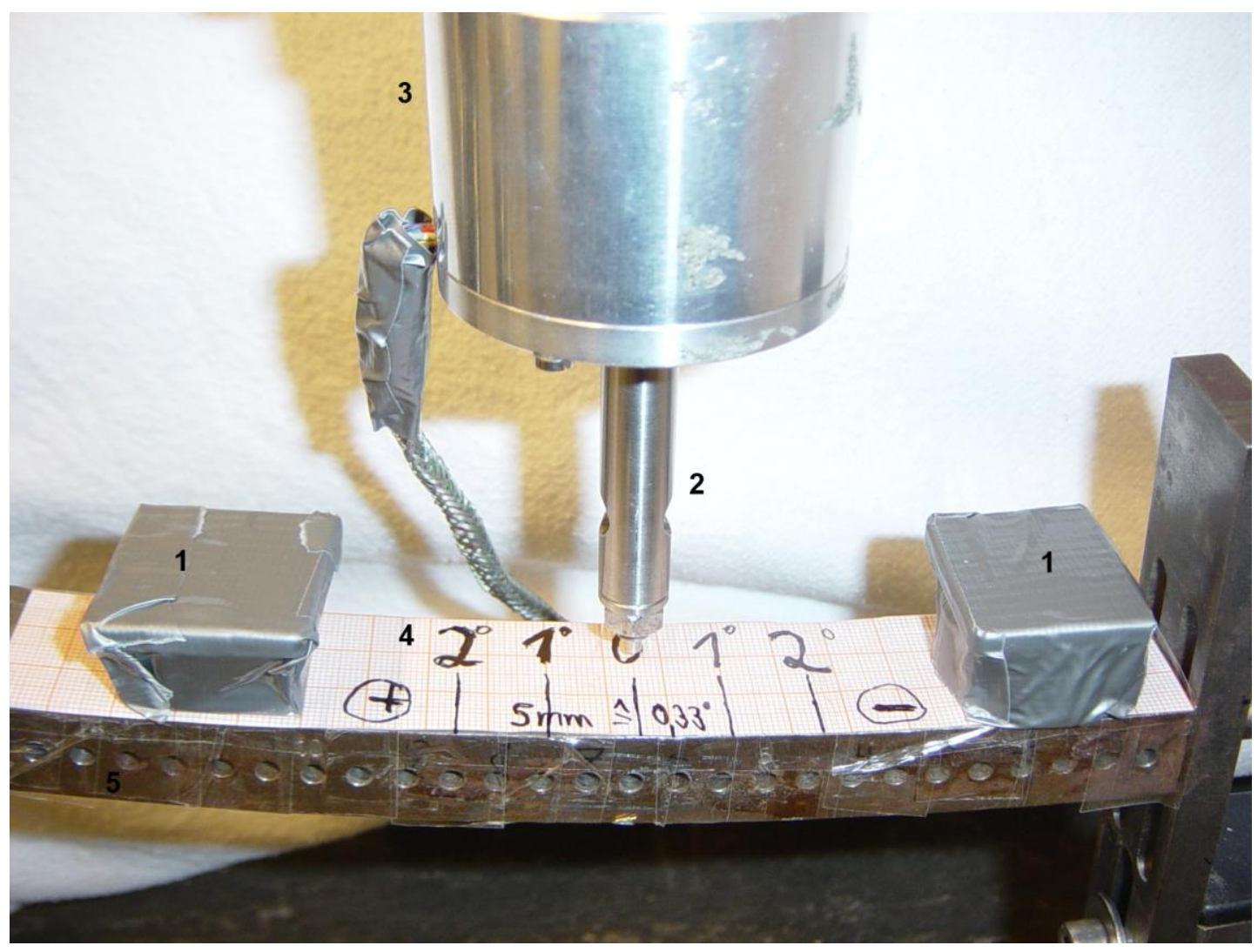

Abbildung 34: Detailaufnahme im Gleichgewichtszustand der Kreisbogenschiene mit Magneten zur Arretierung vor Auslenkung.

1 = Magnet, dient als Anschlag, 2 = Zentrierdorn ohne Kontakt zum Kreisbogen, $3=$ Kraftmessdose, $4=$ Millimeterpapier, $5=$ Kreisbogenschiene 
Nach dem Einstellen der gewählten Auslenkung (negativ/positiv) wurden die Messwerte aufgenommen, das heißt die Kräfte, die nach der Auslenkung herrschten. Die Tibia wurde erneut in die Gleichgewichtslage im Ausgangswinkel geführt und die Einzelmessung wurde viermal pro Auslenkung wiederholt.

Nach dem Abschluss einer Messung wurde in die $0^{\circ}$ Gleichgewichtslage zurückgekehrt.

Die Messungen 1-9 setzen sich demnach jeweils aus den Messwerten an den Auslenkungsschritten von:

$+0,33^{\circ} /-0,33^{\circ} ;+0,67^{\circ} /-0,67^{\circ} ;+1,0^{\circ} /-1,0^{\circ} ;+1,33^{\circ} /-1,33^{\circ} ;+1,67^{\circ} /-1,67^{\circ}$; $+2,0^{\circ} /-2,0^{\circ}$

zusammen.

Die vier Einzelwerte eines viermalig wiederholten Auslenkungsschrittes ergaben eine Messung. Die Messergebnisse wurden gemittelt und die Differenz zum Nullwert errechnet. 


\section{$4 \quad$ Ergebnisse}

\subsection{Interpretation der Ergebnisse}

\subsubsection{Physikalischer Hintergrund}

Durch ihren Aufbau ist die Kraftmessdose in der Lage, in allen sechs Dimensionen des Raumes zu messen, somit werden drei Kräfte und drei Drehmomente registriert. Diese Kräfte werden aber in der Kraftmessdose gemessen, welche nicht gleich den wirkenden Kräften im Kniegelenk beziehungsweise den Endoprothesen ist. Die dort wirkenden Kräfte müssen unter Beachtung des Abstandes zwischen Gelenkmittelpunkt und Kraftmessdose von 80,2 cm rechnerisch ermittelt werden.

Die Ergebnisse der Messungen werden in Kapitel 4.2 graphisch dargestellt. Zu jeder Messung wird eine Tabelle mit dem jeweiligen individuell applizierten Muskelkraftsystem dargestellt.

Die Graphen zeigen zwei Kurven (rot $\mathrm{F}(\mathrm{x})$ und grün $\mathrm{T}(\mathrm{y})$ ) und eine Tangente im Nullpunkt, hier Stabilitätstangente genannt. Die rote Kurve stellt die Qualität der Kräftestabilität dar und die grüne die Qualität der Drehmomentstabilität. Die Abszisse zeigt die Auslenkungsabfolge in $0,33^{\circ}$-Schritten, von der Ausgangslage ( $5^{\circ}$ oder $90^{\circ}$ ) als 0 Punkt bezeichnet bis $2^{\circ}$. Dabei hat die zunehmende Flexion ein positives Vorzeichen und die Extension ein negatives Vorzeichen.

Die linke $y$-Achse des Graphen stellt das gemessene Drehmoment in Newtonzentimetern dar und die rechte y-Achse die registrierte Kraft in Newton.

Dabei wird die Qualität des Gleichgewichts durch das Vorzeichen des entsprechenden Differentialquotienten der Auslenkung aus der Gleichgewichtslage bestimmt. Der Differentialquotient selbst zeigt die Quantität des Gleichgewichts.

Für die Interpretation der Kurven gilt: Ein stabiles Gleichgewicht ist durch eine positive Rückstellkraft und ein rücktreibendes Drehmoment gekennzeichnet. Das heißt, bei Vergrößerung der Flexion ist ein stabiles Gleichgewicht durch eine positive Rückstellkraft $+\mathrm{F}(\mathrm{x})$ und ein Drehmoment $-\mathrm{T}(\mathrm{y})$ gekennzeichnet. Bei einer Auslenkung in negativer $x$-Richtung gleichbedeutend mit einer Zunahme der Extension ist der stabile Zustand durch ein $+\mathrm{T}(\mathrm{y})$ und eine negative Kraft $-\mathrm{F}(\mathrm{x})$ in Richtung auf den Mittelpunkt gekennzeichnet.

Ein instabiles Gleichgewicht zeigt eine forttreibende Kraft und ein forttreibendes 
Drehmoment. Bei Auslenkung in eine größere Flexion bedeutet dies eine forttreibende Kraft $-\mathrm{F}(\mathrm{x})$ und das Drehmoment $+\mathrm{T}(\mathrm{y})$ und bei Auslenkung in Extension durch $-\mathrm{T}(\mathrm{y})$ und $+\mathrm{F}(\mathrm{x})$.

Die Graphen zeigen zudem die Stabilitätstangente durch den Nullpunkt: Für eine Quantifizierung des Stabilitätsgrades wurde die Steigung der Tangente im Nullpunkt (Ausgangslage) ermittelt. Diese stellt eine Ableitung ersten Grades im Nullpunkt dar und wird gemittelt aus den Grenzwerten $+0,33^{\circ}$ und $-0,33^{\circ}$.

Ein indifferenter Zustand wird durch eine Tangente mit der Steigung 0 dargestellt. Dabei erhöht sich die Labilität, je steiler F`(x) steigt und die Gerade von T`(y) fällt.

Das Ausmaß der Stabilität erhöht sich, je steiler die Gerade $\mathrm{T}^{\prime}(\mathrm{y})$ ist und je ausgeprägter die Gerade F(x) deszendiert.

An der Steigung der Tangente kann sowohl die Qualität des Gleichgewichts als auch deren Ausprägung abgelesen werden.

Im Anhang finden sich zwei Tabellen (Tabelle 10 und Tabelle 11) zur Übersicht der Kräfte und Stabilitätsgrade. In Tabelle 11 wird das Verhältnis der Muskelkräfte zueinander dargestellt.

\subsubsection{Beobachtungen}

Während der Versuchsdurchführung erwies es sich als schwierig, beim Mebio ScanKnie im Gegensatz zum AEQUOS-Knie Gleichgewichtspositionen zu finden und einzustellen.

Es war schon während des Versuchsaufbaus bei der Positionierung der Tibia zum Femur auffällig, dass die Kontaktbildung zwischen Femurkomponente und Tibiakomponente beim AEQUOS-Knie sofort zu einer kraftschlüssigen Position führte, die frei ohne die Aufbringung von Zwangskräften stabil blieb. Demgegenüber war es mühsam, für die tibiale Komponente des Mebio Scan-Knies die Gleichgewichtspositionierung herzustellen. Diese Kontaktpunkte-Position erschien auch beliebig, das heißt sie war nicht konstant zu halten und konnte sich während eines Versuchsdurchlaufs ändern. Dabei konnte beobachtet werden, dass die Kontaktbeziehung zwischen femoraler und tibialer Komponente des Mebio ScanKnies nicht klar definiert war, es kam zu einem ständigen Hin- und Hergleiten der Tibia auf dem Femur und damit einer Kontaktpunktverschiebung. 
Im Zuge dieser Vorbereitung konnten schon visuell unterschiedliche Gleichgewichtszustände beim AEQUOS-Knie festgestellt werden. So stellte sich ein stabiler Zustand ein, bei dem die Tibia nach geringer Auslenkung in die Ausgangslage zurückwanderte. Auch labile Gleichgewichtszustände waren möglich, die dazu führten, dass eine Auslenkung eine Reaktion des Systems initiierte, die die Auslenkung vergrößern wollte.

Hierdurch konnte die 1990 von Nägerl (Nägerl 1990, Nägerl et al. 1993) postulierte Funktionsweise des Kniegelenks nachgewiesen werden, da das AEQUOS-Knie dem natürlichen Kniegelenk entspricht.

Diese visuellen Beobachtungen konnten durch die messtechnische Auswertung validiert werden. Beim Mebio Scan-Knie war zu beobachten, dass regelmäßig eine nicht gewollte Hyperextension des Kniegelenks auftrat. Das heißt, dass das Gelenk nach vorne wegrutschte. Es kam zu einem unkontrollierten Verschieben der Kontaktpunkte nach ventral bis hin zu einem Herausgleiten der Tibiakomponente aus dem Femurlager.

Dies kann damit zusammenhängen, dass das Mebio Scan-Knie eine dimere Kette darstellt, da eine mediale und laterale Symmetrie der Komponenten vorliegt. Es existiert eine Femurachse und eine Tibiaachse, die einen konstanten Abstand halten. Ein Gleichgewicht ist gegeben, wenn die Kraftwirkungslinie die kompressive Gelenkkraft beider Achsen schneidet.

Beim AEQUOS-Knie muss die kompressive Kraft hingegen lediglich durch die momentane Drehachse verlaufen, um ein Gleichgewicht zu erzeugen, was leichter zu bewerkstelligen ist.

Das Mebio Scan-Knie reagierte sehr viel empfindlicher auf die angreifenden Muskelkräfte als das AEQUOS-Knie. Das heißt, bei der Einstellung einer Gleichgewichtslage „rutschten“ die Kontakte bei der Applikation der Gewichte. Bei dem AEQUOS-Knie konnte eine Selbststabilisierung beobachtet werden, die zu definierten Kontaktbeziehungen führte.

Auch war beim AEQUOS-Knie ein deutliches Abrollen der artikulierenden Endoprothesenoberflächen zu beobachten, während es beim Mebio Scan-Knie zu einem Gleiten/Rutschen kam.

Die Ergebnisse für die Flexionswinkel von $5^{\circ}$ und $90^{\circ}$, jeweils eingestellt am 
AEQUOS-Knie und am Mebio Scan-Knie in insgesamt neun Messungen werden im Folgenden dargestellt.

\subsection{AEQUOS-Knie}

\subsubsection{Flexionswinkel $5^{\circ}$}

\subsubsection{Messung 1}

Bei der Messung des AEQUOS-Knie im Flexionswinkel von $5^{\circ}$ zeigten die Auslenkungen zwischen $+2^{\circ}$ und $-2^{\circ}$ ein ausgeprägtes stabiles Gleichgewicht an, mit etwas größeren Rückstellkräften bei der Auslenkung in positiver x-Richtung. Es ergab bei $+2^{\circ}$ eine maximale Kraft $\mathrm{F}(\mathrm{x})$ von $0,84 \mathrm{~N}$ sowie ein Drehmoment von $-71,6 \mathrm{Ncm}$, bei $-2^{\circ}$ entsprechend $-0,68 \mathrm{~N}$ bzw. 58,1 Ncm. Der Stabilitätsgrad betrug -0,69 N/Grad für die Kräfte und 56,89 Ncm/Grad für das Drehmoment (Abbildung 35 und Tabelle $10)$.

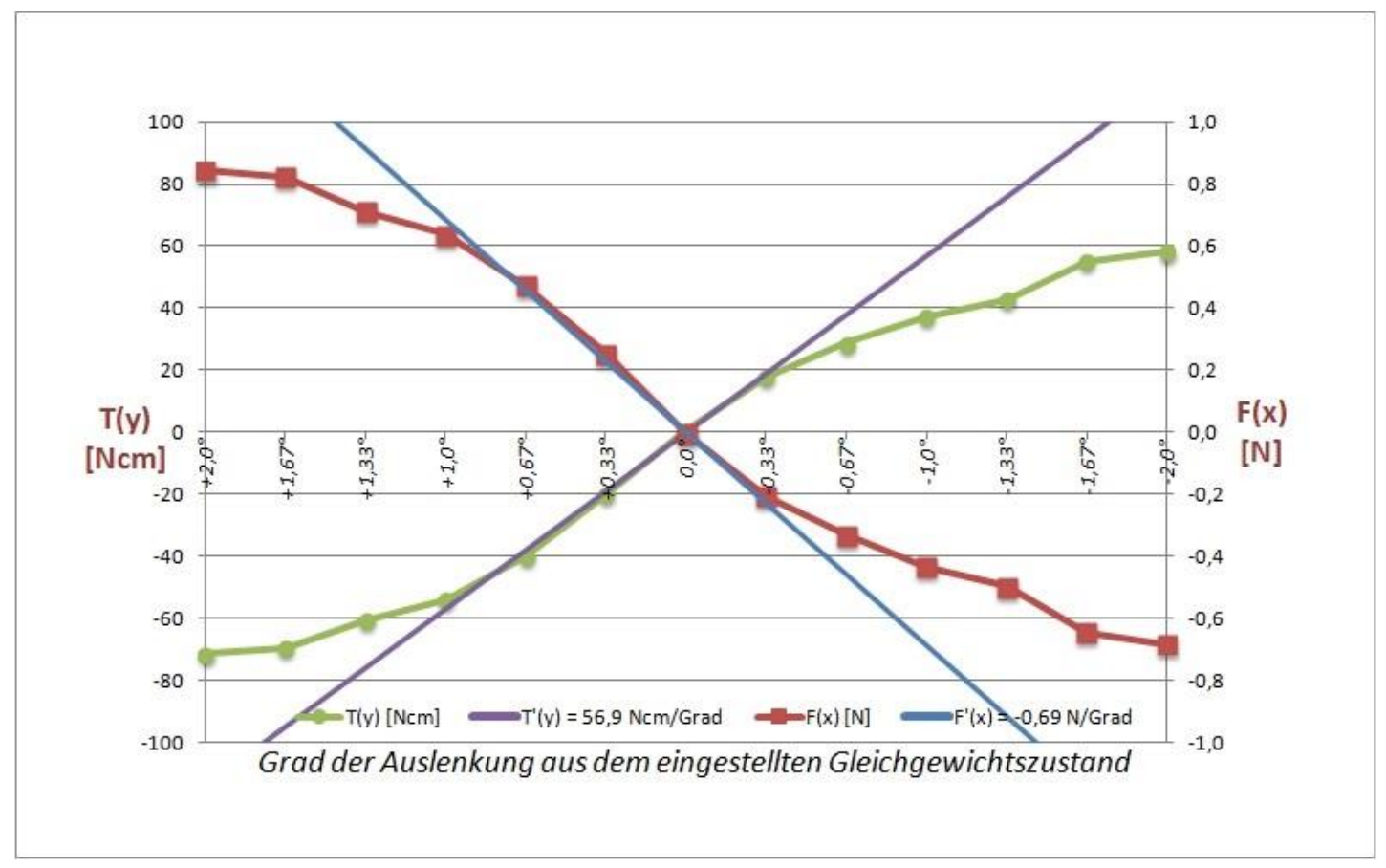

Abbildung 35: Resultierende Kraft und Drehmoment für die AEQUOS-Knie bei Auslenkungen im Flexionswinkel $5^{\circ}$, Messung 1.

In der hier eingestellten Kraftvariante wurde mit einem Verhältnis von Hamstring zu Musculus gastrocnemius von 2,6 gearbeitet (Tabelle 1 und Tabelle 11).

Laterale und mediale Kräfte wurden annähernd gleich eingestellt. 


\begin{tabular}{|l|c|c|c|c|c|c|}
\hline & Quadriceps & \multicolumn{2}{|c|}{ Hamstring } & \multicolumn{2}{c|}{ Gastrocnemius } & Popliteus \\
\hline & & Medial & Lateral & Medial & Lateral & \\
\hline F (N) & 203,01 & 129,8 & 130,7 & 45,9 & 53,5 & 57,1 \\
\hline
\end{tabular}

Tabelle 1: Appliziertes Kräftesystem bei der Messung 1.

\subsubsection{Messung 2}

In dieser Kraftvariante stellte sich im AEQUOS-Knie sowohl im Extensions- als auch im Flexionsbereich ebenfalls ein stabiles Gleichgewicht mit einem maximal resultierenden Drehmoment von $51,5 \mathrm{Ncm}$ beziehungsweise $-36,3 \mathrm{Ncm}$ ein, die rücktreibenden Kräfte waren kleiner als bei der Kraftvariante 1. Die Auslenkungen führten also zu einer geringeren Stabilität als bei Messung 1.

Bei einer Auslenkung von $+2^{\circ}$ in Flexionsrichtung resultierte ein $\mathrm{F}(\mathrm{x})$ von $0,45 \mathrm{~N}$, und bei $-2^{\circ}$ von $-0,65$ N. Der Stabilitätsgrad betrug $-0,55$ N/Grad für die Kräfte und 45,42 Ncm/Grad für das Drehmoment (Abbildung 36 und Tabelle 10).

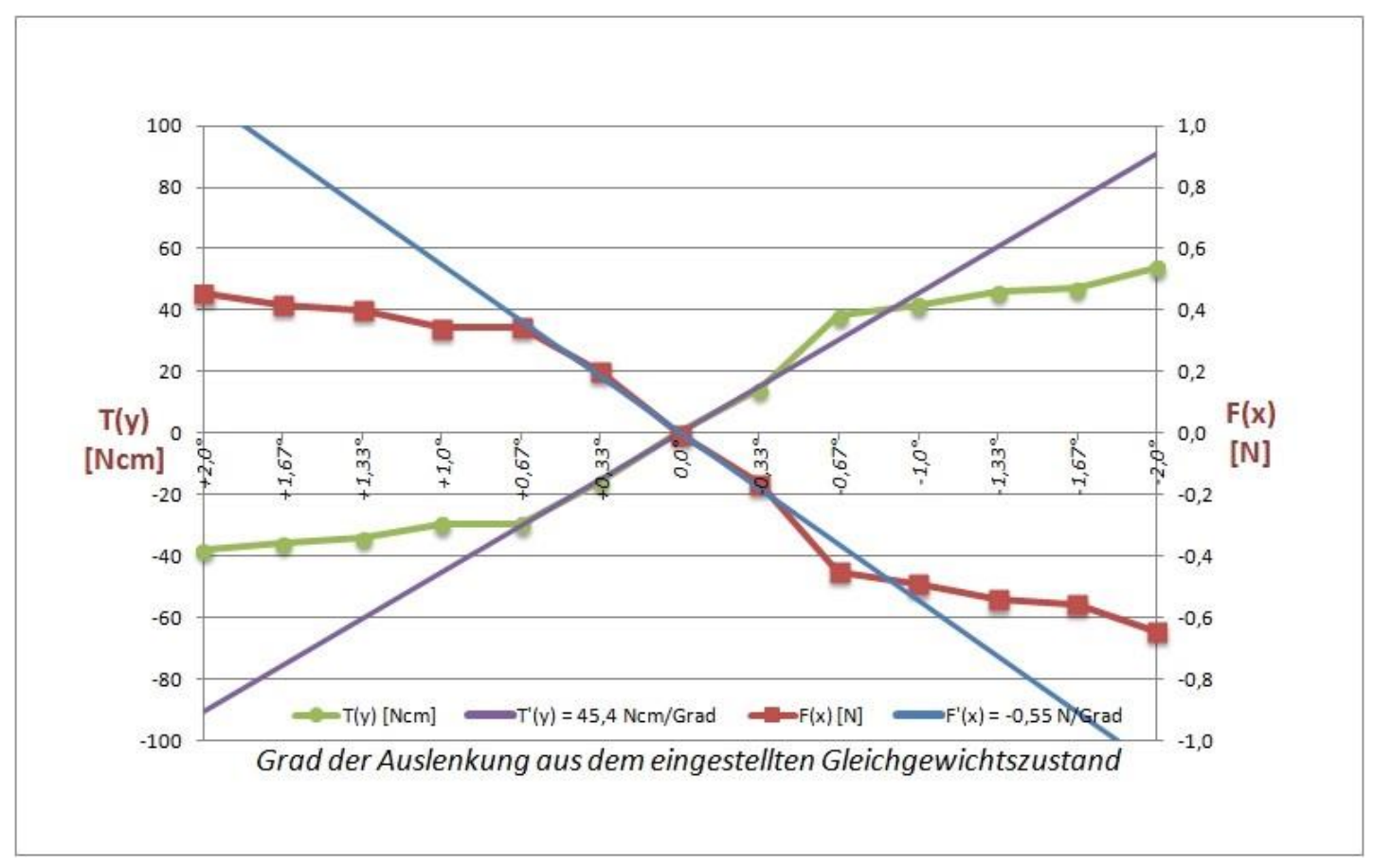

Abbildung 36: Resultierende Kraft und Drehmoment für das AEQUOS-Knie bei Auslenkungen im Flexionswinkel $5^{\circ}$, Messung 2.

Die Messung im Flexionswinkel von $5^{\circ}$ wurde in dieser Variante mit einem Verhältnis Hamstring zu Musculus gastrocnemius von 0,89 durchgeführt, mediale und laterale 
Komponenten gleichbelastet (Tabelle 2 und Tabelle 11).

\begin{tabular}{|l|c|c|c|c|c|c|}
\hline & \multirow{2}{*}{ Quadriceps } & \multicolumn{2}{|c|}{ Hamstring } & \multicolumn{2}{c|}{ Gastrocnemius } & Popliteus \\
\hline & & Medial & Lateral & Medial & Lateral & \\
\hline F (N) & 177,39 & 85,78 & 85,40 & 96,47 & 95,62 & 79,33 \\
\hline
\end{tabular}

Tabelle 2: Appliziertes Kräftesystem bei der Messung 2.

Aus Messung 1 und 2 kann gefolgert werden, dass es eine Abnahme der Stabilität für Kraft und Drehmoment gibt durch die Umverteilung in der beugenden Muskulatur von Hamstring zu Musculus gastrocnemius.

\subsubsection{Messung 5}

Die Gleichgewichtseinstellungen beim AEQUOS-Knie bei Auslenkungen aus dem Flexionswinkel von $5^{\circ}$ mit dieser Kraftvariante waren instabil, mit vergleichbarer Ausprägung der Kräfte in Flexions- und Extensionsrichtung. Bei $+2^{\circ}$ Auslenkung betrug $\mathrm{F}(\mathrm{x})-0,44 \mathrm{~N}$ und $\mathrm{T}(\mathrm{y}) 37 \mathrm{Ncm}$, bei $-2^{\circ}$ wurden $+0,45 \mathrm{~N}$ bzw. $-38,1 \mathrm{Ncm}$ gemessen. Der Stabilitätsgrad ergab 0,08 N/Grad für die Kräfte und -6,59 Ncm/Grad für das Drehmoment (Abbildung 37 und Tabelle 10). Auslenkungen führten zu einem labilen Gleichgewicht. 


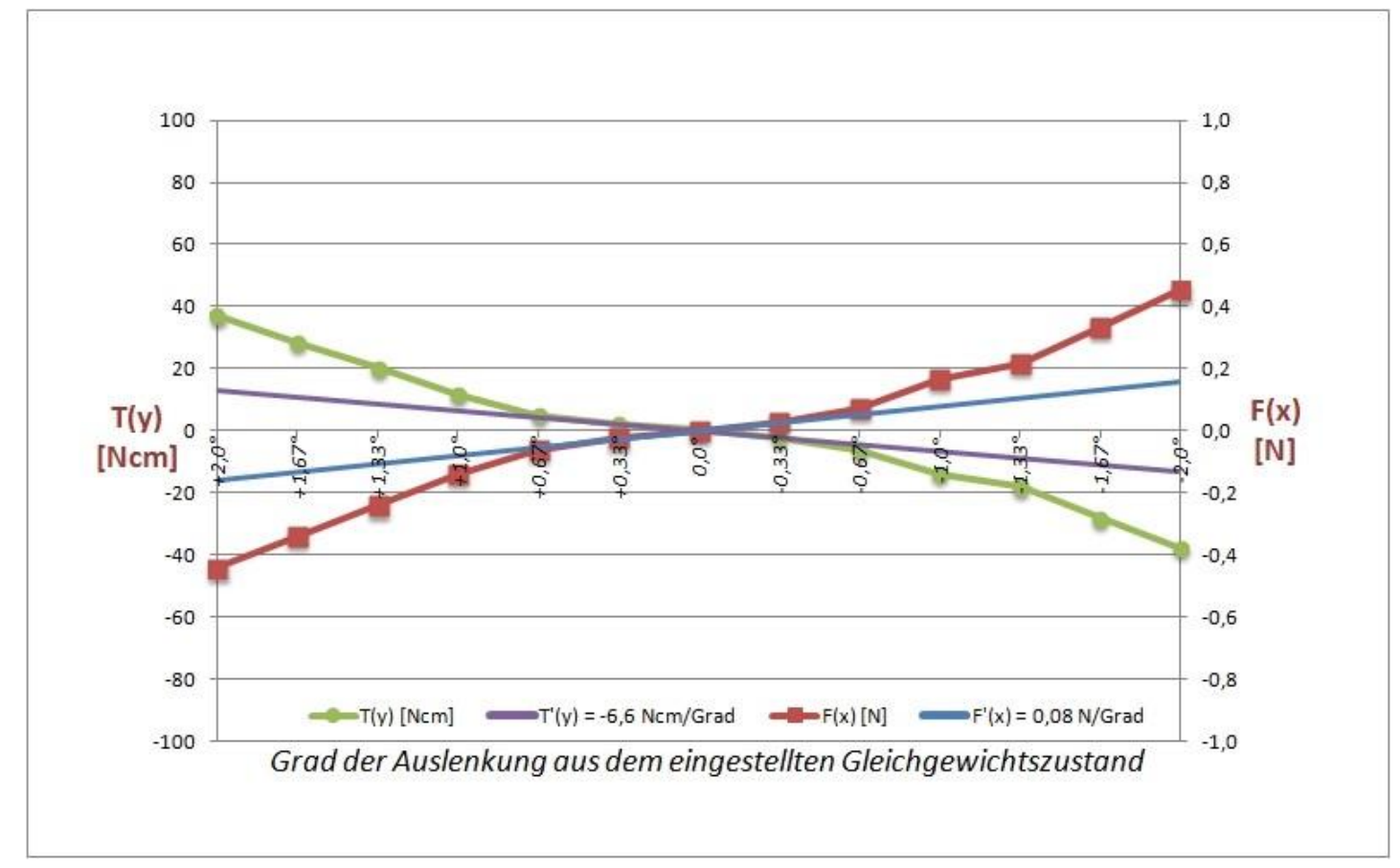

Abbildung 37: Resultierende Kraft und Drehmoment für das AEQUOS-Knie bei Auslenkungen im Flexionswinkel $5^{\circ}$, Messung 5.

Bei der hier gewählten Kraftvariante wurde mit einem Kräfteverhältnis Hamstring zu Musculus gastrocnemius von 0,28 gemessen, mit etwas stärkerer Betonung der medialen Muskelzüge der Musculus gastrocnemius (Tabelle 3 und Tabelle 11).

\begin{tabular}{|l|c|c|c|c|c|c|}
\hline & \multirow{2}{*}{ Quadriceps } & \multicolumn{2}{|c|}{ Hamstring } & \multicolumn{2}{c|}{ Gastrocnemius } & Popliteus \\
\hline & & Medial & Lateral & Medial & Lateral & \\
\hline F (N) & 286,22 & 34,78 & 36,37 & 136,79 & 113,58 & 12,27 \\
\hline
\end{tabular}

Tabelle 3: Appliziertes Kräftesystem bei der Messung 5.

Korrespondierend $\mathrm{zu}$ Messung 1 und 2 ist auch in Messung 5 die Instabilität gekennzeichnet durch eine Dominanz der Kraft des Musculus gastrocnemius gegenüber der Hamstringkraft. 


\subsubsection{Flexionswinkel $90^{\circ}$}

\subsubsection{Messung 6}

Bei der 2. Messreihe an dem AEQUOS-Knie im Flexionswinkel von $90^{\circ}$, mit Einstellung dieser Kraftvariante, wurde eine instabile Gleichgewichtslage im Drehmoment und eine indifferente Gleichgewichtslage im Kraftgleichgewicht durch die Auslenkungen zwischen $+2^{\circ}$ und $-2^{\circ}$ nachgewiesen. Die Instabilität war in Extensionsrichtung etwas stärker. Bei $+2^{\circ}$ betrug $\mathrm{F}(\mathrm{x})-0,31 \mathrm{~N}, \mathrm{~T}(\mathrm{y})$ ergab 25,8 $\mathrm{Ncm}$. Bei $-2^{\circ}$ wurden ein $\mathrm{F}(\mathrm{x})$ von $0,47 \mathrm{~N}$ und ein Drehmoment von $-39,6 \mathrm{Ncm}$ ermittelt. Der Stabilitätsgrad betrug 0,05 N/Grad für die Kräfte und -4,25 Ncm/Grad für das Drehmoment (Abbildung 38 und Tabelle 10).

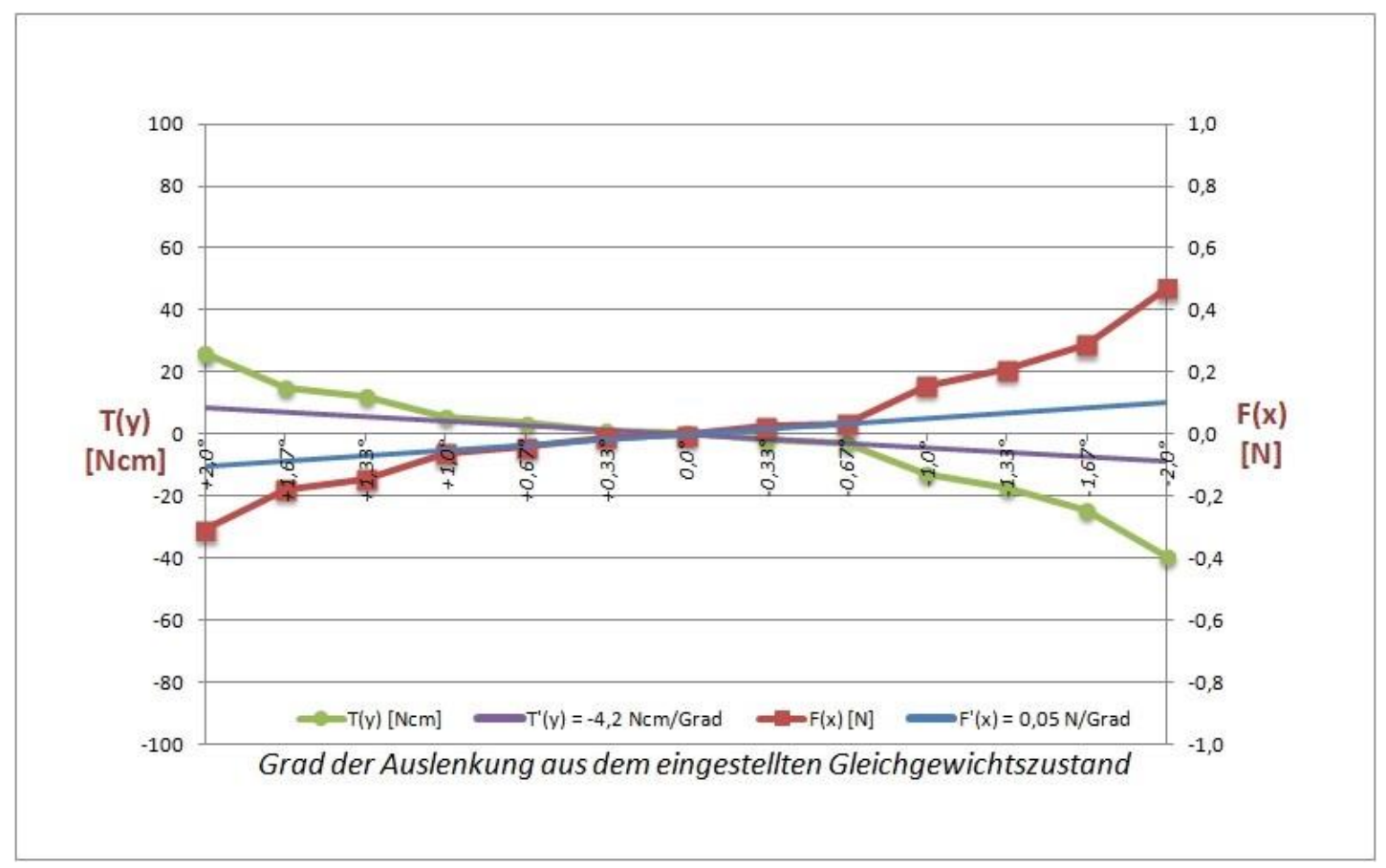

Abbildung 38: Resultierende Kraft und Drehmoment für das AEQUOS-Knie bei Auslenkungen im Flexionswinkel $90^{\circ}$, Messung 6.

Bei dieser Messreihe wurde Hamstring zu Musculus gastrocnemius im Verhältnis 0,41 belastet, wobei mediale und laterale Muskelzüge approximativ gleichbelastet wurden (Tabelle 4 und Tabelle 11). Hierbei wurde das gleiche Kräfteverhältnis verwendet wie bei der Messung 9. 


\begin{tabular}{|c|c|c|c|c|c|c|}
\hline & Quadriceps & \multicolumn{2}{|c|}{ Hamstring } & \multicolumn{2}{c|}{ Gastrocnemius } & Popliteus \\
\hline & & Medial & Lateral & Medial & Lateral & \\
\hline F (N) & 289,3 & 47,9 & 49,0 & 117,2 & 116,7 & 0 \\
\hline
\end{tabular}

Tabelle 4: Appliziertes Kräftesystem bei der Messung 6.

Es zeigt sich eine weniger ausgeprägte Instabilität als in der Messung zuvor, der Musculus gastrocnemius hat eine stärkere Kraft als die Hamstring, aber weniger Kraft als in der Messung zuvor.

\subsubsection{Messung 7}

Die Einstellung einer weiteren Kraftvariante zur Erzielung von Auslenkungen im Flexionswinkel von $90^{\circ}$ führte für das AEQUOS-Knie $\mathrm{zu}$ einer stabilen Gleichgewichtslage. In Extensionsrichtung traten höhere Kräfte auf, so wurde bei $+2^{\circ}$ eine rücktreibende Kraft von 0,27 N gemessen, bei $-2^{\circ}$ dagegen von $-0,79 \mathrm{~N}$. Die Drehmomente betrugen $-22,9 \mathrm{Ncm}$ für $+2^{\circ}$ und $66,7 \mathrm{Ncm}$ für $-2^{\circ}$. Der Stabilitätsgrad betrug -0,32 N/Grad für die Kräfte und 26,52 Ncm/Grad für das Drehmoment (Abbildung 39 und Tabelle 10).

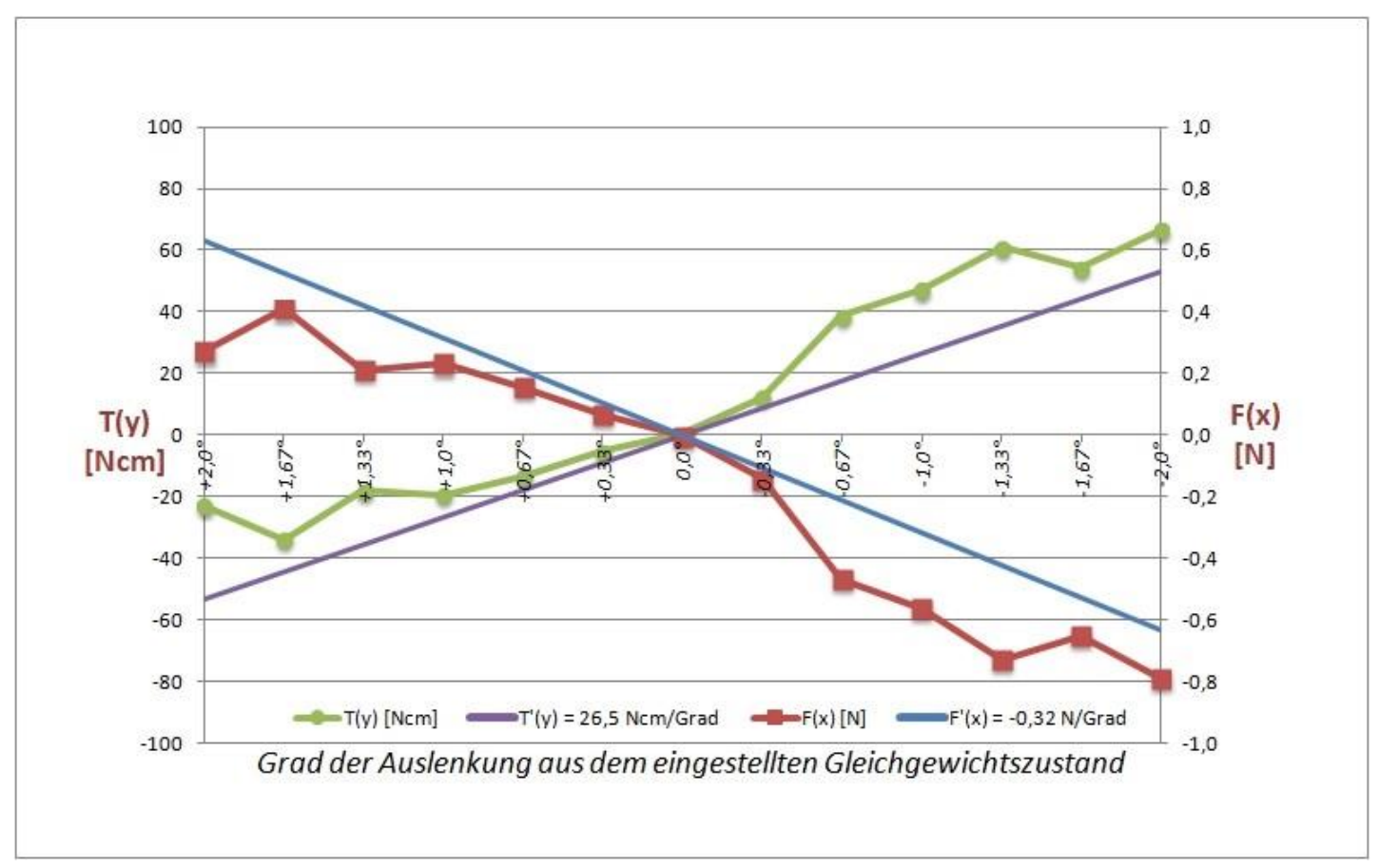

Abbildung 39: Resultierende Kraft und Drehmoment für das AEQUOS-Knie bei Auslenkungen im Flexionswinkel $90^{\circ}$, Messung 7. 
Für diese Versuchsreihe wurde eine Krafteinstellung von Hamstring zu Musculus gastrocnemius von 2,67 vorgegeben, mit etwa gleicher Belastung der medialen und lateralen Muskelzüge (Tabelle 5 und 11).

\begin{tabular}{|l|c|c|c|c|c|c|}
\hline & Quadriceps & \multicolumn{2}{|c|}{ Hamstring } & \multicolumn{2}{c|}{ Gastrocnemius } & Popliteus \\
\hline & & Medial & Lateral & Medial & Lateral & \\
\hline F (N) & 311,0 & 111,9 & 112,9 & 42,0 & 42,2 & 0 \\
\hline
\end{tabular}

Tabelle 5: Appliziertes Kräftesystem bei der Messung 7.

Auch bei dieser Messung führte eine überwiegende Hamstringkraft zu einer stabilen Gleichgewichtslage.

\subsection{Mebio Scan-Knie}

\subsubsection{Flexionswinkel $5 \circ$}

\subsubsection{Messung 3}

Bei der Messreihe zum Mebio Scan-Knie, Flexionswinkel $5^{\circ}$, wurde mit der ersten Kraftvariante ein stabiler Gleichgewichtszustand erreicht, mit einem $\mathrm{F}(\mathrm{x})$ von $-0,02 \mathrm{~N}$ und einem $\mathrm{T}(\mathrm{y})$ von $2,4 \mathrm{Ncm}$ bei einer Auslenkung von $+2^{\circ}$ und einem $\mathrm{F}(\mathrm{x})$ von $0,04 \mathrm{~N}$ und einem $\mathrm{T}(\mathrm{y})$ von $-4,1 \mathrm{Ncm}$ bei $-2^{\circ}$. Der Stabilitätsgrad des stabilen Gleichgewichts betrug -0,11 N/Grad für die Kräfte und 8,31 Ncm/Grad für das Drehmoment (Abbildung 40 und Tabelle 10). 


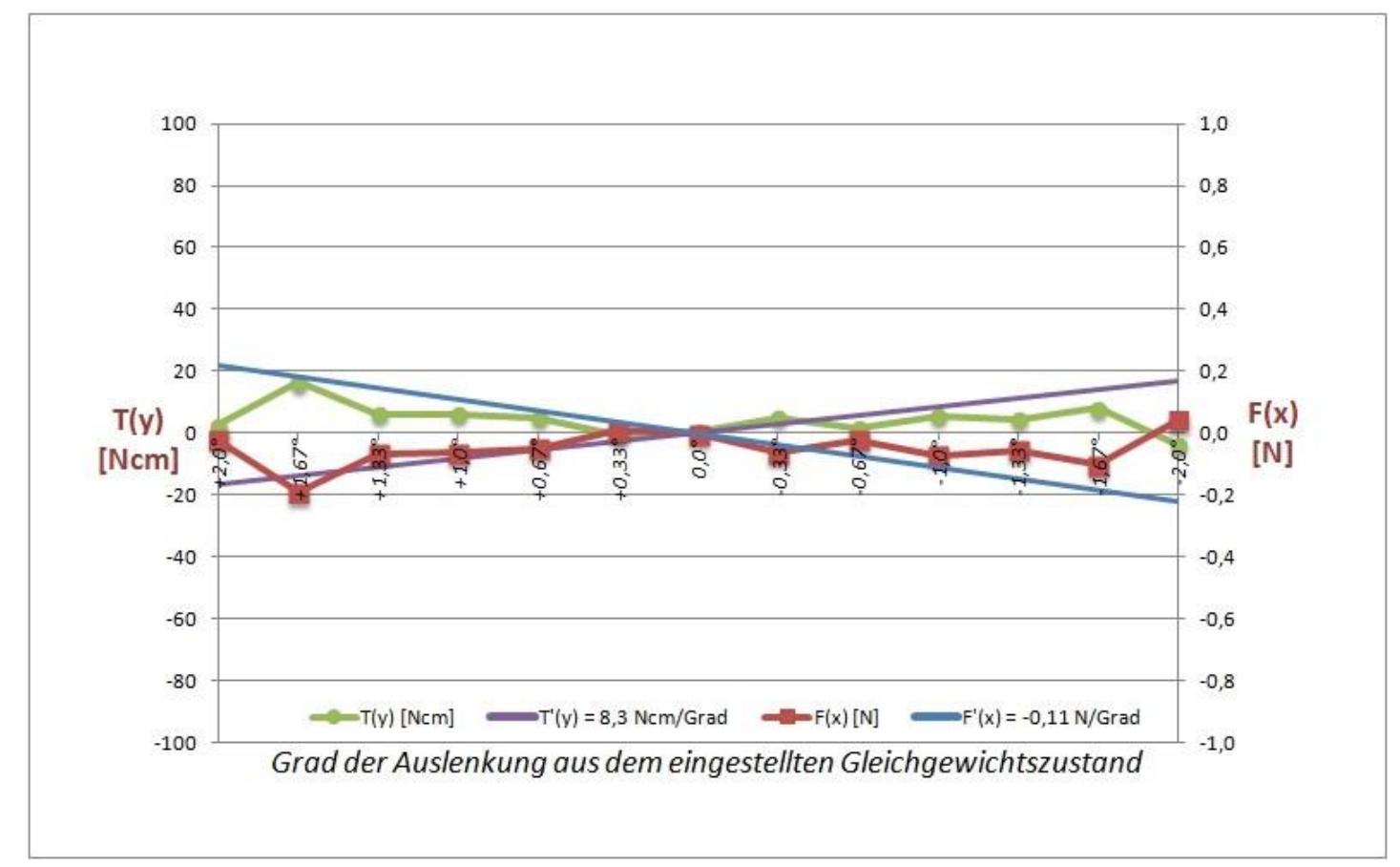

Abbildung 40: Resultierende Kraft und Drehmoment für das Mebio Scan-Knie bei Auslenkungen im Flexionswinkel $5^{\circ}$, Messung 3.

Für die Messung des Mebio Scan-Knie im Flexionswinkel $5^{\circ}$, betrug das Verhältnis Hamstring zu Musculus gastrocnemius 3,04 mit gleicher Belastung der medialen und lateralen Muskelzüge (Tabelle 6 und 11).

\begin{tabular}{|l|c|c|c|c|c|c|}
\hline & Quadriceps & \multicolumn{2}{|c|}{ Hamstring } & \multicolumn{2}{c|}{ Gastrocnemius } & Popliteus \\
\hline & & Medial & Lateral & Medial & Lateral & \\
\hline F(N) & 215,00 & 143,11 & 143,18 & 46,98 & 47,04 & 24,71 \\
& & & & & & \\
\hline
\end{tabular}

Tabelle 6: Appliziertes Kräftesystem bei der Messung 3.

Beim Mebio Scan-Knie wurde in dieser Messung ein geringer stabiler Stabilitätsgrad bei überwiegender Hamstringkraft registriert.

\subsubsection{Kraftvariante Messung 4}

Bei dieser Einstellung zur Messung des Mebio Scan-Knie im Flexionswinkel $5^{\circ}$ 
resultierte ein stabiler Gleichgewichtszustand, mit einem maximalen $\mathrm{F}(\mathrm{x})$ bei $+2^{\circ}$ von $+0,16 \mathrm{~N}$ und einem Drehmoment von $-13,5 \mathrm{Ncm}$, sowie bei $-2^{\circ}$ mit einem $\mathrm{F}(\mathrm{x})$ von 0,35 N und einem Drehmoment von 30,1 Ncm. Der Stabilitätsgrad betrug 0,26 N/Grad für die Kräfte und 21,6 Ncm/Grad für das Drehmoment (Abbildung 41 und Tabelle 10).

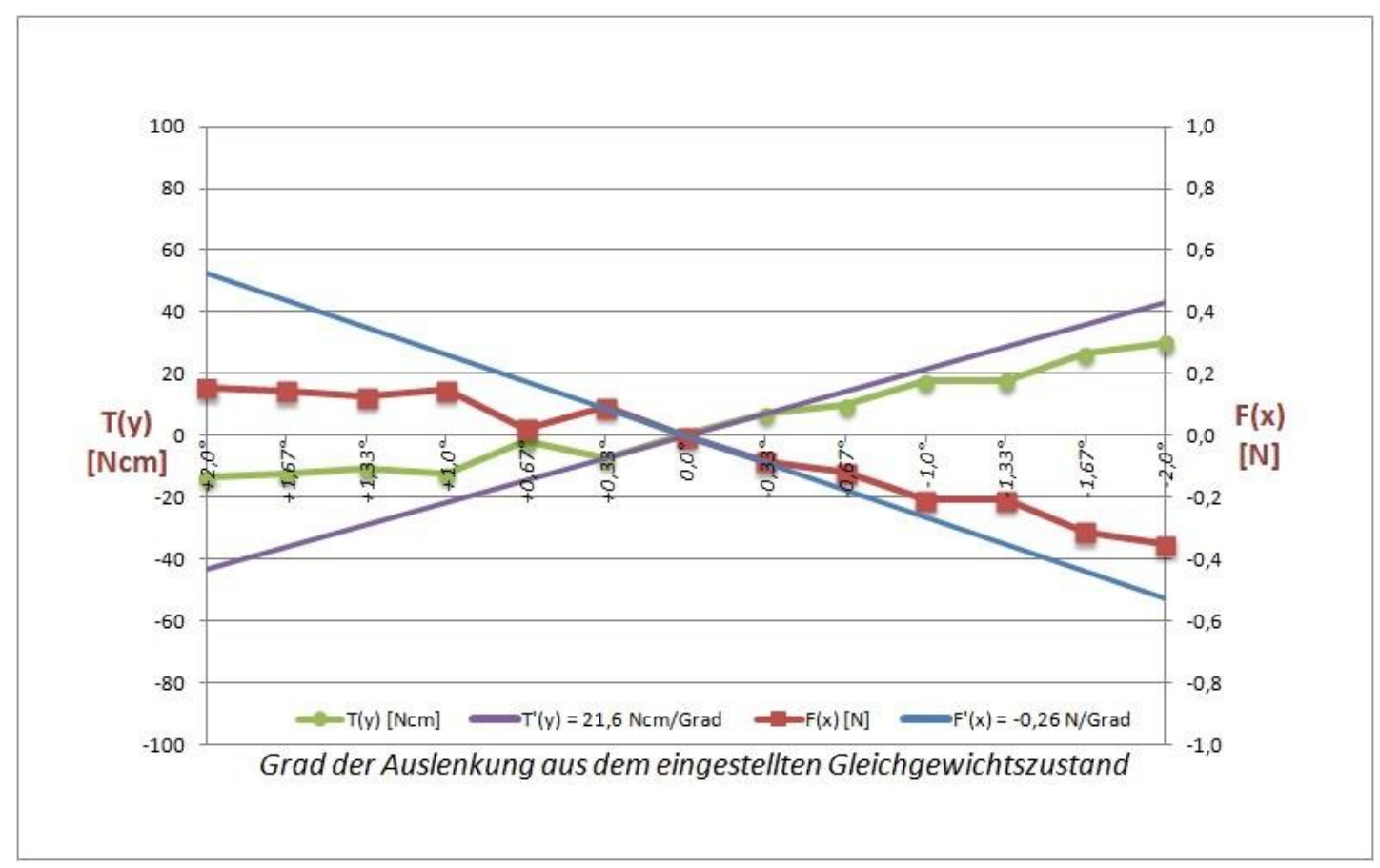

Abbildung 41: Resultierende Kraft und Drehmoment für das Mebio Scan-Knie bei Auslenkungen im Flexionswinkel $5^{\circ}$, Messung 4.

In dieser Kraftvariante zur Messung im Flexionswinkel von $5^{\circ}$ betrug das Verhältnis Hamstring zu Musculus gastrocnemius 0,83, wobei mediale und laterale Muskeln gleichermaßen beteiligt waren (Tabelle 7 und Tabelle 11).

\begin{tabular}{|l|c|c|c|c|c|c|}
\hline & \multirow{2}{*}{ Quadriceps } & \multicolumn{2}{|c|}{ Hamstring } & \multicolumn{2}{c|}{ Gastrocnemius } & Popliteus \\
\hline & & Medial & Lateral & Medial & Lateral & \\
\hline F(N) & 178,3 & 90,2 & 89,9 & 109,4 & 108,4 & 43,8 \\
\hline
\end{tabular}

Tabelle 7: Appliziertes Kräftesystem bei der Messung 4.

Es zeigt sich ein gering ausgeprägter stabiler Gleichgewichtszustand bei Überwiegen der Kraft des Musculus gastrocnemius gegenüber den Hamstring. 


\subsubsection{Flexionswinkel $90^{\circ}$}

\subsubsection{Messung 8}

Die Auslenkungen aus dem Flexionswinkel von $90^{\circ}$ führte beim Mebio Scan-Knie zu einer stabilen Gleichgewichtseinstellung. Die rücktreibende Kraft betrug 0,54 $\mathrm{N}$ bei $+2^{\circ}$ und $-0,81 \mathrm{~N}$ bei $-2^{\circ}$. Die Drehmomente lagen bei $-45,9 \mathrm{Ncm}$ bei $+2^{\circ}$ und $68,0 \mathrm{Ncm}$ bei $-2^{\circ}$. Der Stabilitätsgrad betrug $-0,21 \mathrm{~N} / \mathrm{Grad}$ für die Kräfte und $17,1 \mathrm{Ncm} / \mathrm{Grad}$ für das Drehmoment bei einem stabilen Gleichgewicht (Abbildung 42 und Tabelle 10).

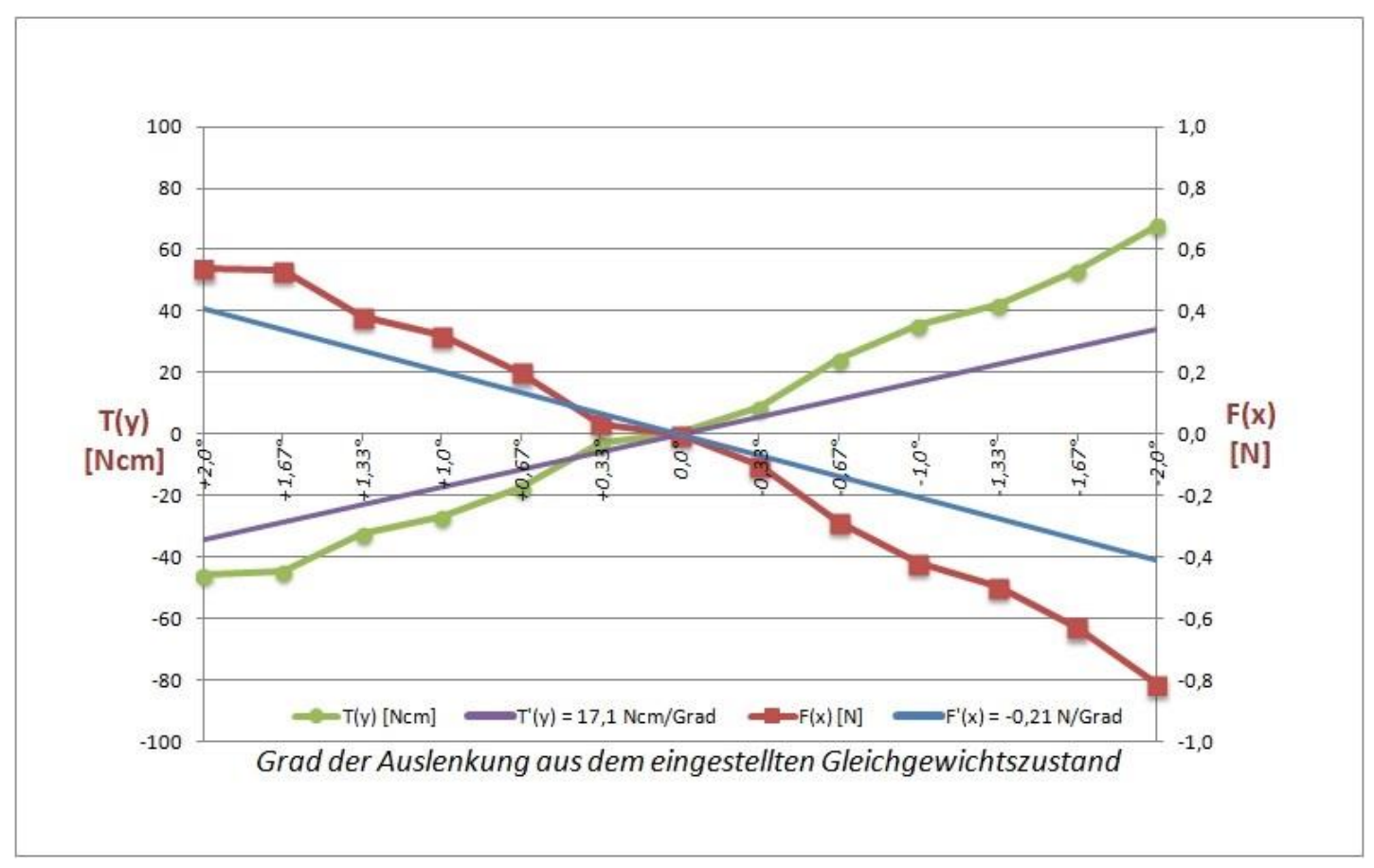

Abbildung 42: Resultierende Kraft und Drehmoment für das Mebio Scan-Knie bei Auslenkungen im Flexionswinkel $90^{\circ}$, Messung 8.

Für die Einstellung dieser Kraftkomposition zur Messung im Flexionswinkel von $90^{\circ}$ wurde das Verhältnis 1,15 Hamstring zu Musculus gastrocnemius gewählt, mit gleicher Belastung der medialen und lateralen Muskelzüge (Tabelle 8 und Tabelle 11).

\begin{tabular}{|l|c|c|c|c|c|c|}
\hline & \multirow{2}{*}{ Quadriceps } & \multicolumn{2}{|c|}{ Hamstring } & \multicolumn{2}{c|}{ Gastrocnemius } & \multirow{2}{*}{ Popliteus } \\
\hline & & Medial & Lateral & Medial & Lateral & \\
\hline F(N) & 333,5 & 82,3 & 70,7 & 66,6 & 66,9 & 0 \\
\hline
\end{tabular}

Tabelle 8: Appliziertes Kräftesystem bei der Messung 8. 
Die deutlich stabile Gleichgewichtseinstellung zeigt im Vergleich zur vorherigen Messung eine Reduktion der Kraft des Musculus gastrocnemius.

\subsubsection{Messung 9}

In der Einstellung mit dieser Kraftvariante beim Flexionswinkel von $90^{\circ}$ wurde für die Mebio Scan-Endoprothese in Flexionsrichtung eine gering ausgeprägte stabile Gleichgewichtslage erreicht, mit einer maximalen Kraft von $-0,11 \mathrm{~N}$ und einem Drehmoment von 8,9 $\mathrm{Ncm}$ bei einer Auslenkung von $+2^{\circ}$. Bei der Auslenkung in Extensionsrichtung stellte sich ein stabiler Zustand ein, mit einem $\mathrm{F}(\mathrm{x})$ von $-0,16$ und einem $\mathrm{T}(\mathrm{y})$ von 13,3 bei einer Auslenkung von $-2^{\circ}$. Der Stabilitätsgrad betrug 0,26 N/Grad für die Kräfte und 21,2 Ncm/Grad für das Drehmoment (Abbildung 43 und Tabelle 10).

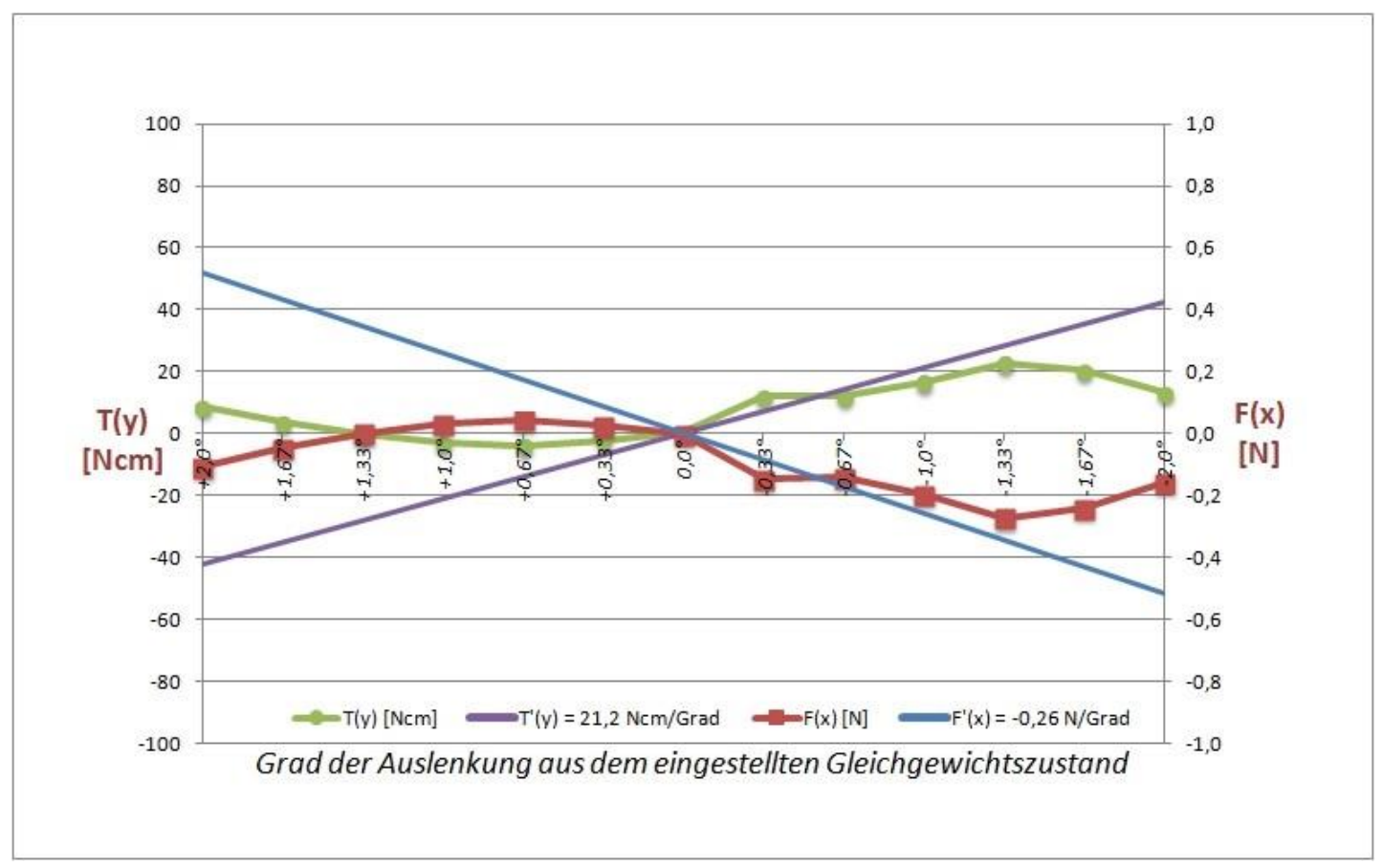

Abbildung 43: Resultierende Kraft und Drehmoment für das Mebio Scan-Knie bei Auslenkungen im Flexionswinkel $90^{\circ}$, Messung 9.

In dieser Kraftvariante bei $90^{\circ}$ wurde mit einem Kräfteverhältnis Hamstring zu Musculus gastrocnemius von 0,41 gearbeitet, auf mediale und laterale Kräfte gleichermaßen verteilt (Tabelle 9 und 11). 


\begin{tabular}{|l|c|c|c|c|c|c|}
\hline & \multirow{2}{*}{ Quadriceps } & \multicolumn{2}{|c|}{ Hamstring } & \multicolumn{2}{c|}{ Gastrocnemius } & \multirow{2}{*}{ Popliteus } \\
\hline & & Medial & Lateral & Medial & Lateral & \\
\hline F(N) & 289,3 & 47,9 & 49,0 & 117,2 & 116,7 & 0 \\
\hline
\end{tabular}

Tabelle 9: Appliziertes Kräftesystem bei der Messung 9.

Diese gering stabil ausgeprägte Stabilitätslage weist eine Erhöhung der Kraft des Musculus gastrocnemius auf, die deutlich die Kraft der Hamstring überwiegt.

\subsection{Vergleich der Stabilitätsgrade}

Für die Darstellung der Beeinflussung des Stabilitätsgrades durch die Diversität der Muskelkombinationen wurde in dem folgenden Graphen (Abbildung 44) das erzeugte Drehmoment T`(y) gegen die Muskelverhältnisse von den Hamstrings zum Musculus gastrocnemius aufgetragen.

Dabei entsprechen die Punkte

A1 = Stabilitätsgrad T` ${ }^{\prime}$ y) der Messung 1 AEQUOS-Knie bei $5^{\circ}$ (Vergleiche Tabelle 11 im Anhang),

A2 = Stabilitätsgrad T ${ }^{`}(\mathrm{y})$ der Messung 2 AEQUOS-Knie bei $5^{\circ}$

M3 = Stabilitätsgrad T`(y) der Messung 3 Mebio Scan-Knie bei $5^{\circ}$

M4 = Stabilitätsgrad T`(y) der Messung 4 Mebio Scan-Knie bei $5^{\circ}$

A5 = Stabilitätsgrad T ${ }^{`}(\mathrm{y})$ der Messung 5 AEQUOS-Knie bei $5^{\circ}$

A6 = Stabilitätsgrad T`(y) der Messung 6 AEQUOS-Knie bei $90^{\circ}$

A7 = Stabilitätsgrad T` $(y)$ der Messung 7 AEQUOS-Knie bei $90^{\circ}$

M8 = Stabilitätsgrad T`(y) der Messung 8 Mebio Scan-Knie bei $90^{\circ}$

M9 = Stabilitätsgrad T`(y) der Messung 9 Mebio Scan-Knie bei $90^{\circ}$ 


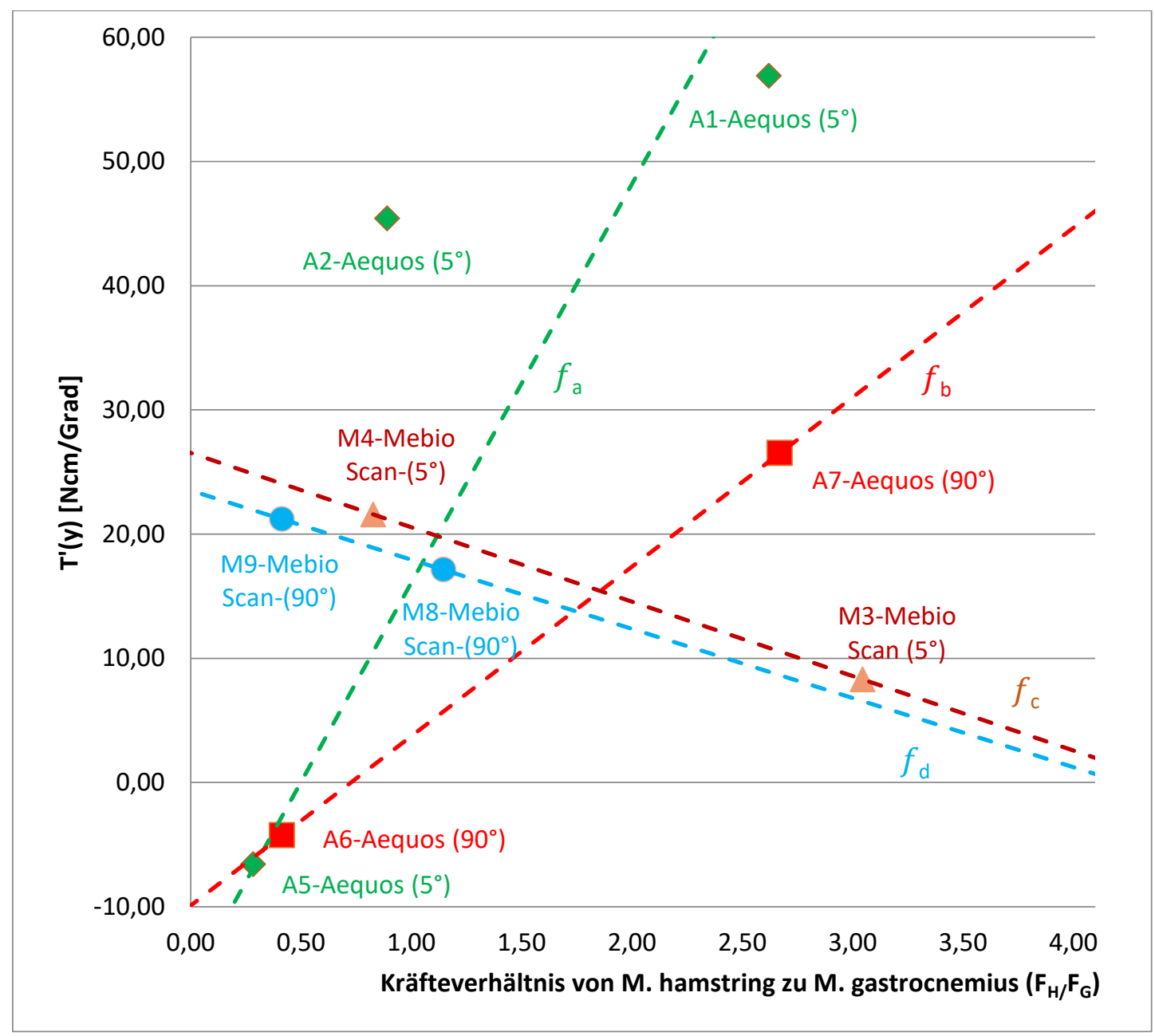

Abbildung 44: Zusammenhang zwischen dem Kräfteverhältnis der Flexoren Hamstring zu Musculus gastrocnemius (Abszisse) und dem erzeugtem Drehmoment T'(y)in Ncm/Grad (Ordinate).

Es zeigt sich, dass mit dem AEQUOS-Knie bei $5^{\circ}$ sowohl stabile (A1/A2) als auch instabile Gleichgewichtslagen im Drehmoment auftreten (A5).

Beim Mebio Scan-Knie konnten hingegen nur stabile Gleichgewichtslagen im Drehmoment bei $5^{\circ}$ erzeugt werden (M3/M4).

Des Weiteren führt ein stärkeres Verhältnis von den Hamstrings zum Musculus gastrocnemius beim AEQUOS-Knie $\mathrm{zu}$ deutlich ausgeprägteren stabilen Gleichgewichtslagen im Drehmoment.

Bei $90^{\circ}$ ließ sich beim AEQUOS-Knie eine stabile (A7) und eine instabile (A6) Gleichgewichtslage auffinden, beim Mebio Scan-Knie hingegen erneut nur stabile (M8/M9).

Beim AEQUOS-Knie hängt der Stabilitätsgrad $T^{`}(y)$ vom Kraftverhältnis der flektierenden Muskeln Hamstring - Musculus gastrocnemius ab. 
Dabei zeigt sich, dass das Gleichgewicht des Flexion-/Extensionsdrehmoments umso stabiler wird, je mehr die Kraft der Hamstrings dominiert. Dies gilt sowohl für die Flexion von $5^{\circ}$ (Kurve a) als auch für die Flexion von $90^{\circ}$ (Kurve b).

Bei der Mebio Scan-Endoprothese konnten in allen Messungen nur stabile Positionen gefunden werden. Hierbei zeigte sich, dass je mehr die Flexionskraft der Hamstrings die des Musculus gastrocnemius überwiegt, umso kleiner wurde der stabile Stabilitätsgrad (Kurve c und d). 


\section{Diskussion}

\subsection{Messsystem}

Die vorliegende Arbeit hatte zum Ziel, die mechanische Stabilität von Knieendoprothesen $\mathrm{zu}$ untersuchen. Über unterschiedliche Kombinationen von Muskelkräften bei vorgegeben Knie-Flexionswinkeln konnten Gleichgewichtslagen eingestellt werden. Es wurde sowohl für das AEQUOS-Knie als auch für das Mebio Scan-Knie das Ausmaß der Stabilität beziehungsweise der Instabilität bei vorgegebenen Gleichgewichten untersucht.

Mit der modifizierten Apparatur war es möglich, die Stabilität beziehungsweise die Instabilität einer Gleichgewichtslage messtechnisch zu erfassen. Durch eine Änderung der Beträge der Muskelkräfte waren unterschiedliche Ausmaße der Gleichgewichte bei $5^{\circ}$ beziehungsweise $90^{\circ}$ Kniebeuge möglich, da sechs Muskelkräfte bei vier kinematischen Freiheitsgraden vorlagen. Bei der Messung wurde eine äußere Zwangsführung der Artikulationsflächen vermieden, der Kontakt wurde unter Freischaltung aller Freiheitsgrade ausschließlich unter Kraftschluss hergestellt. Kraftschluss konnte stattfinden, indem über sechs simulierte Muskelzüge unterschiedliche Kombinationen von Muskelkräften der Muskelgruppen Musculus quadriceps, der Flexoren- oder Hamstringgruppe (medial und lateral) sowie des Musculus gastrocnemius (medial und lateral) und des Musculus popliteus eingestellt wurden.

Mit der Messanordnung wurde erreicht, die Wirkungsrichtung der Kraftvektoren nach den anatomischen Vorgaben der das Kniegelenk beeinflussenden Muskulatur nachzuempfinden. Hierzu wurden die physiologischen Ansatzpunkte der Muskeln in der Apparatur nachgestellt.

Die vorgestellte Messapparatur kann richtungsweisende Ergebnisse liefern. Da sie als Vergleichsmessungen ausgelegt sind, können sie als ausreichend für die Beurteilung der Statik des Tibiofemoralgelenks einer Knie-Endoprothese erachtet werden.

Von besonderem Interesse waren auch der Roll-Gleit-Vorgang und die Beobachtung, wie bei den beiden ausgewählten Endoprothesen diese physikalische Anforderung gelöst wird. Eine Knie-Endoprothese muss nicht nur Gang und Stand, sondern auch alle übrigen Bewegungen und Positionen mit Beugung des Knies wie beim Aufstehen aus der Hocke oder Treppensteigen im Alltagsleben gewährleisten, daher wurden 
Flexionswinkel von $5^{\circ}$ und $90^{\circ}$ für die Gleichgewichtslagen ausgewählt. Da hier labile Gleichgewichtslagen für die dynamischen Übergänge im Bewegungsablauf erforderlich sind.

Mit der vorliegenden Arbeit konnte bestätigt werden, dass das Tibiofemoralgelenk des AEQUOS-Knies in der Lage ist, bei vorgegebenen Flexionswinkeln sowohl stabile als auch instabile Gleichgewichtszustände einzunehmen (Abbildung 44). Unterschiedliche Gleichgewichtszustände konnten durch Einstellung unterschiedlicher Kombinationen vor allem der beugenden Muskelkräfte erzeugt werden.

Eine wesentliche Modifikation der in den Arbeiten von Stühmer und Knösel verwendeten Apparatur war der Wegfall aller festen Verbindungen zwischen Tibia und Femur sowie eine Gewichtsreduzierung der gesamten Apparatur. Auf diese Weise wurde es möglich, die Fehlerquellen, die durch Zwangsführung und Erschütterungen zu Kontaktpunktänderungen führen können, auszuschalten. Ebenso war es mit Hilfe der modifizierten Kalibrierung, wobei nun in zwei Raumrichtungen kalibriert werden konnte, möglich, die Fehler der bisher nur in $+x$-Richtung aufgenommenen Messwerte zu vermeiden.

So war sichergestellt, dass alle Freiheitsgrade des Kniegelenks bei der Gleichgewichtseinstellung genutzt werden konnten.

\subsection{Muskelkräfte und Flexionswinkel}

Die in der vorliegenden Arbeit betrachteten Flexionswinkel des Kniegelenks von $5^{\circ}$ und $90^{\circ}$ wurden gewählt, weil sie zwei bedeutsame Winkel für Alltagssituationen darstellen.

Bei $5^{\circ}$-Knieflexion beginnt der Gangzyklus und endet die mittlere Stützphase beim Vorgang des Gehens (Abbildung 12). Beim Gehen sind labile und stabile Gleichgewichtszustände des Knies wichtig. Dies gilt auch für Endoprothesen in ihrer kinematischen Funktion.

Im Bereich von $5^{\circ}$ konnten beim AEQUOS-Knie in zwei unterschiedlichen Kraftvarianten unterschiedliche Gleichgewichte sowohl im Kraftgleichgewicht als auch im Drehmomentgleichgewicht eingestellt werden (Tabelle 10 und Abbildung 44). Ein labiler Zustand in Kraft und Drehmoment in der Messung 5 konnte durch eine Dominanz des Musculus gastrocnemius realisiert werden. Auch in der $90^{\circ}$ - 
Flexionsstellung (6) konnte durch hohe Kräfte des Musculus gastrocnemius ein gering instabiler Zustand im Drehmoment und Kraftgleichgewicht registriert werden. In beiden Beugelagen wird der Stabilitätsgrad durch das Verhältnis der Kräfte von Hamstring zu Gastrocnemiusmuskulatur eingestellt: Zunehmender Anteil der Kraft der Hamstringmuskulatur, um die extendierende Kraft des Musculus quadriceps zu kompensieren, erhöht den Stabilitätsgrad.

$90^{\circ}$-Flexionswinkel werden notwendigerweise bei Vorgängen wie Treppensteigen oder beim Aufheben eines Gegenstands vom Boden eingenommen. Auch hier ist es wichtig, sowohl stabile als auch labile Gleichgewichte beim gleichen Flexionswinkel realisieren zu können, damit aus einer stabilen Position dynamische Bewegungen hervorgehen können wie beim Aufstehen aus der Hocke. Daher ist es von entscheidender Bedeutung, dass in Messung 6 ein instabiles Gleichgewicht im Drehmoment beim AEQUOS-Knie vorliegt bei Dominanz der Kraft des Musculus gastrocnemius.

Die Messreihen 6 (AEQUOS-Knie $90^{\circ}$ ) und 9 (Mebio Scan-Knie $90^{\circ}$ ) wurden mit der gleichen Kräfteverteilung durchgeführt (Tabelle 10) und führten beim AEQUOSKnie zu einem instabilen Gleichgewicht im Drehmoment und beim Mebio Scan-Knie $\mathrm{zu}$ einem stabilen Gleichgewicht. Dieses Ergebnis verdeutlicht, dass das Prothesendesign das Gleichgewicht beeinflusst. Beim Mebio Scan-Knie konnte im $90^{\circ}$-Flexionswinkel kein labiles Gleichgewicht eingestellt werden, trotz zahlreicher unterschiedlicher applizierter Kraftsysteme. Ein labiles Gleichgewicht ist im $90^{\circ}$ Flexionswinkel von großer Bedeutung für das Aufstehen aus der Hocke, da das Aufstehen ohne Unterstützung nur stattfinden kann, wenn ein labiles Gleichgewicht im Drehmoment vorliegt, welches beim AEQUOS-Knie vorlag. Somit sollte ein Patient mit AEQUOS-Knie alleine durch seine Muskelkraft aufstehen können, wohingegen er beim Mebio Scan-Knie die Hände zur Unterstützung bräuchte. Das AEQUOS-Knie zeigt bei größerer Kraft des Musculus gastrocnemius eine instabile Gleichgewichtsposition im $90^{\circ}$-Kniewinkel, aus der nun ein rein muskulär erzeugtes Aufstehen erzeugt werden kann, sobald ein Extensionsdrehmoment, erzeugt durch den Musculus gastrocnemius, auftritt und das Kniegelenk erneut eine stabile Position einnimmt. Ertastet man beim Aufstehen aus der Hocke den eigenen Musculus gastrocnemius, so bemerkt man, dass dieser beim Aufstehen als erster kontrahiert. 
Hier zeigte das AEQUOS-Knie die besonderen Vorteile der Asymmetrie der Rotationsmittelpunkte der Gelenkflächen: Es konnten zwei unterschiedliche Gleichgewichtszustände eingestellt werden, wobei das labile Gleichgewicht unter hohem Kräfteverhältnis des Musculus gastrocnemius im Vergleich zu der HamstringGruppe, wie auch bei $5^{\circ}$ (Tabelle 11), evoziert wurde. Stabilität im $90^{\circ}$-Winkel wurde unter stärkerer Einwirkung des Musculus quadriceps und im Vergleich zum Musculus gastrocnemius stärkerer Beteiligung der Hamstrings erreicht.

Beim AEQOUS-Knie lagen sowohl instabile als auch stabile Gleichgewichtspositionen vor in den Kniewinkeln von $5^{\circ}$ und $90^{\circ}$, wobei das Gleichgewicht umso stabiler wird je mehr die Kraft der Hamstrings gegenüber dem Musculus gastrocnemius überwiegt.

Beim Mebio Scan-Knie stellt es sich anders dar. Es wurden nur stabile Positionen registriert, und die Stabilität nahm ab bei Überwiegen der Hamstring zum Musculus gastrocnemius.

\subsection{Vergleich von AEQUOS-Knie und Mebio Scan-Knie}

Da das menschliche Kniegelenk kein Scharniergelenk darstellt, sondern durch die Inkongruenz der Gelenkflächen und den Versatz der femoralen Achsen in seiner Funktion bestimmt wird, sind qualitativ unterschiedliche Stabilitäts- und Instabilitätslagen möglich.

Dies ist durch die vorliegenden Ergebnisse bei der AEQUOS-Endoprothese, die nach dem natürlichen Vorbild gestaltet wurde, bewiesen worden.

Bei dem Mebio Scan-Knie gestaltete sich jedoch bereits die Kontaktpunkteinstellung schwierig. Schon vor der Durchführung der Messungen zeigte sich bei dem Mebio Scan-Knie eine nicht gewollte Hyperextension des Tibiofemoralgelenks mit einem unkontrollierten Verschieben der Kontaktpunkte nach ventral. Die Positionierung erwies sich als kompliziert, weil dem Herausgleiten der Tibiakomponente aus dem Femurlager mehrfach entgegengewirkt werden musste.

Eine spontane Selbststabilisierung des Gelenks wie beim AEQUOS-Knie war bei dem Mebio Scan-Knie nicht möglich. Ebenso konnte der notwendige Rollvorgang, der insbesondere beim Flexionswinkel von $5^{\circ}$ wichtig ist, bei dem Mebio Scan-Knie nicht 
erreicht werden. Stattdessen fand eher ein Gleiten/Rutschen der artikulierenden Endoprothesenoberflächen statt.

Im realen Alltagsablauf bedeutet dies zunächst, dass grundsätzlich bei jedem Schritt höhere Kräfte erforderlich sind, um einen einfachen Einkaufs- oder Spaziergang zu absolvieren. Langfristig muss bei einer Endoprothese, die den Rollvorgang nicht korrekt nachvollziehen kann, mit verstärktem Materialabrieb gerechnet werden.

Der bei $90^{\circ}$ gemessene geringere Kraftaufwand der Flexoren bei dem Mebio ScanKnie gegenüber dem AEQUOS-Knie zur Einstellung eines stabilen Gleichgewichts und die Unmöglichkeit, labile Gleichgewichtszustände zu erzielen, unterstützt die Beobachtung, dass mit dem Mebio Scan-Knie dynamische Bewegungsabläufe schwieriger durchzuführen sind.

Die beiden in der vorliegenden Arbeit untersuchten Endoprothesen unterscheiden sich vor allem in ihrer unterschiedlichen geometrischen Gestaltung sowohl des tibialen Plateaus als auch der femoralen Kondylen.

Dies bedingt ein unterschiedliches biomechanisches Verhalten in Bezug auf die Qualität und Quantität von physikalischen Gleichgewichtszuständen bei vorgegebenen Flexionswinkeln und Muskelkräften.

So wurde die AEQUOS-Knie Endoprothese nach der geometrischen Form der natürlichen artikulierenden Flächen des Kniegelenks konzipiert. Die Berücksichtigung der Inkongruenz der Gelenkflächen und der Tatsache, dass das natürliche Kniegelenk ein Viergelenk darstellt, kann allein dem natürlichen Bewegungsablauf gerecht werden, wie auch die hier vorliegenden Messungen gezeigt haben.

Die Eigenschaften des AEQUOS-Knies und der Unterschied der beiden verglichenen Prothesen lassen sich auf die morphologische Gestaltung zurückführen. So lehnen sich bei dem AEQUOS-Knie die Kurvaturen der künstlichen Gelenkfläche an die natürlichen Krümmungen der knöchernen Gelenkflächen an.

Das Mebio Scan-Knie weist keinen Versatz der femoralen Achsen auf und keine beidseitige konkave Gestaltung der beiden tibialen artikulierenden Flächen. Daraus resultiert eine dimere Kette mit zwei Freiheitsgraden in der Flexions/Extensionsbewegung. Um bei dieser dimeren Kette ein Gleichgewicht zu erzeugen, muss die durch die Muskulatur erzeugte Kraftvektorlinie durch die beiden Drehachsen verlaufen. Dies ist gleichwohl schwieriger zu realisieren als beim AEQUOS-Knie, 
welches funktionell in der Flexion/Extension ein zwangsläufiges Viergelenk mit einem Freiheitsgrad darstellt. Hier muss die Kraftwirkungslinie für ein Gleichgewicht lediglich durch die momentane Drehachse laufen. Es ist sehr viel wahrscheinlicher, dass die durch die sechs Muskelkräfte erzeugte Wirkungslinie der resultierenden Kraft die momentane Drehachse schneidet, als dass sie beide Drehachsen des Mebio ScanKnies durchläuft.

Zusammenfassend kann gesagt werden, dass mit dem AEQUOS-Knie, welches dem natürlichen Kniegelenk in seiner Form der artikulierenden Gelenkflächen, insbesondere der Asymmetrie des medialen und lateralen Kompartimentes, nachempfunden ist, durch Variation des muskulären Kraftvektors stabile und instabile Gleichgewichtslagen vor allem im Drehmoment erzeugt werden können und auch das natürlichen Roll-Gleit-Verhalten des Tibiofemoralgelenks auftritt, so dass die von Nägerl 1993 (Nägerl et al. 1993) postulierte Funktionsweise des Tibiofemoralgelenks gestützt werden kann. 


\section{Zusammenfassung}

Die Funktion des natürlichen Kniegelenks ist bestimmt durch die morphologische Gestaltung seiner artikulierenden Flächen von Femur und Tibia. Dabei entstehen unter Einwirkung einer kompressiven Kraft zwei Gelenkkontakte. Die femoralen Gelenkflächen sind medial und lateral konvex geformt, die tibialen Gelenkflächen weisen hingegen medial eine konkave und lateral eine konvexe Krümmung auf. Hieraus ergeben sich entscheidende kinematische Eigenschaften des Kniegelenks in seiner Hauptfunktionsrichtung der Flexion-/Extensionsrichtung. Das Kniegelenk besitzt vier Freiheitsgrade, wird aber von einem muskulären Kraftsystem beeinflusst, das durch die Vielzahl der angreifenden Muskeln sechs Freiheitsgrade besitzt. Dies führt dazu, dass in jeder Gelenkstellung eine Gleichgewichtslage hergestellt werden kann und diese zusätzlich hinsichtlich ihrer Stabilität und Instabilität beeinflusst werden kann. Dies ist notwendig, um sowohl statische (zum Beispiel hocken) als auch kinematische (zum Beispiel aufstehen von einem Stuhl) alltägliche Bewegungsabläufe realisieren zu können (Nägerl et al. 1993). Das Kniegelenk zeigt in der Funktion ein Rollen unter starker Gewichtsbelastung zu Beginn des Gangzyklus, wodurch die Reibung und damit der Verschleiß im Gelenk reduziert werden kann. Diese Eigenschaften des Kniegelenks sollten sich in Knieendoprothesen wiederfinden (Fanghänel et al. 2003, Kapandji 2009, Nägerl et al. 2008).

In dieser Arbeit wurden in einem Messverfahren das AEQUOS-Knie, dessen morphologische Gestaltung der artikulierenden Oberflächen sich an der des natürlichen Tibiofemoralgelenks orientiert, und das Mebio Scan-Knie, welches symmetrisch geformte femorale und tibiale Kondylen und keinen Versatz der femoralen Achsen aufweist, miteinander verglichen.

Die tibialen und fermoralen Endoprothesenkomponenten wurden auf Aluminiumprofile montiert, die Femur und Tibia repräsentierten, und an denen die das Kniegelenk beeinflussende Muskulatur, simuliert über Eisengewichte, angreifen konnte.

Die Apparatur war so gestaltet, dass alle Freiheitsgrade frei geschaltet wurden und eine Zwangsführung ausgeschlossen wurde. Das Femur war starr an einem Kreisbogen fixiert, die Tibia wurde dazu kraftschlüssig alleine über die simulierte angreifende Muskulatur in einer Gleichgewichtslage positioniert. Über eine Kraftmessdose, die am kaudalen Ende der Tibia fixiert wurde, konnte das entstehende Kraftsystem bei kleinen Auslenkungsschritten von $0,33^{\circ}$ gemessen werden. Gemessen wurde in Ausgangs- 
winkeln von $5^{\circ}$ und $90^{\circ}$.

Es zeigte sich während der Messungen, dass das AEQUOS-Knie eine Selbststabilisierung und Rollen aufweist. Die Positionierung des Mebio Scan-Knies erwies sich als schwierig, da es keine definitive Kontaktpunktzuordnung zu geben schien und die Tibia oft in Form einer Hyperextension abglitt.

Beim Mebio Scan-Knie konnten in $5^{\circ}$ und $90^{\circ}$ Flexionswinkel stabile Gleichgewichtszustände festgestellt werden, wobei die Stabilität quantitativ abnahm bei Überwiegen der Hamstring zu Musculus gastrocnemius.

Das AEQUOS-Knie wies sowohl im Flexionswinkel von $5^{\circ}$ als auch im Winkel von $90^{\circ}$ stabile und instabile Gleichgewichte auf insbesondere im Drehmoment. Hier zeigte sich, dass eine steigende Dominanz der Hamstring gegenüber dem Musculus gatrocnemius zu einem stabileren Gleichgewicht führte.

Die Ergebnisse zeigen, dass das Prothesendesign das Gleichgewicht beeinflusst. So konnten mit dem AEQUOS-Knie, welches der Gestaltung der natürlichen Gelenkflächen nachempfunden ist, durch Variationen des muskulären Kraftvektors stabile und instabile Gleichgewichtslagen erzeugt werden. 
$7 \quad$ Anhang

\begin{tabular}{|c|c|c|c|c|c|c|c|c|c|c|}
\hline \multirow{2}{*}{ 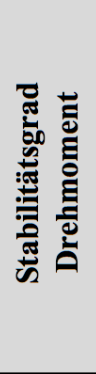 } & 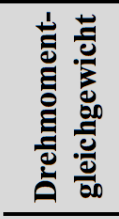 & 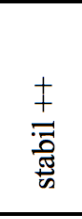 & 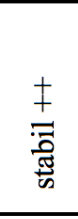 & 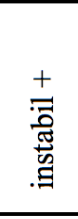 & 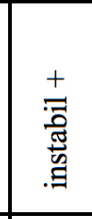 & 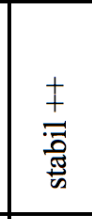 & 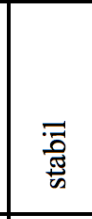 & 䔍 & 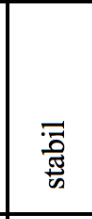 & 言 \\
\hline & 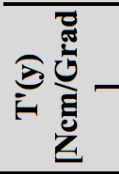 & 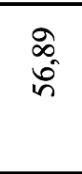 & $\begin{array}{l}q \\
f \\
g \\
g\end{array}$ & हुे & \begin{tabular}{l} 
n \\
\multirow{y}{y}{}
\end{tabular} & $\begin{array}{l}\text { ñ } \\
\text { bे }\end{array}$ & $\bar{m}_{\infty}$ & $\stackrel{\hat{N}}{\overrightarrow{\hat{N}}}$ & $\stackrel{m}{\cong}$ & 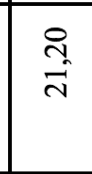 \\
\hline \multirow{2}{*}{ 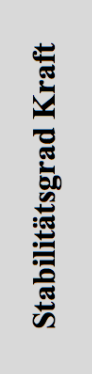 } & 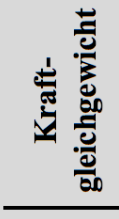 & $\begin{array}{l}\frac{7}{7} \\
\frac{7}{5} \\
\end{array}$ & 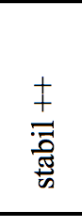 & 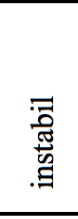 & 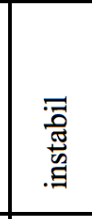 & 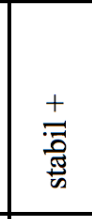 & 苞 & 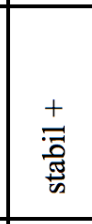 & $\begin{array}{l}\overline{7} \\
\text { 䭴 }\end{array}$ & 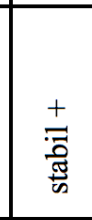 \\
\hline & 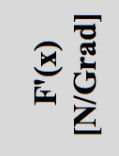 & : & 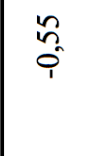 & $\stackrel{\infty}{\infty}$ & $\therefore$ & స్. & $\overrightarrow{\bar{i}}$ & $\begin{array}{l}\text { : } \\
\text { î. }\end{array}$ & $\begin{array}{l}\vec{i} \\
\stackrel{i}{0}\end{array}$ & 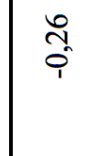 \\
\hline 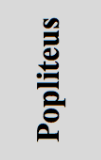 & $\underline{\mathbf{z}}$ & $\bar{i}$ & $\sigma^{2}$ & $\stackrel{\mathrm{I}}{\mathrm{I}}$ & $\therefore$ & $\therefore$ & $\tilde{\mathrm{d}}$ & $\begin{array}{l}\infty \\
\tilde{f} \\
\tilde{f}\end{array}$ & $\therefore$ & 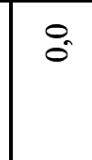 \\
\hline \multirow{2}{*}{ 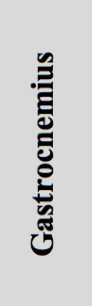 } & 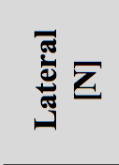 & 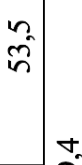 & $\left|\begin{array}{l}0 \\
\vdots \\
2\end{array}\right|$ & $\stackrel{0}{=}$ & $\stackrel{0}{=}$ & $\left|\begin{array}{c}\hat{f} \\
\mathcal{f}\end{array}\right|$ & $\mid \begin{array}{l}\ddot{\theta} \\
\dot{\theta}\end{array}$ & 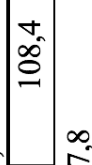 & : & 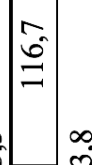 \\
\hline & 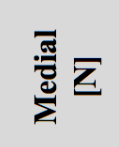 & 字 & $\mid \begin{array}{l}n \\
\overbrace{}^{-}\end{array}$ & 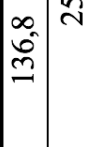 & \begin{tabular}{l}
2 \\
\hdashline \\
\hdashline
\end{tabular} & $\left|\begin{array}{c}\hat{f} \\
\hat{f}\end{array}\right|$ & $\left|\begin{array}{l}0 \\
\dot{f} \\
\dot{f}\end{array}\right|$ & 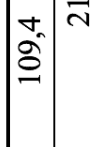 & $\left|\begin{array}{l}0 \\
: \\
\vdots \\
0\end{array}\right|$ & 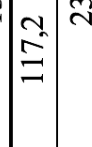 \\
\hline \multirow{2}{*}{ 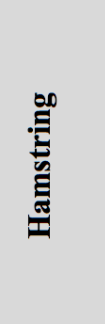 } & 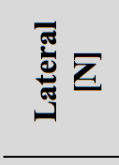 & $\hat{\tilde{\theta}}$ & $\mid \begin{array}{l}\vec{\infty} \\
\infty\end{array}$ & 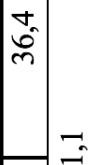 & 家 & 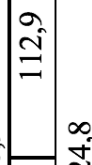 & $\left|\begin{array}{c}\mathcal{\alpha} \\
\mathcal{G}\end{array}\right|$ & 2 & $\hat{\imath}$ & $\left|\begin{array}{l}0 \\
\dot{a}\end{array}\right|$ \\
\hline & 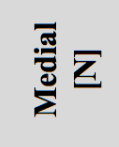 & 文 & $\left|\begin{array}{c}\infty \\
\infty \\
\infty \\
\infty\end{array}\right|$ & $\mid \begin{array}{l}\infty \\
\dot{m} \\
\dot{m}\end{array}$ & $\mid \vec{\partial}$ & $\stackrel{\theta}{\exists}$ & 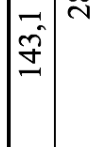 & के & $\left|\begin{array}{c}2 \\
\infty \\
\infty\end{array}\right|$ & $\begin{array}{l}\vec{\sigma} \\
\vec{\sigma}\end{array}$ \\
\hline 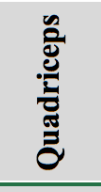 & $\underline{\underline{z}}$ & ণิ & $\stackrel{+}{\stackrel{5}{\Xi}}$ & $\begin{array}{l}\text { तू. } \\
\text { ర్స }\end{array}$ & aे & $\stackrel{\stackrel{m}{m}}{\vec{m}}$ & $\begin{array}{l}0 \\
\vec{n}\end{array}$ & 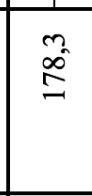 & $\tilde{m}^{n}$ & ๙ి. \\
\hline \multicolumn{2}{|c|}{ 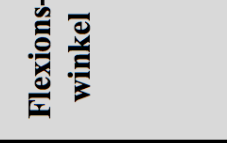 } & in & in & in & \&̊ & ஜి & in & in & : & 8 \\
\hline \multicolumn{2}{|l|}{ 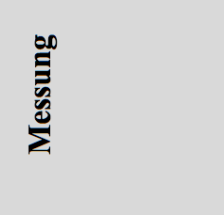 } & 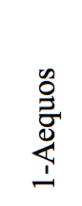 & 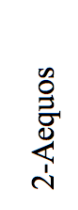 & 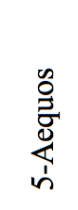 & 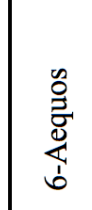 & 芯 & 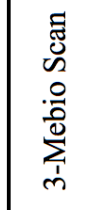 & 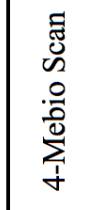 & 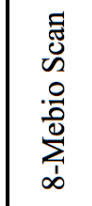 & 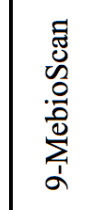 \\
\hline
\end{tabular}

Tabelle 10: Übersicht über die Muskelkräfte und Stabilitätsgrade. 


\begin{tabular}{|c|c|c|c|c|c|c|c|c|c|c|c|}
\hline \multirow{2}{*}{ 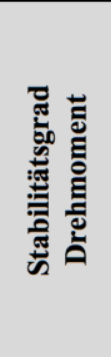 } & 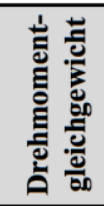 & 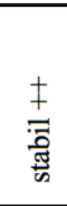 & 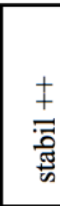 & & 焉 & 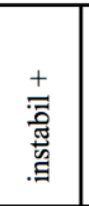 & 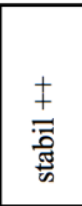 & $\begin{array}{l}\overline{\tilde{F}} \\
\text { 苟 }\end{array}$ & 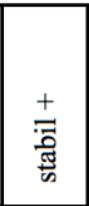 & 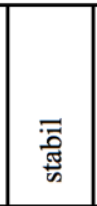 & $\frac{+}{\overrightarrow{7}}$ \\
\hline & 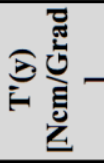 & $\begin{array}{l}\text { के } \\
\text { bी } \\
\tilde{n}^{2}\end{array}$ & $\begin{array}{l}\text { f } \\
\text { f }\end{array}$ & & $\begin{array}{l}\text { हे } \\
\text { के }\end{array}$ & 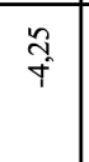 & 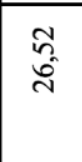 & $\overrightarrow{m_{0}}$ & $\begin{array}{l}\hat{n} \\
\vec{N}\end{array}$ & $\stackrel{m}{=}$ & 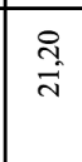 \\
\hline \multirow{2}{*}{ 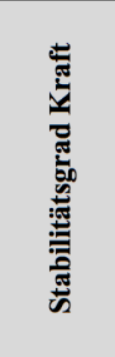 } & 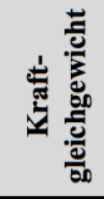 & 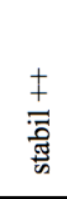 & 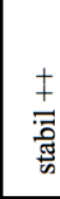 & & 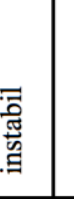 & 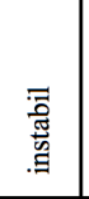 & 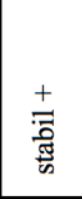 & 䔍 & 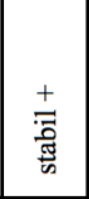 & 苞 & 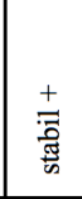 \\
\hline & 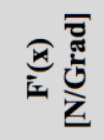 & ì & ñ & & $\stackrel{\infty}{\circ}$ & $\stackrel{n}{0}$ & స్ & $\begin{array}{l}= \\
0 \\
1\end{array}$ & $\begin{array}{l}\text { i̦ } \\
\text { i. }\end{array}$ & $\begin{array}{c}\vec{n} \\
\text { ô }\end{array}$ & $\begin{array}{l}\text { Nָ. } \\
\text { î. }\end{array}$ \\
\hline \multirow{2}{*}{ 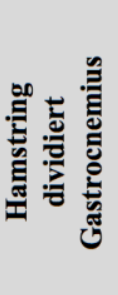 } & 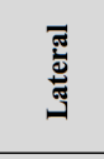 & i & $\mid \begin{array}{c}2 \\
0 \\
0\end{array}$ & $\underbrace{2}_{\infty}$ & $\stackrel{\infty}{\infty}$ & 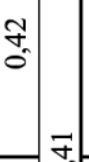 & \begin{tabular}{|}
0 \\
0 \\
0 \\
$i$
\end{tabular} \mid & $\begin{array}{l}\overrightarrow{0} \\
\dot{m}\end{array}$ & $\begin{array}{c}\infty \\
\infty \\
\infty\end{array}$ & $\stackrel{-}{-}$ & ${ }_{7}^{7}$ \\
\hline & 嵒 & 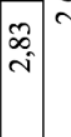 & $\begin{array}{l}2 \\
0 \\
0\end{array}$ & $0 \begin{array}{l}2 \\
2 \\
0 \\
0\end{array}$ & & & & $\begin{array}{l}n \\
\tilde{m} \\
m\end{array}$ & \begin{tabular}{|c|c|} 
& 0 \\
0 \\
0
\end{tabular} & & $\vec{F}_{0}$ \\
\hline 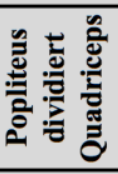 & & $\underset{\text { స్ }}{0}$ & of & & ${ }_{0}^{t}$ & : & 8 & $=$ & ๙̃ & 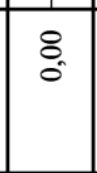 & 8 \\
\hline \multirow{2}{*}{ 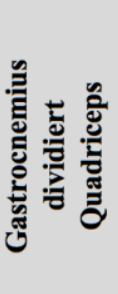 } & $\begin{array}{l}\text { बूँّ } \\
\text { जू }\end{array}$ & \begin{tabular}{|}
0 \\
\multirow{2}{*}{} \\
0 \\
0
\end{tabular} & $\mid \begin{array}{l}0 \\
\vdots \\
0 \\
0\end{array}$ & $\mid \begin{array}{l}q \\
q \\
0\end{array}$ & 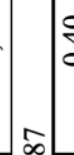 & $\begin{array}{l}q \\
0 \\
0\end{array}$ & $\frac{\Delta}{0}$ & స̃ & $\overrightarrow{0}$ & స్.ి & $\left.\right|_{\infty} ^{\substack{q \\
0}}$ \\
\hline & $\begin{array}{l}\text { 嵒 } \\
\frac{\pi}{2}\end{array}$ & స్. & $\begin{array}{l}\dot{2} \\
\vdots \\
0\end{array}$ & 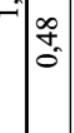 & & & & & & & \\
\hline \multirow{2}{*}{ 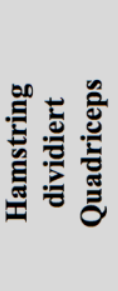 } & 苞 & 定 & $\mid \begin{array}{l}\infty \\
0 \\
0 \\
0\end{array}$ & $\stackrel{m}{m}$ & 5 & 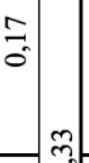 & $\begin{array}{l}0 \\
0 \\
0 \\
0\end{array}$ & $\left|\begin{array}{l}\hat{0} \\
0^{\circ}\end{array}\right|$ & 员 & $\overrightarrow{\tilde{o}}$ & $=$ \\
\hline & 嵒 & $\begin{array}{l}0 \\
0 \\
0 \\
0\end{array}$ & $\left|\begin{array}{l}\infty \\
\vdots \\
0 \\
0\end{array}\right|$ & 0 & & & $\mid$\begin{tabular}{l|l}
0 \\
0 \\
$0^{2}$ \\
0
\end{tabular} & & & & \\
\hline 这 & & in & in & & in & ஃू & ฉ̊ & in & in & ฉ̊ & ฉ̊ \\
\hline \multicolumn{2}{|l|}{ 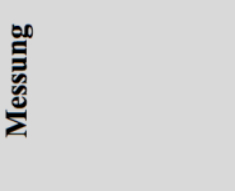 } & 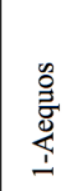 & 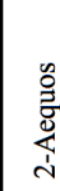 & & ni & 总 & 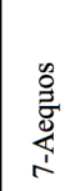 & 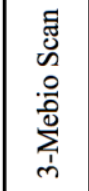 & 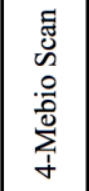 & 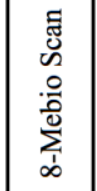 & $\begin{array}{l}\text { चี } \\
00 \\
000 \\
\sum_{0}^{0} \\
0\end{array}$ \\
\hline
\end{tabular}




\section{$8 \quad$ Literaturverzeichnis}

\subsection{Literatur}

Athanasiou K, Sanchez-Adams J: Engineering the Knee Meniscus (Synthesis Lectures on Tissue Engineering Series). Morgan and Claypool Publishers, San Rafael 2009 AWMF-Leitlinie Endoprothesen bei Gonarthrose (Register Nr. 012/008). AMWF online, 2011 https://www.awmf.org/leitlinien/detail/1l/012-008.html

Bizzini M: Sensomotorische Rehabilitation nach Beinverletzungen. Mit Fallbeispielen in allen Heilungsstadien. Georg Thieme, Stuttgart 2000

Blaha JD, Mancinelli CA, Simons WH, Kish VL, Thyagarajan G (2003): Kinematics of the human knee using an open chain cadaver model. Clin Orthop Relat Res. 410 , 25-34

Blümel A, Kraatz U: Beitrag zur funktionellen Anatomie und neuromuskulären Steuerung des Kniegelenks am Modelltier Schwein. Med. Diss. Greifswald 1997

Day B, Mackenzie WG, Shim SS, Leung G (1985): The vascular and nerve supply of the human meniscus. Arthroscopy $\underline{1}, 58-62$

Dyhre-Poulsen P, Krogsgaard MR (2000): Muscular reflexes elicited by electrical stimulation of the anterior cruciate ligament in humans. J Appl Physiol $\underline{89}$ (6), 21912195

Fanghänel J, Pera F, Anderhuber F, Nitsch R (Hrsg.): Fanghänel et al. A: Anatomie des Menschen. 17. Auflage; Walther de Gruyter, Berlin 2003

Fiedler C, Gezzi R, Frosch K-H, Wachowski M, Kubein-Meesenburg D, Dörner J, Fanghänel J, Nägerl H (2011): Mathematical study on the guidance of the tibiofemoral joint as theoretical background for total knee replacements. Acta Bioeng Biomech $\underline{13}$, $37-49$

Frosch KH, Floerkemeier T, Abicht C, Adam P, Dathe H, Fanghänel J, Stürmer KM, Kubein-Meesenburg D, Nägerl H (2009): A novel knee endoprosthesis with a physiological joint shape. Part 1: Biomechanical basics and tribological studies. Unfallchirurg $\underline{112}(2), 168-175$

Gerstenkamp G: Eine Methode zur Messung der Variabilität des Stabilitätsgrades von Knie-Endoprothesen. Med. Diss. Göttingen 2016 
Götz-Neumann K: Gehen verstehen - Ganganalyse in der Physiotherapie, 2. Auflage; Georg Thieme, Stuttgart 2006

Gray JC (1999): Neural and Vascular Anatomy of the Menisci of the Human Knee. J Orthop Sports Phys Ther 29 (1), 23-30

Halata Z, Haus J (1989): The ultrastructure of sensory nerve endings in human anterior cruciate ligament. Anal Embryol (Berl) 179 (5), 415-421

Hamai S, Moro-oka TA, Dunbar NJ, Miura H, Iwamoto Y, Banks SA (2013): In vivo healthy knee kinematics during dynamic full flexion. Biomed Res Int, 717546

Hill PF, Vedi V, Williams A, Iwaki H, Pinskerova V, Freeman MAR (2000): Tibiofemoral movement 2: The loaded and unloaded living knee studied by MRI. J Bone Joint Surg Br $\underline{82}$ (2), 1196-1198

Imhof H: Arthrose (Degenerative Gelenkerkrankungen). In: Stäbler A, Freyschmidt J (Hrsg.): Handbuch diagnostische Radiologie. Muskuloskelettales System 3. Springer, Berlin 2005, 314-356

Iwaki H, Pinskerova V, Freeman MA (2000): Tibiofemoral movement 1: The shapes and relative movements of the femur and tibia in the unloaded cadaver knee. J Bone Joint Surg Br $\underline{82}$ (8), 1189-1195

Jerosch J, Floren M (2000): Lebensqualitätsgewinn (SF-36) nach Implantation einer Knieendoprothese. Unfallchirurg 103, 371-374

Jerosch J, Heisel J: Knieendoprothetik. Springer, Berlin 1999

Johansson H (1991): Role of knee ligaments in proprioception and regulation of muscle stiffness. J Elektromyogr Kinesiol 1 (3), 158-179

Kapandji IA: Funktionelle Anatomie der Gelenke. Schematisierte und kommentierte Zeichnungen zur menschlichen Biomechanik. 5. Auflage; Georg Thieme, Stuttgart 2009

Katonis PG, Assimakopoulos AP, Agapitos MV, Exarchou EI (1991): Mechanoreceptors in the posterior cruciate ligament: Histologic study on cadaver knees. Acta Orthop Scand $\underline{62}$ (3), 276-278

Kirschner S (2011): Indikationskriterien und Epidemiologie des Gelenkersatzes. Zeitschrift für Evidenz, Fortbildung und Qualität im Gesundheitswesen 105 (2), 143 145 
Knösel M: Messung der isotonischen Stabilität von Tibiofemoralgelenk in der Hauptfunktion bei vorgegebener Flexion: Experimenteller Nachweis der Veränderbarkeit der Qualität des Gleichgewichtszustandes von labil zu stabil. Med. Diss. Göttingen 2003

Kubein-Meesenburg D, Nägerl H, Cortta H, Fanghänel J (1993): Biomechanische Prinzipien in Diarthrosen und Synarthrosen. Z. Orthop Unfall 131, 97-104

Mackeben S: Aufbau einer Apparatur zur Messung der mechanischen Stabilität des menschlichen Tibiofemoralgelenks. Phy. Diplomarbeit Göttingen 1997

Mansour M, Spiering S, Lee C, Dathe H, Kalscheuer AK, Kubein-Meesenburg D, Nägerl H (2004): Evidence for IHA migration during axial rotation of a lumbar spine segment by using a novel high-resolution 6D kinematic tracking system. J Biomech $\underline{37}(4), 583-592$

Markolf KL, Jackson SR, Foster B, McAllister DR (2014): ACL forces and knee kinematics produced by axial tibial compression during a passive flexion-extension cycle. J Orthop Res $\underline{32}$ (1), 89-95

Martelli S, Pinskerova V (2002): The shapes of the tibial and femoral articular surfaces in relation to tibiofemoral movement. J Bone Joint Surg Br $\underline{84}$ (4), 607-613

Menschik A (1974): Mechanik des Kniegelenks. Z Orthop 112, 481-495

Meyer GH: Die Statik und Mechanik des menschlichen Knochengerüstes. Wilhelm Engelmann, Leipzig 1873

Mitchell FL Jr., Galen Mitchell PK: Handbuch der Muskelenergietechniken. Band 3: Diagnostik und Therapie: Becken und Sakrum. Hippokrates Verlag, Stuttgart 2005 Mow VC, Gu WY, Chen FH: Structure and function of articular cartilage and meniscus. In: Mow VC, Huiskes R (Hrsg.): Basic Orthopedic Biomechanics \& Mechanobiology. 3. Auflage; Lippincott Williams \& Wilkins, Philadelphia 2005, 181258

Nägerl H: Biomechanische Prinzipien in Diarthrosen und Synarthrosen. Med. Habilitationsschrift Göttingen 1990

Nägerl H, Kubein-Meesenburg D, Cotta H, Fanghänel J (1993): Biomechanische Prinzipien in Diarthrosen und Synarthrosen. III. Mechanik des Tibiofemoralgelenks und Rolle der Kreuzbänder. Z Orthop 131, 385-396 
Nägerl H, Blümel A, Kraatz U, Kubein-Meesenburg D, Miehe B, Fanghänel J (2002): The sensory apparatus for perception in the tibiofemoral joint and outlines of a functional knee endoprosthesis. Acta Bioeng Biomech $\underline{4}$ (1), 319-320

Nägerl H, Abicht C, Adam P, Dathe H, Fanghänel J, Thomsen M, Döhler JR, KubeinMeesenburg D (2004): A novel total knee replacement with nearly natural joint geometry. Part I: Biomechanical conception and design of the articulating surfaces, Ann Anat $\underline{186}$ (Supplement), 33

Nägerl H, Frosch KH, Wachowski MM, Dumont C, Abicht CH, Adam P, KubeinMeesenburg D (2008): A novel total knee replacement by rolling articulating surfaces. In vivo functional measurements and tests. Acta Bioeng Biomech $\underline{10}$ (1), 55-60

Perry J: Ganganalyse - Norm und Pathologie des Gehens. Urban \& Fischer, München 2003

Planert J, Modler H, Lüdecke K, Eger M (1992): A miniaturised force-torque sensor with six degrees of freedom for dental measurements. Clin Phys Physiol Meas $\underline{13}$ (3), $241-248$

Prometheus - Allgemeine Anatomie und Bewegungssystem. Hrsg. v. Schünke M, Schulte E, Schumacher U, 4. Aufl.; Georg Thieme, Stuttgart 2014

Saygi B, Yildirim Y, Berker N, Ofluoglu D, Karadag-Saygi E, Karahan M (2005): Evaluation of neurosensory function of the medial meniscus in humans. Arthroscopy 21 (12), 1468-1472

Schünke M: Funktionelle Anatomie - Topografie und Funktion des Bewegungssystems. Georg Thieme, Stuttgart 2000

Schultz RA, Miller DC, Kerr CS, Micheli L (1984): Mechanoreceptors in human cruciate ligament. A biological study. J Bone Joint Surg Am 66 (7), 1072-1075

Schutte MJ, Dabizies EJ, Zimny ML, Happel LT (1987): Neural anatomy of the human anterior cruciate ligament. J Bone Joint Surg Am $\underline{69}$ (2), 243

Solomonow M, Baratta R, Zhou BH, Shoji H, Bose W, Beck C, D'Ambrosia R. (1987): The synergistic action of the anterior cruciate ligament and thigh muscles in maintaining joint stability. Am J Sports Med 15 (3), 207-213

Strasser H: Lehrbuch der Muskel- und Gelenkmechanik. III. Band: Die untere Extremität. Springer, Berlin 1917 
Stühmer C: Konzeptionierung einer Meßmethode zur quantitativen Erfassung der isotonischen Stabilität des Tibiofemoralgelenks des Hausschweins in der Hauptfunktionsebene. Med. Diss. Göttingen 2002

Thomsen M, Mannel H, Spiering S, Dathe H, Kubein-Meesenburg D, Nägerl H (2002): Zur Biomechanik des Tibiofemoralgelenks und deren Umsetzung bei Knieorthesen. Orthopade 31, 914-920

Tilmann B (Hrsg.): Rauber /Kopsch - Anatomie des Menschen. Band I, Bewegungsapparat. 3. Auflage; Georg Thieme, Stuttgart 2003

van den Bogert AJ, Reinschmidt C, Lundberg A (2008): Helical axes of skeletal knee joint motion during running. J Biomech $\underline{41}$ (8): 1632-1638

Weber W, Weber E: Die Mechanik der menschlichen Gehwerkzeuge. Dietrichsche Buchhandlung, Göttingen 1836

Wetz HH, Jacob HAC (2001): Funktionelle Anatomie und Kinematik des Femurotibialgelenks. Forschungsergebnisse von 1836 bis 1950. Orthopade $\underline{30}$ (3), $135-144$

Witzel U: Biomechanische und tribiologische Aspekte der Kniegelenkendoprothetik. In: Eulert J, Hassenpflug J (Hrsg.): Praxis der Knieendoprothetik. Springer, Berlin 2000

Zimny ML, Albright DJ, Dabezies E (1988): Mechanoreceptors in the human medial meniscus. Acta Anat (Basel) 133 (1), 35-40

\subsection{Internetquellen}

http://www.aap.de/de/Produkte/Endoprothetik/Knieendoprothetik/Mebio_Knie/index .html; Zugriff am 04.02.2005

http://www.aequos.de/de/arzt/reproduktion.htm; Zugriff am 11.04.2018

http://my.me.queensu.ca/People/Deluzio/DataAnalysis.html; Zugriff am 11.04.2018

http://www.neu.uni-

bayreuth.de/de/Uni_Bayreuth/Fakultaeten/6_FAN/Metallische_Werkstoffe_neu_unfe rtig/de/research/polymer/index.html; Zugriff am 09.04.2018 


\section{Danksagung}

Mein besonderer Dank gilt Herrn Prof. Dr. Hans Nägerl für die Bereitstellung des Themas und die gute Betreuung sowie die fruchtbaren fachlichen Gespräche und die Vermittlung seiner physikalischen Betrachtung der Medizin.

Ebenso danke ich Prof. Kubein-Meesenburg für die Unterstützung und die fachlich anregenden Diskussionen im Doktoranden-Seminar.

Für die Unterstützung bei der Umsetzung des Themas möchte ich mich bei Herrn Dr. rer. Nat. Dipl.-Phys. Henning Dathe besonders bedanken.

Ebenfalls danke ich Dr. med. dent. Gustav-Ludwig Gerstenkamp für die fachlichen Diskussionen.

Schließlich danke ich Frau Maxi Katharina Albrecht, M.A., für die kritischen Diskussionen und Anregungen. 
Lebenslauf 